Table 1. SHRIMP U-Pb data for zircon grains from sample BATAL

\begin{tabular}{|c|c|c|c|c|c|c|c|c|c|c|c|c|c|c|c|c|c|c|c|c|c|c|}
\hline \multirow[b]{2}{*}{$\begin{array}{c}\text { Grain. } \\
\text { spot }\end{array}$} & \multirow[b]{2}{*}{$\underset{(\mathrm{ppm})}{\mathrm{U}}$} & \multirow[b]{2}{*}{$\begin{array}{c}\text { Th } \\
\text { (ppm) }\end{array}$} & & & & & & Total R & atios & & & & adiogeni & Ratios & & & & & & e (Ma) & & \\
\hline & & & $\mathrm{Th} / \mathrm{U}$ & $\begin{array}{l}{ }^{206} \mathrm{~Pb}^{\star} \\
(\mathrm{ppm})\end{array}$ & $\begin{array}{l}{ }^{204} \mathrm{~Pb} / \\
{ }^{206} \mathrm{~Pb}\end{array}$ & $\begin{array}{l}\mathrm{f}_{206} \\
\%\end{array}$ & $\begin{array}{l}{ }^{238} \mathrm{U} / \\
{ }^{206} \mathrm{~Pb}\end{array}$ & \pm & $\begin{array}{l}{ }^{207} \mathrm{~Pb} / \\
{ }^{006} \mathrm{~Pb}\end{array}$ & \pm & $\begin{array}{l}{ }^{206} \mathrm{~Pb} / \\
{ }^{233} \mathrm{U}\end{array}$ & \pm & $\begin{array}{c}{ }^{207} \mathrm{~Pb} / \\
{ }^{235} \mathrm{U}\end{array}$ & \pm & ${ }^{207} \mathrm{~Pb} /$ & \pm & $\rho$ & $\begin{array}{c}{ }^{206} \mathrm{~Pb} / \\
{ }^{238} \mathrm{U}\end{array}$ & \pm & $\begin{array}{l}{ }^{207} \mathrm{~Pb} / \\
{ }^{206} \mathrm{~Pb}\end{array}$ & \pm & $\begin{array}{c}\% \\
\text { Disc }\end{array}$ \\
\hline 1.1 & 146 & 83 & 0.56 & 13 & 0.000127 & 0.38 & 9.767 & 0.142 & 0.0638 & 0.0017 & 0.1020 & 0.0015 & & & & & & 626 & 9 & & & \\
\hline 2.1 & 142 & 82 & 0.58 & 19 & 0.000235 & 0.40 & 6.338 & 0.089 & 0.0732 & 0.0014 & 0.1571 & 0.0022 & 1.512 & 0.055 & 0.0698 & 0.0024 & 0.389 & 941 & 12 & 923 & 69 & -2 \\
\hline 3.1 & 396 & 219 & 0.55 & 57 & 0.000005 & 0.01 & 5.955 & 0.069 & 0.0716 & 0.0006 & 0.1680 & 0.0021 & 1.665 & 0.052 & 0.0719 & 0.0016 & 0.820 & 1001 & 12 & 983 & 44 & -2 \\
\hline 4.1 & 154 & 58 & 0.38 & 21 & - & $<0.01$ & 6.375 & 0.086 & 0.0722 & 0.0011 & 0.1569 & 0.0021 & 1.570 & 0.032 & 0.0726 & 0.0011 & 0.656 & 940 & 12 & 1001 & 32 & 6 \\
\hline 5.1 & 1918 & 136 & 0.07 & 848 & 0.000005 & 0.01 & 1.943 & 0.020 & 0.1695 & 0.0006 & 0.5147 & 0.0053 & 12.023 & 0.130 & 0.1694 & 0.0006 & 0.953 & 2677 & 23 & 2552 & 5 & -5 \\
\hline 6.1 & 276 & 128 & 0.46 & 38 & 0.000180 & 0.31 & 6.170 & 0.075 & 0.0745 & 0.0008 & 0.1616 & 0.0020 & 1.603 & 0.035 & 0.0720 & 0.0013 & 0.553 & 966 & 11 & 985 & 37 & 2 \\
\hline 7.1 & 469 & 191 & 0.41 & 100 & 0.000013 & 0.02 & 4.025 & 0.045 & 0.0892 & 0.0005 & 0.2485 & 0.0029 & 3.060 & 0.064 & 0.0893 & 0.0011 & 0.876 & 1431 & 15 & 1411 & 23 & -1 \\
\hline 8.1 & 645 & 257 & 0.40 & 265 & 0.000049 & 0.07 & 2.090 & 0.022 & 0.1713 & 0.0005 & 0.4781 & 0.0051 & 11.251 & 0.125 & 0.1707 & 0.0005 & 0.963 & 2519 & 22 & 2564 & 5 & 2 \\
\hline 9.1 & 70 & 80 & 1.15 & 25 & - & $<0.01$ & 2.362 & 0.036 & 0.1615 & 0.0018 & 0.4237 & 0.0065 & 9.469 & 0.182 & 0.1621 & 0.0019 & 0.798 & 2277 & 29 & 2478 & 19 & 8 \\
\hline 10.1 & 362 & 546 & 1.51 & 49 & - & $<0.01$ & 6.363 & 0.074 & 0.0714 & 0.0007 & 0.1572 & 0.0018 & 1.551 & 0.023 & 0.0716 & 0.0007 & 0.772 & 941 & 10 & 974 & 19 & 3 \\
\hline 11.1 & 149 & 104 & 0.69 & 24 & 0.000224 & 0.38 & 5.288 & 0.072 & 0.0820 & 0.0011 & 0.1884 & 0.0026 & 2.047 & 0.054 & 0.0788 & 0.0018 & 0.517 & 1113 & 14 & 1167 & 45 & 5 \\
\hline 12.1 & 499 & 199 & 0.40 & 129 & 0.000026 & 0.04 & 3.331 & 0.037 & 0.1007 & 0.0005 & 0.3001 & 0.0033 & 4.151 & 0.051 & 0.1003 & 0.0005 & 0.899 & 1692 & 16 & 1630 & 10 & -4 \\
\hline 13.1 & 1146 & 65 & 0.06 & 177 & 0.000410 & 0.67 & 5.555 & 0.058 & 0.0967 & 0.0004 & 0.1788 & 0.0019 & 2.244 & 0.028 & 0.0910 & 0.0006 & 0.825 & 1061 & 10 & 1447 & 14 & 27 \\
\hline 14.1 & 490 & 167 & 0.34 & 97 & 0.000020 & 0.03 & 4.337 & 0.048 & 0.0923 & 0.0006 & 0.2305 & 0.0026 & 2.926 & 0.038 & 0.0921 & 0.0006 & 0.857 & 1337 & 13 & 1469 & 13 & 9 \\
\hline 15.1 & 918 & 447 & 0.49 & 150 & & $<0.01$ & 5.261 & 0.056 & 0.0784 & 0.0004 & 0.1901 & 0.0020 & 2.056 & 0.024 & 0.0785 & 0.0004 & 0.901 & 1122 & 11 & 1159 & 10 & 3 \\
\hline 16.1 & 249 & 271 & 1.09 & 34 & 0.000095 & 0.16 & 6.222 & 0.076 & 0.0719 & 0.0008 & 0.1604 & 0.0020 & 1.562 & 0.034 & 0.0706 & 0.0012 & 0.569 & 959 & 11 & 946 & 36 & -1 \\
\hline 17.1 & 208 & 118 & 0.57 & 45 & - & $<0.01$ & 3.977 & 0.049 & 0.0930 & 0.0008 & 0.2516 & 0.0031 & 3.242 & 0.049 & 0.0935 & 0.0008 & 0.809 & 1446 & 16 & 1497 & 17 & 3 \\
\hline 18.2 & 593 & 422 & 0.71 & 33 & 0.000120 & 0.49 & 15.581 & 0.174 & 0.0586 & 0.0007 & 0.0639 & 0.0007 & & & & & & 399 & 4 & & & \\
\hline 19.1 & 1057 & 544 & 0.51 & 488 & 0.000009 & 0.01 & 1.861 & 0.019 & 0.1917 & 0.0004 & 0.5387 & 0.0060 & 14.414 & 0.233 & 0.1941 & 0.0015 & 0.899 & 2778 & 25 & 2777 & 13 & 0 \\
\hline 20.1 & 195 & 121 & 0.62 & 30 & 0.000113 & 0.19 & 5.614 & 0.070 & 0.0748 & 0.0009 & 0.1787 & 0.0025 & 1.904 & 0.067 & 0.0773 & 0.0020 & 0.803 & 1060 & 13 & 1129 & 51 & 6 \\
\hline 21.1 & 99 & 57 & 0.58 & 41 & 0.000150 & 0.21 & 2.083 & 0.029 & 0.1710 & 0.0012 & 0.4790 & 0.0066 & 11.171 & 0.179 & 0.1692 & 0.0014 & 0.864 & 2523 & 29 & 2549 & 14 & 1 \\
\hline 22.1 & 3092 & 1654 & 0.53 & 389 & 0.000004 & 0.01 & 6.827 & 0.070 & 0.0693 & 0.0002 & 0.1465 & 0.0015 & 1.399 & 0.015 & 0.0692 & 0.0002 & 0.944 & 881 & 8 & 906 & 7 & 3 \\
\hline 23.1 & 55 & 101 & 1.83 & 20 & 0.000189 & 0.27 & 2.374 & 0.039 & 0.1601 & 0.0016 & 0.4202 & 0.0069 & 9.138 & 0.189 & 0.1577 & 0.0020 & 0.791 & 2261 & 31 & 2432 & 22 & 7 \\
\hline 24.1 & 1070 & 617 & 0.58 & 419 & - & $<0.01$ & 2.194 & 0.023 & 0.1613 & 0.0020 & 0.4557 & 0.0048 & 10.134 & 0.165 & 0.1613 & 0.0020 & 0.641 & 2421 & 21 & 2469 & 21 & 2 \\
\hline 25.1 & 683 & 1509 & 2.21 & 57 & 0.000112 & 0.20 & 10.327 & 0.114 & 0.0596 & 0.0005 & 0.0966 & 0.0011 & & & & & & 595 & 6 & & & \\
\hline 26.2 & 1406 & 107 & 0.08 & 195 & 0.000006 & 0.01 & 6.205 & 0.064 & 0.0700 & 0.0003 & 0.1612 & 0.0017 & 1.558 & 0.020 & 0.0701 & 0.0004 & 0.916 & 963 & 9 & 932 & 11 & -3 \\
\hline 27.1 & 326 & 224 & 0.69 & 47 & 0.000046 & 0.08 & 5.896 & 0.068 & 0.0723 & 0.0006 & 0.1700 & 0.0022 & 1.744 & 0.060 & 0.0744 & 0.0018 & 0.831 & 1012 & 12 & 1052 & 50 & 4 \\
\hline 28.1 & 301 & 245 & 0.81 & 50 & 0.000111 & 0.19 & 5.168 & 0.059 & 0.0803 & 0.0007 & 0.1931 & 0.0022 & 2.096 & 0.032 & 0.0787 & 0.0008 & 0.744 & 1138 & 12 & 1165 & 20 & 2 \\
\hline 29.1 & 308 & 98 & 0.32 & 41 & 0.000082 & 0.14 & 6.394 & 0.074 & 0.0727 & 0.0009 & 0.1562 & 0.0018 & 1.540 & 0.029 & 0.0715 & 0.0011 & 0.612 & 936 & 10 & 973 & 31 & 4 \\
\hline 30.1 & 971 & 364 & 0.38 & 134 & 0.000018 & 0.03 & 6.226 & 0.066 & 0.0707 & 0.0004 & 0.1608 & 0.0018 & 1.594 & 0.033 & 0.0719 & 0.0009 & 0.872 & 961 & 10 & 982 & 26 & 2 \\
\hline 31.1 & 68 & 29 & 0.43 & 11 & 0.000218 & 0.37 & 5.359 & 0.084 & 0.0765 & 0.0014 & 0.1863 & 0.0031 & 1.925 & 0.081 & 0.0750 & 0.0024 & 0.730 & 1101 & 17 & 1067 & 65 & -3 \\
\hline 32.1 & 1627 & 54 & 0.03 & 141 & 0.000016 & 0.03 & 9.902 & 0.103 & 0.0607 & 0.0003 & 0.1010 & 0.0011 & & & & & & 620 & 6 & & & \\
\hline 33.1 & 2545 & 62 & 0.02 & 350 & 0.000006 & 0.01 & 6.240 & 0.064 & 0.0712 & 0.0004 & 0.1603 & 0.0016 & 1.572 & 0.019 & 0.0711 & 0.0004 & 0.863 & 958 & 9 & 962 & 12 & 0 \\
\hline 34.1 & 275 & 109 & 0.39 & 59 & - & $<0.01$ & 4.004 & 0.046 & 0.0903 & 0.0006 & 0.2498 & 0.0029 & 3.110 & 0.041 & 0.0903 & 0.0006 & 0.858 & 1437 & 15 & 1432 & 13 & 0 \\
\hline 35.1 & 768 & 83 & 0.11 & 401 & - & $<0.01$ & 1.643 & 0.017 & 0.2472 & 0.0010 & 0.6085 & 0.0064 & 20.744 & 0.234 & 0.2472 & 0.0010 & 0.931 & 3064 & 26 & 3167 & 7 & 3 \\
\hline 36.1 & 601 & 432 & 0.72 & 47 & 0.000068 & 0.06 & 10.885 & 0.121 & 0.0595 & 0.0007 & 0.0918 & 0.0010 & & & & & & 566 & 6 & & & \\
\hline 36.2 & 1050 & 656 & 0.62 & 68 & 0.000103 & 0.32 & 13.214 & 0.141 & 0.0591 & 0.0005 & 0.0754 & 0.0008 & & & & & & 469 & 5 & & & \\
\hline 37.1 & 146 & 34 & 0.23 & 72 & 0.000030 & 0.04 & 1.737 & 0.023 & 0.1701 & 0.0011 & 0.5753 & 0.0077 & 13.462 & 0.205 & 0.1697 & 0.0012 & 0.884 & 2930 & 32 & 2555 & 12 & -15 \\
\hline 38.1 & 133 & 162 & 1.22 & 32 & 0.000076 & 0.12 & 3.631 & 0.046 & 0.0991 & 0.0009 & 0.2751 & 0.0035 & 3.719 & 0.064 & 0.0980 & 0.0011 & 0.737 & 1567 & 18 & 1587 & 22 & 1 \\
\hline 39.1 & 308 & 197 & 0.64 & 85 & 0.000034 & 0.05 & 3.118 & 0.035 & 0.1155 & 0.0006 & 0.3205 & 0.0036 & 5.084 & 0.065 & 0.1150 & 0.0007 & 0.884 & 1792 & 18 & 1880 & 11 & 5 \\
\hline 40.1 & 488 & 69 & 0.14 & 51 & 0.000035 & 0.13 & 8.219 & 0.090 & 0.0650 & 0.0005 & 0.1215 & 0.0014 & & & & & & 739 & 8 & & & \\
\hline 41.1 & 689 & 306 & 0.44 & 59 & 0.000011 & 0.23 & 9.986 & 0.108 & 0.0622 & 0.0005 & 0.0999 & 0.0011 & & & & & & 614 & 6 & & & \\
\hline 42.1 & 336 & 107 & 0.32 & 55 & 0.000028 & 0.05 & 5.253 & 0.059 & 0.0812 & 0.0006 & 0.1903 & 0.0021 & 2.120 & 0.030 & 0.0808 & 0.0007 & 0.795 & 1123 & 12 & 1217 & 17 & 8 \\
\hline 43.1 & 1269 & 432 & 0.34 & 115 & 0.000053 & $<0.01$ & 9.477 & 0.099 & 0.0611 & 0.0003 & 0.1055 & 0.0011 & & & & & & 647 & 7 & & & \\
\hline 44.1 & 150 & 226 & 1.51 & 21 & - & $<0.01$ & 6.061 & 0.078 & 0.0717 & 0.0009 & 0.1651 & 0.0021 & 1.639 & 0.030 & 0.0720 & 0.0009 & 0.704 & 985 & 12 & 987 & 27 & 0 \\
\hline 45.1 & 282 & 86 & 0.30 & 50 & 0.000131 & 0.21 & 4.828 & 0.055 & 0.0897 & 0.0008 & 0.2067 & 0.0024 & 2.504 & 0.040 & 0.0879 & 0.0010 & 0.715 & 1211 & 13 & 1380 & 22 & 12 \\
\hline 46.1 & 403 & 650 & 1.61 & 160 & 0.000104 & 0.15 & 2.163 & 0.024 & 0.1626 & 0.0005 & 0.4616 & 0.0050 & 10.265 & 0.118 & 0.1613 & 0.0006 & 0.948 & 2447 & 22 & 2469 & 6 & 1 \\
\hline 47.1 & 738 & 72 & 0.10 & 90 & 0.000036 & 0.06 & 7.039 & 0.075 & 0.0681 & 0.0004 & 0.1420 & 0.0015 & 1.323 & 0.017 & 0.0676 & 0.0005 & 0.811 & 856 & 9 & 856 & 16 & 0 \\
\hline 48.1 & 110 & 121 & 1.10 & 15 & & $<0.01$ & 6.310 & 0.086 & 0.0704 & 0.0010 & 0.1588 & 0.0022 & 1.580 & 0.041 & 0.0721 & 0.0016 & 0.522 & 950 & 12 & 990 & 45 & 4 \\
\hline 49.1 & 455 & 308 & 0.68 & 52 & 0.000026 & 0.04 & 7.470 & 0.081 & 0.0649 & 0.0005 & 0.1338 & 0.0015 & 1.191 & 0.016 & 0.0645 & 0.0005 & 0.794 & 810 & 8 & 759 & 18 & -7 \\
\hline 50.1 & 1450 & 612 & 0.42 & 198 & 0.000030 & 0.05 & 6.284 & 0.065 & 0.0766 & 0.0003 & 0.1591 & 0.0016 & 1.671 & 0.019 & 0.0762 & 0.0003 & 0.928 & 952 & 9 & 1100 & 8 & 13 \\
\hline 51.1 & 35 & 43 & 1.20 & 13 & 0.000113 & 0.16 & 2.416 & 0.041 & 0.1585 & 0.0032 & 0.4133 & 0.0071 & 8.952 & 0.251 & 0.1571 & 0.0035 & 0.610 & 2230 & 32 & 2425 & 38 & 8 \\
\hline 52.1 & 243 & 161 & 0.66 & 45 & 0.000021 & 0.03 & 4.689 & 0.054 & 0.0788 & 0.0006 & 0.2136 & 0.0027 & 2.358 & 0.073 & 0.0801 & 0.0017 & 0.844 & 1248 & 14 & 1199 & 42 & -4 \\
\hline 53.1 & 335 & 228 & 0.68 & 47 & - & $<0.01$ & 6.068 & 0.068 & 0.0707 & 0.0006 & 0.1649 & 0.0018 & 1.618 & 0.023 & 0.0712 & 0.0006 & 0.794 & 984 & 10 & 963 & 17 & -2 \\
\hline 54.1 & 355 & 294 & 0.83 & 44 & 0.000015 & 0.03 & 6.890 & 0.077 & 0.0703 & 0.0006 & 0.1451 & 0.0016 & 1.403 & 0.020 & 0.0701 & 0.0006 & 0.786 & 873 & 9 & 932 & 18 & 6 \\
\hline 55.1 & 1007 & 78 & 0.08 & 186 & - & $<0.01$ & 4.653 & 0.049 & 0.0813 & 0.0007 & 0.2149 & 0.0022 & 2.410 & 0.033 & 0.0813 & 0.0007 & 0.760 & 1255 & 12 & 1229 & 18 & -2 \\
\hline 56.1 & 1706 & 142 & 0.08 & 410 & 0.000003 & 0.01 & 3.575 & 0.037 & 0.0932 & 0.0007 & 0.2797 & 0.0029 & 3.592 & 0.045 & 0.0931 & 0.0007 & 0.825 & 1590 & 14 & 1491 & 13 & -7 \\
\hline 57.1 & 2479 & 477 & 0.19 & 405 & 0.000005 & 0.01 & 5.260 & 0.054 & 0.0742 & 0.0002 & 0.1903 & 0.0020 & 1.968 & 0.028 & 0.0750 & 0.0004 & 0.949 & 1123 & 11 & 1069 & 11 & -5 \\
\hline 58.1 & 948 & 121 & 0.13 & 388 & 0.000009 & 0.01 & 2.097 & 0.022 & 0.1670 & 0.0005 & 0.4769 & 0.0049 & 10.974 & 0.118 & 0.1669 & 0.0005 & 0.962 & 2514 & 22 & 2527 & 5 & 1 \\
\hline 59.1 & 155 & 257 & 1.65 & 10 & 000381 & 1.25 & 13.766 & 0.183 & 0.0660 & 0.0029 & 0.0717 & 0.0010 & & & & & & 447 & 6 & & & \\
\hline 59.2 & 187 & 241 & 1.29 & 14 & 0.000256 & 0.08 & 11.803 & 0.154 & 0.0585 & 0.0010 & 0.0847 & 0.0011 & & & & & & 524 & 7 & & & \\
\hline 60.1 & 400 & 185 & 0.46 & 33 & 0.000007 & 0.01 & 10.382 & 0.116 & 0.0604 & 0.0006 & 0.0963 & 0.0011 & & & & & & 593 & 6 & & & \\
\hline 61.1 & 176 & 131 & 0.74 & 23 & - & $<0.01$ & 6.530 & 0.080 & 0.0707 & 0.0009 & 0.1532 & 0.0019 & 1.500 & 0.027 & 0.0710 & 0.0009 & 0.682 & 919 & 10 & 958 & 27 & 4 \\
\hline 62.1 & 305 & 240 & 0.79 & 41 & 0.000018 & 0.03 & 6.450 & 0.073 & 0.0719 & 0.0006 & 0.1550 & 0.0018 & 1.532 & 0.023 & 0.0717 & 0.0007 & 0.765 & 929 & 10 & 977 & 19 & 5 \\
\hline 63.1 & 1771 & 162 & 0.09 & 255 & 0.000021 & 0.04 & 5.969 & 0.061 & 0.0715 & 0.0002 & 0.1675 & 0.0017 & 1.652 & 0.021 & 0.0715 & 0.0003 & 0.945 & 999 & 10 & 972 & 9 & -3 \\
\hline 64.1 & 907 & 355 & 0.39 & 125 & 0.000005 & 0.01 & 6.216 & 0.065 & 0.0708 & 0.0004 & 0.1610 & 0.0018 & 1.585 & 0.033 & 0.0714 & 0.0009 & 0.883 & 962 & 10 & 969 & 25 & 1 \\
\hline 65.1 & 20 & 7 & 0.37 & 4 & 0.000479 & 0.80 & 4.404 & 0.101 & 0.0857 & 0.0023 & 0.2268 & 0.0055 & 2.652 & 0.145 & 0.0848 & 0.0034 & 0.722 & 1318 & 29 & 1311 & 79 & -1 \\
\hline
\end{tabular}

Notes : $\quad 1$. Uncertainties given at the one $\sigma$ level.

2. Error in Temora reference zircon calibration was $0.71 \%$ for the analytical session. ( not included in above errors but required when comparing ${ }^{206} \mathrm{~Pb} /{ }^{338} \mathrm{U}$ data from different mounts).

3. $\mathrm{f}_{206} \%$ denotes the percentage of ${ }^{206} \mathrm{~Pb}$ that is common $\mathrm{Pb}$.

4. For areas older than $\sim 800 \mathrm{Ma}$ correction for common $\mathrm{Pb}$ made using the measured ${ }^{204} \mathrm{~Pb}{ }^{206} \mathrm{~Pb}$ ratio.

5. For areas younger than $\sim 800 \mathrm{Ma}$ correction for common $\mathrm{Pb}$ made using the measured ${ }^{238} \mathrm{U} /{ }^{206} \mathrm{~Pb}$ and ${ }^{207} \mathrm{~Pb} / 206 \mathrm{~Pb}$ ratios following Tera and Wasserburg (1972) as outlined in Williams (1998).

6. For $\%$ Disc, $0 \%$ denotes a concordant analysis. 
Table 2. SHRIMP U-Pb data for zircon grains from sample MS-2

\begin{tabular}{|c|c|c|c|c|c|c|c|c|c|c|c|c|c|c|c|c|c|c|c|c|c|c|}
\hline \multirow[b]{2}{*}{$\begin{array}{c}\text { Grain. } \\
\text { spot }\end{array}$} & \multirow[b]{2}{*}{$\begin{array}{c}U \\
(\mathrm{ppm})\end{array}$} & \multirow[b]{2}{*}{$\begin{array}{c}\text { Th } \\
(\mathrm{ppm})\end{array}$} & \multirow[b]{2}{*}{$\mathrm{Th} / \mathrm{U}$} & & & & & Total F & atios & & & & adiogeni & Ratios & & & & & & $\mathrm{e}(\mathrm{Ma})$ & & \\
\hline & & & & $\begin{array}{l}{ }^{206} \mathrm{~Pb}^{*} \\
(\mathrm{ppm})\end{array}$ & $\begin{array}{l}{ }^{204} \mathrm{~Pb} / \\
{ }^{206} \mathrm{~Pb}\end{array}$ & $\begin{array}{c}\mathrm{f}_{206} \\
\%\end{array}$ & $\begin{array}{l}{ }^{238} \mathrm{U} / \\
{ }^{206} \mathrm{~Pb}\end{array}$ & \pm & $\begin{array}{l}{ }^{207} \mathrm{~Pb} / \\
{ }^{206} \mathrm{~Pb}\end{array}$ & \pm & $\begin{array}{l}{ }^{206} \mathrm{~Pb} / \\
{ }^{238} \mathrm{U}\end{array}$ & \pm & $\begin{array}{l}{ }^{207} \mathrm{~Pb} / \\
{ }^{235} \mathrm{U}\end{array}$ & \pm & $\begin{array}{l}{ }^{207} \mathrm{~Pb} / \\
{ }^{206} \mathrm{~Pb}\end{array}$ & \pm & $\rho$ & $\begin{array}{l}{ }^{206} \mathrm{~Pb} / \\
{ }^{238} \mathrm{U}\end{array}$ & \pm & $\begin{array}{l}{ }^{207} \mathrm{~Pb} / \\
{ }^{206} \mathrm{~Pb}\end{array}$ & \pm & $\begin{array}{c}\% \\
\text { Disc }\end{array}$ \\
\hline 1.1 & 2004 & 88 & 0.04 & 282.2 & 0.000015 & 0.03 & 6.099 & 0.062 & 0.0715 & 0.0002 & 0.1641 & 0.0017 & 1.633 & 0.018 & 0.0722 & 0.0002 & 0.968 & 979 & 9 & 990 & 6 & 1 \\
\hline 2.1 & 91 & 58 & 0.64 & 10.7 & 0.000112 & 0.20 & 7.267 & 0.094 & 0.0673 & 0.0008 & 0.1373 & 0.0018 & 1.245 & 0.026 & 0.0657 & 0.0011 & 0.614 & 830 & 10 & 798 & 35 & -4 \\
\hline 3.1 & 103 & 104 & 1.01 & 18.1 & 0.000128 & 0.22 & 4.877 & 0.061 & 0.0795 & 0.0007 & 0.2046 & 0.0026 & 2.191 & 0.040 & 0.0777 & 0.0010 & 0.688 & 1200 & 14 & 1139 & 26 & -5 \\
\hline 4.1 & 339 & 164 & 0.48 & 55.2 & 0.000051 & 0.09 & 5.276 & 0.059 & 0.0751 & 0.0004 & 0.1904 & 0.0023 & 2.069 & 0.055 & 0.0788 & 0.0014 & 0.811 & 1123 & 12 & 1168 & 36 & 4 \\
\hline 5.1 & 438 & 90 & 0.21 & 177.4 & 0.000482 & 0.66 & 2.124 & 0.023 & 0.1849 & 0.0032 & 0.4677 & 0.0051 & 11.540 & 0.265 & 0.1790 & 0.0036 & 0.471 & 2473 & 22 & 2643 & 34 & 6 \\
\hline 6.1 & 435 & 438 & 1.01 & 52.2 & - & $<0.01$ & 7.156 & 0.078 & 0.0669 & 0.0004 & 0.1398 & 0.0015 & 1.293 & 0.016 & 0.0671 & 0.0004 & 0.867 & 843 & 9 & 840 & 13 & 0 \\
\hline 7.1 & 917 & 379 & 0.41 & 261.1 & 0.000006 & 0.01 & 3.018 & 0.031 & 0.1140 & 0.0003 & 0.3313 & 0.0034 & 5.203 & 0.055 & 0.1139 & 0.0003 & 0.975 & 1845 & 16 & 1862 & 4 & 1 \\
\hline 8.1 & 513 & 211 & 0.41 & 136.8 & 0.000023 & 0.04 & 3.223 & 0.034 & 0.1050 & 0.0003 & 0.3105 & 0.0034 & 4.515 & 0.078 & 0.1055 & 0.0009 & 0.925 & 1743 & 17 & 1723 & 15 & -1 \\
\hline 9.1 & 1288 & 982 & 0.76 & 146.6 & 0.000015 & 0.03 & 7.546 & 0.078 & 0.0658 & 0.0002 & 0.1325 & 0.0014 & 1.199 & 0.013 & 0.0656 & 0.0002 & 0.944 & 802 & 8 & 794 & 8 & -1 \\
\hline 10.1 & 140 & 176 & 1.25 & 37.4 & 0.000013 & 0.02 & 3.220 & 0.039 & 0.1054 & 0.0006 & 0.3105 & 0.0038 & 4.507 & 0.060 & 0.1053 & 0.0006 & 0.909 & 1743 & 18 & 1719 & 10 & -1 \\
\hline 11.1 & 1231 & 518 & 0.42 & 253.8 & 0.000013 & 0.02 & 4.167 & 0.043 & 0.0908 & 0.0002 & 0.2400 & 0.0025 & 2.998 & 0.032 & 0.0906 & 0.0002 & 0.975 & 1386 & 13 & 1438 & 4 & 4 \\
\hline 12.1 & 84 & 20 & 0.24 & 48.5 & - & $<0.01$ & 1.492 & 0.019 & 0.2599 & 0.0009 & 0.6704 & 0.0083 & 24.027 & 0.310 & 0.2599 & 0.0009 & 0.962 & 3307 & 32 & 3246 & 6 & -2 \\
\hline 13.1 & 667 & 130 & 0.19 & 100.8 & - & $<0.01$ & 5.686 & 0.206 & 0.0720 & 0.0011 & 0.1759 & 0.0064 & 1.745 & 0.068 & 0.0720 & 0.0011 & 0.925 & 1044 & 35 & 985 & 30 & -6 \\
\hline 14.1 & 189 & 74 & 0.39 & 20.6 & 0.000078 & 0.14 & 7.911 & 0.108 & 0.0677 & 0.0019 & 0.1262 & 0.0017 & 1.158 & 0.038 & 0.0665 & 0.0020 & 0.413 & 766 & 10 & 823 & 63 & 7 \\
\hline 15.1 & 1235 & 534 & 0.43 & 281.3 & 0.000172 & 0.30 & 3.772 & 0.040 & 0.0725 & 0.0004 & 0.2700 & 0.0030 & 3.257 & 0.076 & 0.0875 & 0.0015 & 0.750 & 1541 & 15 & 1372 & 32 & -12 \\
\hline 16.1 & 474 & 311 & 0.66 & 63.1 & 0.000314 & 0.54 & 6.445 & 0.070 & 0.0751 & 0.0005 & 0.1543 & 0.0017 & 1.501 & 0.027 & 0.0706 & 0.0010 & 0.609 & 925 & 9 & 944 & 29 & 2 \\
\hline 17.1 & 331 & 341 & 1.03 & 38.2 & 0.000020 & 0.03 & 7.443 & 0.080 & 0.0666 & 0.0004 & 0.1343 & 0.0014 & 1.228 & 0.015 & 0.0663 & 0.0004 & 0.858 & 812 & 8 & 816 & 13 & 0 \\
\hline 18.1 & 415 & 230 & 0.55 & 59.6 & 0.000010 & 0.02 & 5.981 & 0.064 & 0.0723 & 0.0003 & 0.1672 & 0.0018 & 1.663 & 0.019 & 0.0721 & 0.0004 & 0.909 & 996 & 10 & 990 & 10 & -1 \\
\hline 19.1 & 183 & 79 & 0.43 & 30.1 & 0.000016 & 0.03 & 5.214 & 0.059 & 0.0762 & 0.0005 & 0.1917 & 0.0022 & 2.008 & 0.027 & 0.0759 & 0.0005 & 0.854 & 1131 & 12 & 1094 & 14 & -3 \\
\hline 20.1 & 110 & 71 & 0.65 & 15.3 & 0.000049 & 0.08 & 6.181 & 0.076 & 0.0715 & 0.0007 & 0.1617 & 0.0020 & 1.577 & 0.026 & 0.0708 & 0.0008 & 0.752 & 966 & 11 & 951 & 22 & -2 \\
\hline 21.1 & 278 & 259 & 0.93 & 34.4 & 0.000106 & 0.18 & 6.935 & 0.076 & 0.0687 & 0.0005 & 0.1439 & 0.0016 & 1.334 & 0.019 & 0.0672 & 0.0006 & 0.755 & 867 & 9 & 844 & 20 & -3 \\
\hline 22.1 & 663 & 188 & 0.28 & 256.5 & 0.000009 & 0.01 & 2.222 & 0.023 & 0.1609 & 0.0003 & 0.4500 & 0.0046 & 9.978 & 0.104 & 0.1608 & 0.0003 & 0.987 & 2395 & 21 & 2464 & 3 & 3 \\
\hline 23.1 & 168 & 96 & 0.57 & 38.3 & 0.000108 & 0.17 & 3.764 & 0.043 & 0.0961 & 0.0006 & 0.2652 & 0.0030 & 3.460 & 0.061 & 0.0946 & 0.0013 & 0.642 & 1516 & 15 & 1520 & 26 & 0 \\
\hline 24.1 & 439 & 415 & 0.94 & 50.3 & 0.000221 & 0.39 & 7.508 & 0.079 & 0.0681 & 0.0003 & 0.1327 & 0.0014 & 1.188 & 0.020 & 0.0649 & 0.0009 & 0.625 & 803 & 8 & 773 & 28 & -4 \\
\hline 25.1 & 19 & 30 & 1.55 & 7.2 & - & $<0.01$ & 2.256 & 0.042 & 0.1598 & 0.0020 & 0.4433 & 0.0082 & 9.764 & 0.217 & 0.1598 & 0.0020 & 0.832 & 2365 & 37 & 2453 & 21 & 4 \\
\hline 26.1 & 354 & 198 & 0.56 & 88.1 & 0.000014 & 0.02 & 3.457 & 0.038 & 0.0988 & 0.0004 & 0.2892 & 0.0031 & 3.932 & 0.045 & 0.0986 & 0.0004 & 0.946 & 1637 & 16 & 1598 & 7 & -2 \\
\hline 27.1 & 999 & 230 & 0.23 & 139.0 & 0.000003 & 0.01 & 6.175 & 0.065 & 0.0711 & 0.0003 & 0.1619 & 0.0017 & 1.586 & 0.018 & 0.0710 & 0.0003 & 0.944 & 968 & 9 & 958 & 7 & -1 \\
\hline 28.1 & 159 & 98 & 0.62 & 27.3 & 0.000075 & 0.13 & 5.005 & 0.081 & 0.0820 & 0.0006 & 0.1996 & 0.0032 & 2.227 & 0.041 & 0.0809 & 0.0007 & 0.875 & 1173 & 17 & 1220 & 18 & 4 \\
\hline 29.1 & not compl & leted & & & & & & & & & & & & & & & & & & & & \\
\hline 30.1 & 32 & 59 & 1.84 & 9.4 & 0.000130 & 0.21 & 2.963 & 0.053 & 0.1035 & 0.0025 & 0.3368 & 0.0060 & 4.720 & 0.150 & 0.1017 & 0.0027 & 0.563 & 1871 & 29 & 1655 & 49 & -13 \\
\hline 31.1 & 362 & 69 & 0.19 & 64.9 & 0.000017 & 0.03 & 4.797 & 0.281 & 0.0906 & 0.0024 & 0.2084 & 0.0122 & 2.596 & 0.167 & 0.0904 & 0.0024 & 0.910 & 1220 & 65 & 1433 & 51 & 15 \\
\hline 32.1 & 358 & 245 & 0.68 & 50.5 & 0.000018 & 0.03 & 6.087 & 0.067 & 0.0717 & 0.0004 & 0.1642 & 0.0018 & 1.617 & 0.020 & 0.0714 & 0.0004 & 0.887 & 980 & 10 & 969 & 12 & -1 \\
\hline 33.1 & 116 & 81 & 0.70 & 32.1 & 0.000183 & 0.29 & 3.103 & 0.037 & 0.1091 & 0.0042 & 0.3214 & 0.0039 & 4.722 & 0.201 & 0.1066 & 0.0044 & 0.282 & 1797 & 19 & 1742 & 75 & -3 \\
\hline 34.1 & 195 & 187 & 0.96 & 24.6 & 0.000090 & 0.16 & 6.830 & 0.079 & 0.0669 & 0.0018 & 0.1462 & 0.0017 & 1.322 & 0.040 & 0.0656 & 0.0018 & 0.380 & 880 & 9 & 793 & 59 & -11 \\
\hline 35.1 & 716 & 476 & 0.66 & 282.5 & - & $<0.01$ & 2.178 & 0.023 & 0.1619 & 0.0010 & 0.4591 & 0.0048 & 10.251 & 0.126 & 0.1619 & .0010 & .855 & 2436 & 21 & 2476 & 11 & 2 \\
\hline 36.1 & 196 & 135 & 0.69 & 38.0 & - & $<0.01$ & 4.437 & 0.052 & 0.0784 & 0.0025 & 0.2255 & 0.0027 & 2.447 & 0.083 & 0.0787 & 0.0025 & 0.3 & 1311 & 14 & 1165 & 63 & -13 \\
\hline 37.1 & 506 & 200 & 0.39 & 78.7 & 0.000028 & 0.05 & 5.520 & 0.115 & 0.0704 & 0.0003 & 0.1811 & 0.0038 & 1.748 & 0.037 & 0.0700 & 0.0004 & 0.971 & 1073 & 21 & 928 & 11 & -16 \\
\hline 38.1 & 154 & 92 & 0.59 & 44.6 & 0.000009 & 0.01 & 2.967 & 0.074 & 0.1203 & 0.0032 & 0.3370 & 0.0085 & 5.587 & 0.205 & 0.1202 & 0.0032 & 0.685 & 1872 & 41 & 1960 & 48 & 4 \\
\hline 39.1 & 218 & 153 & 0.70 & 25.6 & - & $<0.01$ & 7.317 & 0.087 & 0.0657 & 0.0007 & 0.1367 & 0.0016 & 1.241 & 0.019 & 0.0658 & .0007 & 0.762 & 826 & 9 & 801 & 21 & -3 \\
\hline 40.1 & 417 & 152 & 0.36 & 78.0 & $\cdot$ & $<0.01$ & 4.592 & 0.049 & 0.0858 & 0.0014 & 0.2178 & 0.0023 & 2.584 & 0.051 & 0.0860 & .0014 & 0.540 & 1270 & 12 & 1339 & 32 & 5 \\
\hline 41.1 & 353 & 177 & 0.50 & 79.3 & 0.000028 & 0.05 & 3.822 & 0.042 & 0.0924 & 0.0004 & 0.2615 & 0.0029 & 3.316 & 0.039 & 0.0920 & 0.0004 & 0.929 & 1498 & 15 & 1467 & 8 & -2 \\
\hline 42.1 & 529 & 113 & 0.21 & 77.3 & 0.000049 & 0.08 & 5.880 & 0.063 & 0.0718 & 0.0003 & 0.1705 & 0.0019 & 1.739 & 0.032 & 0.0740 & 0.0009 & 0.814 & 1015 & 10 & 1041 & 23 & 2 \\
\hline 43.1 & 175 & 66 & 0.38 & 40.6 & 0.000036 & 0.06 & 3.701 & 0.123 & 0.0975 & 0.0057 & 0.2701 & 0.0089 & 3.612 & 0.243 & 0.0970 & .0057 & 0.492 & 1541 & 45 & 1567 & 110 & 2 \\
\hline 44.1 & 289 & 121 & 0.42 & 87.0 & - & $<0.01$ & 2.853 & 0.063 & 0.1120 & 0.0040 & 0.3506 & 0.0078 & 5.419 & 0.227 & 0.1121 & .0040 & 0.529 & 1937 & 37 & 1834 & 64 & -6 \\
\hline 45.1 & 149 & 24 & 0.16 & 29.5 & 0.000056 & 0.10 & 4.341 & 0.058 & 0.0737 & 0.0007 & 0.2306 & 0.0032 & 2.372 & 0.071 & 0.0746 & 0.0017 & 0.662 & 1338 & 17 & 1058 & 46 & -26 \\
\hline 46.1 & 791 & 189 & 0.24 & 162.3 & 0.000007 & 0.01 & 4.187 & 0.069 & 0.0669 & 0.0024 & 0.2401 & 0.0042 & 2.364 & 0.130 & 0.0714 & 0.0035 & 0.520 & 1387 & 22 & 969 & 99 & -43 \\
\hline 47.1 & 123 & 204 & 1.66 & 12.3 & - & $<0.01$ & 8.629 & 0.197 & 0.0635 & 0.0011 & 0.1159 & 0.0026 & 1.015 & 0.029 & 0.0635 & 0.0011 & 0.795 & 707 & 15 & 726 & 37 & 3 \\
\hline 48.1 & 972 & 597 & 0.61 & 175.1 & 0.000011 & 0.02 & 4.769 & 0.069 & 0.0804 & 0.0006 & 0.2097 & 0.0030 & 2.319 & 0.038 & 0.0802 & .0006 & 0.884 & 1227 & 16 & 1202 & 15 & -2 \\
\hline 49.1 & 1748 & 190 & 0.11 & 227.7 & 0.000034 & 0.06 & 6.596 & 0.068 & 0.0840 & 0.0004 & 0.1515 & 0.0016 & 1.744 & 0.020 & 0.0835 & 0.0004 & 0.914 & 909 & 9 & 1281 & 9 & 29 \\
\hline 50.1 & 320 & 234 & 0.73 & 54.0 & - & $<0.01$ & 5.093 & 0.055 & 0.0782 & 0.0004 & 0.1964 & 0.0021 & 2.118 & 0.025 & 0.0782 & 0.0004 & 0.910 & 1156 & 11 & 1153 & 10 & 0 \\
\hline 51.1 & 225 & 201 & 0.89 & 31.2 & 0.000015 & 0.03 & 6.202 & 0.069 & 0.0727 & 0.0005 & 0.1612 & 0.0018 & 1.611 & 0.021 & 0.0725 & .0005 & 0.845 & 963 & 10 & 1000 & 14 & 4 \\
\hline 52.1 & 253 & 203 & 0.80 & 29.3 & 0.000144 & 0.25 & 7.398 & 0.083 & 0.0667 & 0.0006 & 0.1348 & 0.0015 & 1.200 & 0.020 & 0.0646 & .0008 & 0.660 & 815 & 9 & 761 & 27 & -7 \\
\hline 53.1 & 290 & 142 & 0.49 & 80.4 & - & $<0.01$ & 3.097 & 0.034 & 0.1083 & 0.0004 & 0.3229 & 0.0035 & 4.822 & 0.056 & 0.1083 & .0004 & 0.94 & 1804 & 17 & 1771 & 7 & -2 \\
\hline 54.1 & 616 & 299 & 0.49 & 80.1 & 0.000016 & 0.03 & 6.600 & 0.071 & 0.0695 & 0.0007 & 0.1515 & 0.0016 & 1.446 & 0.022 & 0.0692 & 0.0007 & 0.719 & 909 & 9 & 905 & 21 & 0 \\
\hline 55.1 & 138 & 134 & 0.97 & 15.9 & 0.000009 & 0.02 & 7.453 & 0.090 & 0.0670 & 0.0007 & 0.1342 & 0.0016 & 1.237 & 0.020 & 0.0669 & 0.0008 & 0.734 & 812 & 9 & 833 & 23 & 3 \\
\hline 56.1 & 1046 & 287 & 0.27 & 140.6 & 0.000022 & 0.04 & 6.394 & 0.066 & 0.0716 & 0.0002 & 0.1563 & 0.0016 & 1.536 & 0.017 & 0.0713 & .0003 & 0.941 & 936 & 9 & 965 & 8 & 3 \\
\hline 57.1 & 1110 & 668 & 0.60 & 242.1 & 0.000002 & $<0.01$ & 3.940 & 0.040 & 0.0912 & 0.0002 & 0.2538 & 0.0026 & 3.189 & 0.033 & 0.0912 & .0002 & 0.975 & 1458 & 13 & 1450 & 4 & -1 \\
\hline 58.1 & 1198 & 85 & 0.07 & 166.9 & 0.000016 & 0.03 & 6.166 & 0.097 & 0.0713 & 0.0005 & 0.1621 & 0.0026 & 1.589 & 0.028 & 0.0711 & . 0005 & 0.905 & 969 & 14 & 960 & 15 & -1 \\
\hline 59.1 & 556 & 177 & 0.32 & 146.2 & 0.000007 & 0.01 & 3.265 & 0.035 & 0.1056 & 0.0003 & 0.3063 & 0.0033 & 4.456 & 0.049 & 0.1055 & 0.0003 & 0.966 & 1722 & 16 & 1723 & 5 & 0 \\
\hline 60.1 & 124 & 69 & 0.56 & 23.1 & 0.000362 & 0.61 & 4.603 & 0.056 & 0.0836 & 0.0007 & 0.2164 & 0.0029 & 2.397 & 0.077 & 0.0803 & .0018 & 0.817 & 1263 & 15 & 1205 & 45 & -5 \\
\hline 61.1 & 204 & 130 & 0.63 & 29.8 & 0.000038 & 0.07 & 5.894 & 0.067 & 0.0732 & 0.0005 & 0.1695 & 0.0019 & 1.699 & 0.024 & 0.0727 & .0006 & 0.812 & 1010 & 11 & 1005 & 17 & -1 \\
\hline 62.1 & 406 & 57 & 0.14 & 74.1 & 0.000013 & 0.02 & 4.705 & 0.051 & 0.0819 & 0.0004 & 0.2125 & 0.0023 & 2.393 & 0.028 & 0.0817 & .0004 & 0.926 & 1242 & 12 & 1238 & 9 & 0 \\
\hline 63.1 & 153 & 151 & 0.99 & 37.2 & 0.000001 & $<0.01$ & 3.530 & 0.041 & 0.1005 & 0.0008 & 0.2832 & 0.0033 & 3.923 & 0.055 & 0.1005 & 0.0008 & 0.831 & 1608 & 16 & 1633 & 14 & 2 \\
\hline 64.1 & 578 & 113 & 0.19 & 98.9 & 0.000017 & 0.03 & 5.021 & 0.054 & 0.0778 & 0.0004 & 0.1991 & 0.0021 & 2.129 & 0.025 & 0.0776 & 0.0004 & 0.908 & 1171 & 11 & 1135 & 10 & -3 \\
\hline 65.1 & 441 & 252 & 0.57 & 51.9 & 0.000014 & 0.02 & 7.296 & 0.080 & 0.0677 & 0.0005 & 0.1370 & 0.0015 & 1.275 & 0.017 & 0.0675 & 0.0005 & 0.841 & 828 & 9 & 852 & 15 & 3 \\
\hline 66.1 & 318 & 114 & 0.36 & 47.0 & 0.000018 & 0.03 & 5.810 & 0.065 & 0.0752 & 0.0005 & 0.1721 & 0.0019 & 1.777 & 0.024 & 0.0749 & 0.0005 & 0.846 & 1024 & 11 & 1066 & 14 & 4 \\
\hline
\end{tabular}

Notes : $\quad$ 1. Uncertainties given at the one $\sigma$ level.

2. Error in Temora reference zircon calibration was $0.38 \%$ for the analytical session.

( not included in above errors but required when comparing ${ }^{206} \mathrm{~Pb} /{ }^{238} \mathrm{U}$ data from different mounts)

3. $\mathrm{f}_{206} \%$ denotes the percentage of ${ }^{206} \mathrm{~Pb}$ that is common $\mathrm{Pb}$.

4. Correction for common $\mathrm{Pb}$ made using the measured ${ }^{204} \mathrm{~Pb} / 200 \mathrm{~Pb}$ ratio.

5. For $\%$ Disc, $0 \%$ denotes a concordant analysis. 
Table 3. SHRIMP U-Pb data for zircon grains from sample Tal

\begin{tabular}{|c|c|c|c|c|c|c|c|c|c|c|c|c|c|c|c|c|c|c|c|c|c|c|}
\hline \multirow[b]{2}{*}{$\begin{array}{c}\text { Grain. } \\
\text { spot }\end{array}$} & \multirow[b]{2}{*}{$\underset{(\mathrm{ppm})}{\mathrm{U}}$} & \multirow[b]{2}{*}{$\begin{array}{c}\text { Th } \\
(\mathrm{ppm})\end{array}$} & \multirow[b]{2}{*}{$\mathrm{Th} / \mathrm{U}$} & \multirow[b]{2}{*}{$\begin{array}{l}{ }^{206} \mathrm{~Pb}^{*} \\
(\mathrm{ppm})\end{array}$} & & & & Total $\mathrm{R}$ & atios & & & & adiogeni & Ratios & & & & & & e (Ma) & & \\
\hline & & & & & $\begin{array}{l}{ }^{204} \mathrm{~Pb} / \\
{ }^{206} \mathrm{~Pb}\end{array}$ & $\begin{array}{l}\mathrm{f}_{206} \\
\%\end{array}$ & $\begin{array}{l}{ }^{238} \mathrm{U} / \\
{ }^{206} \mathrm{~Pb}\end{array}$ & \pm & $\begin{array}{l}{ }^{207} \mathrm{~Pb} / \\
{ }^{206} \mathrm{~Pb}\end{array}$ & \pm & $\begin{array}{l}{ }^{206} \mathrm{~Pb} / \\
{ }^{238} \mathrm{U}\end{array}$ & \pm & $\begin{array}{c}{ }^{207} \mathrm{~Pb} / \\
{ }^{235} \mathrm{U}\end{array}$ & \pm & $\begin{array}{l}{ }^{207} \mathrm{~Pb} / \\
{ }^{206} \mathrm{~Pb}\end{array}$ & \pm & $\rho$ & $\begin{array}{l}{ }^{206} \mathrm{~Pb} / \\
{ }^{238} \mathrm{U}\end{array}$ & \pm & $\begin{array}{l}{ }^{207} \mathrm{~Pb} / \\
{ }^{206} \mathrm{~Pb}\end{array}$ & \pm & $\begin{array}{c}\% \\
\text { Disc }\end{array}$ \\
\hline 1.1 & 184 & 70 & 0.38 & 33 & 0.000174 & 0.26 & 4.749 & 0.062 & 0.1267 & 0.0112 & 0.2100 & 0.0028 & 3.602 & 0.329 & 0.1244 & 0.0112 & 0.145 & 1229 & 15 & 2020 & 160 & 39 \\
\hline 2.1 & 593 & 352 & 0.59 & 143 & 0.000013 & 0.02 & 3.569 & 0.040 & 0.0982 & 0.0006 & 0.2802 & 0.0031 & 3.785 & 0.048 & 0.0980 & 0.0006 & 0.879 & 1592 & 16 & 1586 & 11 & 0 \\
\hline 3.1 & 63 & 95 & 1.51 & 18 & 0.000026 & 0.04 & 3.077 & 0.056 & 0.1075 & 0.0017 & 0.3249 & 0.0059 & 4.800 & 0.118 & 0.1072 & 0.0018 & 0.738 & 1814 & 29 & 1752 & 30 & -4 \\
\hline 4.1 & 579 & 311 & 0.54 & 82 & 0.000014 & 0.02 & 6.026 & 0.068 & 0.0726 & 0.0006 & 0.1659 & 0.0019 & 1.655 & 0.024 & 0.0724 & 0.0007 & 0.780 & 989 & 10 & 997 & 18 & 1 \\
\hline 5.1 & 163 & 141 & 0.86 & 32 & 0.000034 & 0.06 & 4.404 & 0.062 & 0.0858 & 0.0011 & 0.2269 & 0.0032 & 2.668 & 0.054 & 0.0853 & 0.0012 & 0.699 & 1318 & 17 & 1322 & 28 & 0 \\
\hline 6.1 & 220 & 127 & 0.58 & 117 & 0.000027 & 0.04 & 1.618 & 0.021 & 0.1472 & 0.0013 & 0.6178 & 0.0080 & 12.509 & 0.199 & 0.1468 & 0.0013 & 0.816 & 3101 & 32 & 2310 & 16 & -34 \\
\hline 7.1 & 52 & 49 & 0.94 & 11 & 0.000040 & 0.06 & 3.980 & 0.074 & 0.0942 & 0.0019 & 0.2511 & 0.0047 & 3.242 & 0.092 & 0.0936 & 0.0020 & 0.660 & 1444 & 24 & 1500 & 40 & 4 \\
\hline 8.1 & 531 & 217 & 0.41 & 150 & - & $<0.01$ & 3.050 & 0.035 & 0.1126 & 0.0006 & 0.3279 & 0.0037 & 5.095 & 0.064 & 0.1127 & 0.0006 & 0.906 & 1828 & 18 & 1843 & 10 & 1 \\
\hline 9.1 & 223 & 206 & 0.92 & 60 & 0.000088 & 0.14 & 3.195 & 0.040 & 0.1092 & 0.0009 & 0.3125 & 0.0039 & 4.653 & 0.074 & 0.1080 & 0.0010 & 0.793 & 1753 & 19 & 1766 & 18 & 1 \\
\hline 10.1 & 215 & 164 & 0.77 & 54 & 0.000040 & 0.06 & 3.402 & 0.043 & 0.1048 & 0.0011 & 0.2938 & 0.0037 & 4.223 & 0.071 & 0.1043 & 0.0011 & 0.761 & 1660 & 19 & 1701 & 20 & 2 \\
\hline 11.1 & 279 & 153 & 0.55 & 66 & - & $<0.01$ & 3.641 & 0.045 & 0.0960 & 0.0008 & 0.2747 & 0.0034 & 3.650 & 0.055 & 0.0964 & 0.0008 & 0.817 & 1565 & 17 & 1555 & 16 & -1 \\
\hline 12.1 & 37 & 26 & 0.70 & 10 & 0.000700 & 1.12 & 3.124 & 0.065 & 0.1062 & 0.0022 & 0.3165 & 0.0069 & 4.211 & 0.255 & 0.0965 & 0.0055 & 0.358 & 1773 & 34 & 1557 & 106 & -14 \\
\hline 13.1 & 258 & 139 & 0.54 & 73 & 0.000045 & 0.07 & 3.059 & 0.038 & 0.1086 & 0.0009 & 0.3267 & 0.0041 & 4.866 & 0.077 & 0.1080 & 0.0011 & 0.788 & 1822 & 20 & 1766 & 18 & -3 \\
\hline 14.1 & 432 & 413 & 0.96 & 48 & 0.000023 & 0.04 & 7.702 & 0.090 & 0.0656 & 0.0008 & 0.1298 & 0.0015 & 1.167 & 0.020 & 0.0652 & 0.0008 & 0.682 & 787 & 9 & 782 & 26 & -1 \\
\hline 15.1 & 215 & 202 & 0.94 & 59 & 0.000021 & 0.03 & 3.151 & 0.040 & 0.1043 & 0.0011 & 0.3173 & 0.0040 & 4.550 & 0.076 & 0.1040 & 0.0011 & 0.755 & 1776 & 20 & 1697 & 20 & -5 \\
\hline 16.1 & 85 & 52 & 0.62 & 23 & 0.000106 & 0.17 & 3.191 & 0.051 & 0.1086 & 0.0015 & 0.3129 & 0.0050 & 4.625 & 0.104 & 0.1072 & 0.0017 & 0.718 & 1755 & 25 & 1752 & 29 & 0 \\
\hline 17.1 & 789 & 276 & 0.35 & 133 & 0.000019 & 0.03 & 5.104 & 0.056 & 0.0835 & 0.0005 & 0.1959 & 0.0021 & 2.248 & 0.029 & 0.0832 & 0.0005 & 0.860 & 1153 & 12 & 1275 & 13 & 10 \\
\hline 18.1 & 1687 & 130 & 0.08 & 251 & 0.000022 & 0.04 & 5.771 & 0.060 & 0.0911 & 0.0004 & 0.1732 & 0.0018 & 2.167 & 0.025 & 0.0908 & 0.0004 & 0.908 & 1030 & 10 & 1441 & 9 & 29 \\
\hline 19.1 & 165 & 130 & 0.78 & 46 & 0.000005 & 0.01 & 3.066 & 0.041 & 0.1079 & 0.0010 & 0.3261 & 0.0044 & 4.849 & 0.079 & 0.1078 & 0.0010 & 0.818 & 1820 & 21 & 1763 & 17 & -3 \\
\hline 20.1 & 269 & 169 & 0.63 & 71 & 0.000065 & 0.10 & 3.239 & 0.039 & 0.1053 & 0.0008 & 0.3084 & 0.0037 & 4.441 & 0.064 & 0.1044 & 0.0008 & 0.827 & 1733 & 18 & 1704 & 15 & -2 \\
\hline 21.1 & 107 & 168 & 1.57 & 23 & 0.000115 & 0.19 & 3.989 & 0.061 & 0.0904 & 0.0013 & 0.2502 & 0.0038 & 3.062 & 0.085 & 0.0888 & 0.0020 & 0.554 & 1439 & 20 & 1399 & 44 & -3 \\
\hline 22.1 & 218 & 223 & 1.02 & 50 & 0.000007 & 0.01 & 3.721 & 0.047 & 0.0949 & 0.0009 & 0.2687 & 0.0034 & 3.513 & 0.056 & 0.0948 & 0.0009 & 0.798 & 1534 & 17 & 1524 & 18 & -1 \\
\hline 23.1 & 302 & 211 & 0.70 & 35 & 0.000096 & 0.17 & 7.465 & 0.094 & 0.0670 & 0.0009 & 0.1337 & 0.0017 & 1.210 & 0.030 & 0.0656 & 0.0014 & 0.504 & 809 & 10 & 794 & 46 & -2 \\
\hline 24.1 & 243 & 130 & 0.53 & 40 & 0.000088 & 0.15 & 5.174 & 0.066 & 0.0767 & 0.0009 & 0.1930 & 0.0025 & 2.008 & 0.048 & 0.0755 & 0.0015 & 0.535 & 1137 & 13 & 1081 & 41 & -5 \\
\hline 25.1 & 165 & 65 & 0.39 & 35 & - & $<0.01$ & 4.044 & 0.055 & 0.0892 & 0.0010 & 0.2474 & 0.0034 & 3.057 & 0.057 & 0.0896 & 0.0011 & 0.735 & 1425 & 17 & 1418 & 24 & -1 \\
\hline 26.1 & 175 & 164 & 0.94 & 20 & 0.000001 & $<0.01$ & 7.534 & 0.105 & 0.0669 & 0.0012 & 0.1327 & 0.0018 & 1.224 & 0.027 & 0.0669 & 0.0012 & 0.623 & 803 & 10 & 834 & 36 & 4 \\
\hline 27.1 & 33 & 36 & 1.09 & 4 & - & $<0.01$ & 7.710 & 0.186 & 0.0724 & 0.0028 & 0.1307 & 0.0032 & 1.422 & 0.109 & 0.0789 & 0.0057 & 0.325 & 792 & 19 & 1169 & 143 & 32 \\
\hline 28.1 & 313 & 163 & 0.52 & 33 & 0.000006 & 0.01 & 8.135 & 0.101 & 0.0652 & 0.0009 & 0.1229 & 0.0015 & 1.104 & 0.020 & 0.0651 & 0.0009 & 0.672 & 747 & 9 & 779 & 29 & 4 \\
\hline 29.1 & 23 & 13 & 0.58 & 5 & - & $<0.01$ & 3.670 & 0.095 & 0.1311 & 0.0032 & 0.2725 & 0.0071 & 4.927 & 0.176 & 0.1311 & 0.0032 & 0.728 & 1553 & 36 & 2113 & 43 & 26 \\
\hline 30.1 & 36 & 34 & 0.96 & 15 & 0.000193 & 0.27 & 2.092 & 0.044 & 0.1618 & 0.0022 & 0.4767 & 0.0101 & 10.476 & 0.279 & 0.1594 & 0.0026 & 0.793 & 2513 & 44 & 2449 & 27 & -3 \\
\hline 31.1 & 64 & 76 & 1.19 & 7 & 0.000292 & 0.51 & 7.811 & 0.147 & 0.0680 & 0.0020 & 0.1274 & 0.0024 & 1.121 & 0.065 & 0.0638 & 0.0035 & 0.328 & 773 & 14 & 736 & 117 & -5 \\
\hline 32.1 & 471 & 381 & 0.81 & 125 & 0.000029 & 0.05 & 3.225 & 0.036 & 0.1105 & 0.0006 & 0.3099 & 0.0035 & 4.704 & 0.060 & 0.1101 & 0.0006 & 0.886 & 1740 & 17 & 1801 & 11 & 3 \\
\hline 33.1 & 512 & 173 & 0.34 & 68 & - & $<0.01$ & 6.507 & 0.075 & 0.0693 & 0.0006 & 0.1538 & 0.0018 & 1.478 & 0.022 & 0.0697 & 0.0007 & 0.768 & 922 & 10 & 920 & 20 & 0 \\
\hline 34.1 & 154 & 97 & 0.63 & 21 & 0.000026 & 0.04 & 6.423 & 0.090 & 0.0708 & 0.0012 & 0.1556 & 0.0022 & 1.512 & 0.033 & 0.0705 & 0.0012 & 0.637 & 932 & 12 & 942 & 35 & 1 \\
\hline 35.1 & 396 & 130 & 0.33 & 70 & 0.000052 & 0.09 & 4.889 & 0.057 & 0.0883 & 0.0020 & 0.2043 & 0.0024 & 2.467 & 0.064 & 0.0876 & 0.0020 & 0.447 & 1199 & 13 & 1373 & 45 & 13 \\
\hline 36.1 & 208 & 88 & 0.42 & 58 & 0.000020 & 0.03 & 3.095 & 0.039 & 0.1086 & 0.0009 & 0.3230 & 0.0040 & 4.825 & 0.073 & 0.1083 & 0.0009 & 0.827 & 1804 & 20 & 1772 & 15 & -2 \\
\hline 37.1 & 145 & 74 & 0.51 & 8 & 0.000449 & 0.72 & 15.382 & 0.248 & 0.0606 & 0.0017 & 0.0645 & 0.0011 & & & & & & 403 & 6 & & & \\
\hline 38.1 & 411 & 127 & 0.31 & 134 & - & $<0.01$ & 2.632 & 0.030 & 0.1283 & 0.0007 & 0.3799 & 0.0044 & 6.721 & 0.084 & 0.1283 & 0.0007 & 0.913 & 2076 & 20 & 2075 & 9 & 0 \\
\hline 39.1 & 135 & 85 & 0.63 & 17 & - & $<0.01$ & 6.842 & 0.101 & 0.0712 & 0.0013 & 0.1462 & 0.0022 & 1.443 & 0.035 & 0.0716 & 0.0013 & 0.616 & 880 & 12 & 973 & 38 & 10 \\
\hline 40.1 & 188 & 153 & 0.81 & 27 & 0.000018 & 0.03 & 6.106 & 0.082 & 0.0717 & 0.0010 & 0.1637 & 0.0022 & 1.612 & 0.032 & .0714 & 0.0011 & 0.669 & 977 & 12 & 969 & 30 & -1 \\
\hline 41.1 & 204 & 75 & 0.37 & 27 & 000155 & 0.27 & 6.516 & 0.090 & 0.0725 & 0.0013 & 0.1531 & 0.0021 & 1.483 & 0.039 & 0703 & 0.0016 & 0.521 & 918 & 12 & 937 & 47 & 2 \\
\hline 42.1 & 237 & 143 & 0.61 & 29 & 000156 & 0.27 & 6.942 & 0.091 & 0.0706 & 0.0010 & 0.1437 & 0.0019 & 1.354 & 0.039 & .0684 & 0.0018 & 0.453 & & 11 & 87 & 54 & 2 \\
\hline 43.1 & 77 & 59 & 0.77 & 7 & 0.000255 & 0.44 & 8.925 & 0.160 & 0.0711 & 0.0020 & 0.1116 & 0.0021 & 1.037 & 0.063 & 0.0674 & 0.0039 & 0.303 & 682 & 12 & 851 & 120 & 20 \\
\hline 44.1 & 137 & 100 & 0.73 & 33 & 0.000122 & 0.19 & 3.624 & 0.051 & 0.1191 & 0.0012 & 0.2755 & 0.0039 & 4.462 & 0.083 & 11175 & 0.0014 & 0.753 & 1568 & 19 & 1918 & 22 & 18 \\
\hline 45.1 & 376 & 141 & 0.38 & 104 & 000081 & 0.13 & 3.098 & .036 & 0.1108 & 0.0007 & 0.3224 & .0038 & 4.875 & 0.067 & 1097 & 0.0008 & 0.841 & 1801 & 18 & 1794 & 14 & 0 \\
\hline 46.1 & 274 & 75 & 0.27 & 73 & 000012 & 0.02 & 3.215 & 0.039 & 0.1064 & 0.0008 & 0.3109 & 0.0037 & 4.554 & 0.065 & .1062 & 0.0008 & 0.846 & 1745 & 18 & 1736 & 14 & -1 \\
\hline 47.1 & 304 & 324 & 1.07 & 51 & 0.000010 & 0.02 & 5.073 & 0.062 & 0.0797 & 0.0009 & 0.1971 & 0.0024 & 2.162 & 0.036 & 0.0795 & 0.0009 & 0.735 & 1160 & 13 & 1186 & 22 & 2 \\
\hline 48.1 & 379 & 471 & 1.24 & 95 & 000038 & 0.06 & 3.418 & 0.039 & 0.1047 & 0.0007 & 0.2924 & 0.0034 & 4.199 & 0.057 & 1042 & 0.0007 & 0.851 & 1654 & 17 & 1699 & 13 & 3 \\
\hline 49.1 & 301 & 306 & 1.02 & 32 & 000079 & 0.14 & 8.029 & 101 & 0.0667 & 0.0009 & 0.1244 & 0.0016 & 1.125 & 0.025 & 0656 & 0.0012 & 0.561 & 756 & 9 & 793 & 39 & 5 \\
\hline 50.1 & 167 & 27 & 0.16 & 22 & 000078 & 0.14 & 6.618 & 0.092 & 0.0695 & 0.0011 & 0.1509 & 0.0021 & 1.422 & 0.036 & .0683 & 0.0014 & 0.550 & 906 & 12 & 879 & 44 & -3 \\
\hline 51.1 & 252 & 77 & 0.31 & 71 & 000079 & 0.12 & 3.074 & 038 & 0.1116 & 0.0008 & 0.3249 & .0040 & 4.951 & 0.074 & .1105 & 0.0010 & 0.818 & 1814 & 19 & 1808 & 16 & 0 \\
\hline 52.1 & 82 & 64 & 0.77 & 24 & 0.000057 & 0.09 & 2.920 & 0.047 & 0.0992 & 0.0016 & 0.3422 & 0.0055 & 4.644 & 0.114 & 0.0984 & 0.0018 & 0.656 & 1897 & 26 & 1595 & 35 & -19 \\
\hline 53.1 & 584 & 47 & 0.08 & 82 & & 0.05 & & .071 & 0.0793 & 0030 & 1635 & 0019 & 1.779 & 0.071 & 0789 & .0030 & 0.290 & 976 & 10 & 1170 & 75 & 17 \\
\hline 54.1 & 132 & 105 & 0.79 & 39 & 0.000022 & & 2.8 & .040 & 0.1182 & 0.0011 & 0.3451 & .0048 & 5.610 & 0.096 & 1179 & 0.0012 & 0.812 & 1911 & 23 & 1925 & 18 & 1 \\
\hline 55.1 & 243 & 169 & 0.69 & 58 & - & $<0.01$ & 3.580 & .044 & 0.0984 & 0.0008 & 0.2795 & 0.0035 & 3.803 & 0.058 & .0987 & 0.0009 & 0.810 & 1589 & 18 & 1600 & 17 & 1 \\
\hline 56.1 & 282 & 197 & 0.70 & 51 & - & $<0.01$ & 4.742 & 0.057 & 0.0965 & 0.0009 & 0.2109 & 0.0025 & 2.809 & 0.043 & .0966 & 0.0009 & 0.790 & 1234 & 14 & 1559 & 18 & 21 \\
\hline 57.1 & 366 & 85 & 0.23 & 53 & 0.0000 & 0.05 & & .070 & 0.0722 & .0009 & 0.1698 & .0020 & 1.681 & 0.031 & 7718 & 0.0010 & 0.650 & 1011 & 11 & 980 & 28 & -3 \\
\hline 58.1 & 290 & 97 & 0.34 & 82 & & & & 036 & .1408 & & 3304 & & 6.390 & 0.095 & 1403 & 0.0012 & 0.812 & 1840 & 19 & 2231 & 15 & 17 \\
\hline 59.1 & 204 & 93 & 0.46 & 52 & 0.000028 & 0.04 & & .043 & 0.1036 & 0.0009 & 0.2991 & 0.0039 & 4.255 & 0.069 & 1032 & 0.0010 & 0.794 & 1687 & 19 & 1682 & 18 & 0 \\
\hline 60.1 & 558 & 456 & 0.82 & 108 & 0.000044 & 0.07 & 4.427 & 0.050 & 0.0875 & 0.0006 & 0.2257 & 0.0025 & 2.702 & 0.038 & 0.0868 & 0.0007 & 0.808 & 1312 & 13 & 1357 & 16 & 3 \\
\hline 61.1 & 258 & 178 & 0.69 & 29 & - & $<0.01$ & 7.611 & 0.097 & 0.0666 & 0.0010 & 0.1315 & 0.0017 & 1.227 & 0.030 & 0.0677 & 0.0014 & 0.525 & 797 & 10 & 858 & 43 & 7 \\
\hline 62.1 & 152 & 219 & 1.44 & 38 & 0 & 0.01 & & & & & 2883 & & 3.979 & 0.069 & 0.1001 & 0.0011 & 0.783 & 1633 & 19 & 1626 & 20 & 0 \\
\hline 63.1 & 135 & 58 & 0.43 & 40 & 0.000031 & 0.05 & 2.883 & 0.040 & 0.1154 & 0.0011 & 0.3467 & 0.0048 & 5.496 & 0.094 & 0.1150 & 0.0012 & 0.809 & 1919 & 23 & 1880 & 18 & -2 \\
\hline 64.1 & 647 & 115 & 0.18 & 92 & - & $<0.01$ & 6.066 & 0.067 & 0.0709 & 0.0006 & 0.1649 & 0.0018 & 1.614 & 0.022 & 0.0710 & 0.0006 & 0.816 & 984 & 10 & 957 & 16 & -3 \\
\hline
\end{tabular}

Notes: $\quad$ 1. Uncertainties given at the one $\sigma$ level.

2. Error in Temora reference zircon calibration was $0.89 \%$ for the analytical session. (not included in above errors but required when comparing ${ }^{200} \mathrm{~Pb} /{ }^{2 s s} \mathrm{U}$ data from different mounts).

3. $\mathrm{f}_{206} \%$ denotes the percentage of ${ }^{206} \mathrm{~Pb}$ that is common $\mathrm{Pb}$.

4. For areas older than $\sim 800 \mathrm{Ma}$ correction for common $\mathrm{Pb}$ made using the measured ${ }^{204} \mathrm{~Pb} /{ }^{206} \mathrm{~Pb}$ ratio.

5. For areas younger than $\sim 800 \mathrm{Ma}$ correction for common $\mathrm{Pb}$ made using the measured ${ }^{238} \mathrm{U} /{ }^{206} \mathrm{~Pb}$ and ${ }^{207} \mathrm{~Pb} /{ }^{206} \mathrm{~Pb}$ ratios following Tera and Wasserburg (1972) as outlined in Williams (1998).

6. For $\%$ Disc, $0 \%$ denotes a concordant analysis. 
Table 4. SHRIMP U-Pb data for zircon grains from sample PV.

\begin{tabular}{|c|c|c|c|c|c|c|c|c|c|c|c|c|c|c|c|c|c|c|c|c|c|c|}
\hline \multirow[b]{2}{*}{$\begin{array}{c}\text { Grain. } \\
\text { spot }\end{array}$} & \multirow[b]{2}{*}{$\underset{(\mathrm{ppm})}{\mathrm{U}}$} & \multirow[b]{2}{*}{$\begin{array}{c}\text { Th } \\
(\mathrm{ppm})\end{array}$} & \multirow[b]{2}{*}{$\mathrm{Th} / \mathrm{U}$} & & & & & Total F & atios & & & & adiogeni & Ratios & & & & & & e (Ma) & & \\
\hline & & & & $\begin{array}{l}{ }^{206} \mathrm{~Pb}^{*} \\
(\mathrm{ppm})\end{array}$ & $\begin{array}{l}{ }^{204} \mathrm{~Pb} / \\
{ }^{206} \mathrm{~Pb}\end{array}$ & $\begin{array}{l}f_{206} \\
\%\end{array}$ & $\begin{array}{l}{ }^{238} \mathrm{U} / \\
{ }^{206} \mathrm{~Pb}\end{array}$ & \pm & $\begin{array}{l}{ }^{207} \mathrm{~Pb} / \\
{ }^{206} \mathrm{~Pb}\end{array}$ & \pm & $\begin{array}{l}{ }^{206} \mathrm{~Pb} / \\
{ }^{238} \mathrm{U}\end{array}$ & \pm & $\begin{array}{l}{ }^{207} \mathrm{~Pb} / \\
{ }^{235} \mathrm{U}\end{array}$ & \pm & $\begin{array}{l}{ }^{207} \mathrm{~Pb} / \\
{ }^{206} \mathrm{~Pb}\end{array}$ & \pm & $\rho$ & ${ }^{206} \mathrm{~Pb} /$ & \pm & $\begin{array}{l}{ }^{207} \mathrm{~Pb} / \\
{ }^{206} \mathrm{~Pb}\end{array}$ & \pm & $\begin{array}{c}\% \\
\text { Disc }\end{array}$ \\
\hline 1.1 & 116 & 54 & 0.47 & 16 & - & $<0.01$ & 6.093 & 0.077 & 0.0735 & 0.0009 & 0.1644 & 0.0021 & 1.693 & 0.036 & 0.0747 & 0.0013 & 0.595 & 981 & 12 & 1061 & 34 & 8 \\
\hline 2.1 & 38 & 22 & 0.58 & 16 & 0.000185 & 0.26 & 2.115 & 0.032 & 0.1628 & 0.0015 & 0.4715 & 0.0072 & 10.433 & 0.203 & 0.1605 & 0.0019 & 0.782 & 2490 & 31 & 2461 & 20 & -1 \\
\hline 3.1 & 466 & 105 & 0.23 & 152 & 0.000027 & 0.04 & 2.629 & 0.028 & 0.2066 & 0.0006 & 0.3802 & 0.0041 & 10.812 & 0.120 & 0.2062 & 0.0006 & 0.965 & 2077 & 19 & 2876 & 5 & 28 \\
\hline 4.1 & 285 & 126 & 0.44 & 79 & 0.000026 & 0.04 & 3.100 & 0.034 & 0.1218 & 0.0010 & 0.3224 & 0.0035 & 5.401 & 0.074 & 0.1215 & 0.0010 & 0.804 & 1802 & 17 & 1978 & 14 & 9 \\
\hline 5.1 & 1940 & 445 & 0.23 & 122 & 0.000188 & 0.34 & 13.625 & 0.139 & 0.0682 & 0.0003 & 0.0731 & 0.0007 & & & & & & 455 & 4 & & & \\
\hline 6.1 & 26 & 36 & 1.35 & 4 & 0.000888 & 1.50 & 5.497 & 0.101 & 0.0739 & 0.0018 & 0.1806 & 0.0043 & 1.688 & 0.211 & 0.0678 & 0.0072 & 0.811 & 1070 & 23 & 862 & 221 & -24 \\
\hline 7.1 & 140 & 95 & 0.68 & 50 & 0.000059 & 0.09 & 2.398 & 0.028 & 0.1636 & 0.0021 & 0.4166 & 0.0048 & 9.357 & 0.165 & 0.1629 & 0.0021 & 0.662 & 2245 & 22 & 2486 & 22 & 10 \\
\hline 8.1 & 80 & 38 & 0.48 & 12 & 0.000089 & 0.15 & 5.507 & 0.073 & 0.0765 & 0.0010 & 0.1813 & 0.0024 & 1.882 & 0.048 & 0.0753 & 0.0016 & 0.528 & 1074 & 13 & 1075 & 43 & 0 \\
\hline 9.1 & 1294 & 329 & 0.25 & 104 & 0.000177 & 0.31 & 10.662 & 0.110 & 0.0697 & 0.0006 & 0.0935 & 0.0010 & & & & & & 576 & 6 & & & \\
\hline 10.1 & 42 & 19 & 0.44 & 6 & 0.000118 & 0.20 & 5.986 & 0.092 & 0.0743 & 0.0015 & 0.1667 & 0.0026 & 1.669 & 0.057 & 0.0726 & 0.0022 & 0.453 & 994 & 14 & 1003 & 62 & 1 \\
\hline 11.1 & 321 & 89 & 0.28 & 71 & - & $<0.01$ & 3.854 & 0.042 & 0.0961 & 0.0022 & 0.2595 & 0.0028 & 3.441 & 0.086 & 0.0962 & 0.0022 & 0.431 & 1487 & 14 & 1551 & 42 & 4 \\
\hline 12.1 & 531 & 166 & 0.31 & 88 & 0.000011 & 0.02 & 5.195 & 0.055 & 0.0783 & 0.0004 & 0.1925 & 0.0020 & 2.075 & 0.025 & 0.0782 & 0.0004 & 0.883 & 1135 & 11 & 1151 & 11 & 1 \\
\hline 13.1 & 427 & 139 & 0.32 & 70 & 0.000013 & 0.02 & 5.245 & 0.056 & 0.0759 & 0.0004 & 0.1913 & 0.0021 & 2.073 & 0.041 & 0.0786 & 0.0009 & 0.843 & 1128 & 12 & 1163 & 24 & 3 \\
\hline 14.1 & 90 & 32 & 0.36 & 12 & - & $<0.01$ & 6.498 & 0.086 & 0.0723 & 0.0011 & 0.1534 & 0.0021 & 1.475 & 0.050 & 0.0697 & 0.0018 & 0.731 & 920 & 12 & 921 & 52 & 0 \\
\hline 15.1 & 235 & 135 & 0.57 & 59 & - & $<0.01$ & 3.405 & 0.038 & 0.1056 & 0.0006 & 0.2937 & 0.0032 & 4.288 & 0.053 & 0.1059 & 0.0006 & 0.886 & 1660 & 16 & 1730 & 11 & 4 \\
\hline 16.1 & 34 & 26 & 0.78 & 13 & - & $<0.01$ & 2.178 & 0.033 & 0.1642 & 0.0016 & 0.4591 & 0.0069 & 10.398 & 0.184 & 0.1642 & 0.0016 & 0.845 & 2436 & 30 & 2500 & 16 & 3 \\
\hline 17.1 & 918 & 230 & 0.25 & 86 & 0.000039 & 0.07 & 9.216 & 0.096 & 0.0623 & 0.0004 & 0.1084 & 0.0011 & & & & & & 664 & 7 & & & \\
\hline 18.1 & 126 & 131 & 1.04 & 18 & 0.000025 & 0.04 & 5.882 & 0.071 & 0.0730 & 0.0008 & 0.1699 & 0.0021 & 1.702 & 0.029 & 0.0726 & 0.0009 & 0.712 & 1012 & 11 & 1004 & 24 & -1 \\
\hline 19.1 & 113 & 118 & 1.04 & 16 & 0.000109 & 0.19 & 6.168 & 0.076 & 0.0710 & 0.0009 & 0.1624 & 0.0024 & 1.621 & 0.089 & 0.0724 & 0.0032 & 0.818 & 970 & 13 & 997 & 89 & 3 \\
\hline 20.1 & 352 & 423 & 1.20 & 88 & 0.000077 & 0.12 & 3.455 & 0.037 & 0.1097 & 0.0005 & 0.2891 & 0.0031 & 4.330 & 0.051 & 0.1086 & 0.0005 & 0.913 & 1637 & 15 & 1777 & 9 & 8 \\
\hline 21.1 & 227 & 72 & 0.32 & 140 & 0.000005 & 0.01 & 1.397 & 0.015 & 0.2986 & 0.0014 & 0.7156 & 0.0078 & 29.459 & 0.349 & 0.2986 & 0.0014 & 0.918 & 3480 & 29 & 3463 & 7 & 0 \\
\hline 22.1 & 262 & 44 & 0.17 & 38 & 0.000064 & 0.11 & 5.846 & 0.064 & 0.0705 & 0.0005 & 0.1712 & 0.0019 & 1.677 & 0.030 & 0.0711 & 0.0007 & 0.827 & 1019 & 11 & 959 & 21 & -6 \\
\hline 23.1 & 275 & 114 & 0.41 & 40 & 0.000018 & 0.03 & 5.920 & 0.065 & 0.0726 & 0.0005 & 0.1689 & 0.0019 & 1.685 & 0.023 & 0.0724 & 0.0006 & 0.799 & 1006 & 10 & 997 & 17 & -1 \\
\hline 24.1 & 326 & 40 & 0.12 & 68 & 0.000010 & 0.02 & 4.112 & 0.044 & 0.1208 & 0.0011 & 0.2432 & 0.0026 & 4.046 & 0.057 & 0.1207 & 0.0011 & 0.765 & 1403 & 14 & 1966 & 16 & 29 \\
\hline 25.1 & 424 & 159 & 0.37 & 50 & 0.000075 & 0.13 & 7.310 & 0.078 & 0.0664 & 0.0004 & 0.1366 & 0.0015 & 1.231 & 0.017 & 0.0653 & 0.0006 & 0.773 & 825 & 8 & 785 & 18 & -5 \\
\hline 26.1 & 56 & 29 & 0.52 & 8 & - & $<0.01$ & 5.664 & 0.082 & 0.0808 & 0.0013 & 0.1772 & 0.0026 & 2.044 & 0.057 & 0.0837 & 0.0020 & 0.521 & 1052 & 14 & 1285 & 46 & 18 \\
\hline 27.1 & 206 & 71 & 0.34 & 32 & 0.000003 & 0.00 & 5.492 & 0.062 & 0.0745 & 0.0006 & 0.1821 & 0.0020 & 1.869 & 0.026 & 0.0745 & 0.0006 & 0.820 & 1078 & 11 & 1054 & 16 & -2 \\
\hline 28.1 & 661 & 439 & 0.66 & 165 & 0.000005 & 0.01 & 3.448 & 0.036 & 0.1033 & 0.0003 & 0.2900 & 0.0030 & 4.127 & 0.045 & 0.1032 & 0.0003 & 0.958 & 1642 & 15 & 1682 & 6 & 2 \\
\hline 29.1 & 878 & 35 & 0.04 & 116 & 0.000068 & 0.12 & 6.517 & 0.067 & 0.0702 & 0.0003 & 0.1533 & 0.0016 & 1.462 & 0.017 & 0.0692 & 0.0004 & 0.887 & 919 & 9 & 905 & 11 & -2 \\
\hline 30.1 & 241 & 166 & 0.69 & 85 & 0.000044 & 0.06 & 2.422 & 0.026 & 0.1665 & 0.0006 & 0.4127 & 0.0045 & 9.441 & 0.108 & 0.1659 & 0.0006 & 0.949 & 2227 & 20 & 2517 & 6 & 12 \\
\hline 31.1 & 221 & 722 & 3.27 & 42 & 0.000021 & 0.03 & 4.475 & 0.049 & 0.0841 & 0.0006 & 0.2234 & 0.0025 & 2.583 & 0.034 & 0.0839 & 0.0006 & 0.831 & 1300 & 13 & 1289 & 14 & -1 \\
\hline 32.1 & 48 & 81 & 1.69 & 15 & 0.000129 & 0.20 & 2.686 & 0.037 & 0.1471 & 0.0030 & 0.3716 & 0.0052 & 7.452 & 0.191 & 0.1454 & 0.0031 & 0.542 & 2037 & 24 & 2293 & 37 & 11 \\
\hline 33.1 & 115 & 110 & 0.96 & 13 & - & $<0.01$ & 7.512 & 0.092 & 0.0665 & 0.0009 & 0.1331 & 0.0016 & 1.222 & 0.022 & 0.0666 & 0.0009 & 0.683 & 806 & 9 & 824 & 27 & 2 \\
\hline 34.1 & 1692 & 51 & 0.03 & 527 & 0.000025 & 0.04 & 2.756 & 0.028 & 0.1777 & 0.0002 & 0.3627 & 0.0037 & 8.869 & 0.091 & 0.1774 & 0.0003 & 0.990 & 1995 & 17 & 2628 & 2 & 24 \\
\hline 35.1 & 1032 & 38 & 0.04 & 287 & 0.000015 & 0.02 & 3.091 & 0.031 & 0.1390 & 0.0009 & 0.3235 & 0.0033 & 6.189 & 0.075 & 0.1388 & 0.0009 & 0.846 & 1807 & 16 & 2212 & 11 & 18 \\
\hline 36.1 & 275 & 157 & 0.57 & 40 & 0.000004 & 0.01 & 5.969 & 0.065 & 0.0711 & 0.0005 & 0.1675 & 0.0018 & 1.641 & 0.021 & 0.0711 & 0.0005 & 0.837 & 998 & 10 & 959 & 14 & -4 \\
\hline 37.1 & 334 & 179 & 0.54 & 127 & 0.000003 & 0.00 & 2.262 & 0.024 & 0.1513 & 0.0004 & 0.4421 & 0.0047 & 9.223 & 0.101 & 0.1513 & 0.0004 & 0.964 & 2360 & 21 & 2361 & 5 & 0 \\
\hline 38.1 & 254 & 196 & 0.77 & 36 & 0.000019 & 0.03 & 6.077 & 0.068 & 0.0727 & 0.0006 & 0.1645 & 0.0018 & 1.643 & 0.024 & 0.0724 & 0.0007 & 0.776 & 982 & 10 & 999 & 18 & 2 \\
\hline 39.1 & 198 & 87 & 0.44 & 31 & 0.000105 & 0.18 & 5.574 & 0.067 & 0.0754 & 0.0007 & 0.1791 & 0.0022 & 1.826 & 0.036 & 0.0739 & 0.0011 & 0.613 & 1062 & 12 & 1040 & 31 & -2 \\
\hline 40.1 & 291 & 54 & 0.19 & 39 & 0.000101 & 0.17 & 6.362 & 0.071 & 0.0704 & 0.0006 & 0.1572 & 0.0018 & 1.530 & 0.054 & 0.0706 & 0.0021 & 0.594 & 941 & 10 & 946 & 61 & 0 \\
\hline 41.1 & 143 & 117 & 0.82 & 21 & 0.000067 & 0.11 & 5.889 & 0.070 & 0.0761 & 0.0008 & 0.1696 & 0.0020 & 1.757 & 0.036 & 0.0751 & 0.0012 & 0.590 & 1010 & 11 & 1072 & 33 & 6 \\
\hline 42.1 & 377 & 9 & 0.02 & 53 & 0.000016 & 0.03 & 6.132 & 0.066 & 0.0698 & 0.0005 & 0.1630 & 0.0018 & 1.564 & 0.020 & 0.0696 & 0.0005 & 0.825 & 974 & 10 & 916 & 15 & -6 \\
\hline 43.1 & 690 & 16 & 0.02 & 91 & 0.000033 & 0.06 & 6.505 & 0.068 & 0.0691 & 0.0004 & 0.1536 & 0.0016 & 1.455 & 0.018 & 0.0687 & 0.0005 & 0.846 & 921 & 9 & 889 & 14 & -4 \\
\hline 44.1 & 69 & 24 & 0.36 & 20 & 0.000017 & 0.03 & 2.994 & 0.041 & 0.1608 & 0.0012 & 0.3339 & 0.0046 & 7.395 & 0.116 & 0.1606 & 0.0013 & 0.867 & 1857 & 22 & 2462 & 13 & 25 \\
\hline 45.1 & 103 & 115 & 1.12 & 9 & 0.000172 & 0.31 & 10.191 & 0.145 & 0.0586 & 0.0011 & 0.0978 & 0.0014 & & & & & & 602 & 8 & & & \\
\hline 46.1 & 729 & 285 & 0.39 & 68 & 0.000028 & 0.05 & 9.234 & 0.097 & 0.0624 & 0.0004 & 0.1082 & 0.0011 & & & & & & 663 & 7 & & & \\
\hline 47.1 & 808 & 819 & 1.01 & 63 & 0.000036 & 0.06 & 11.092 & 0.116 & 0.0592 & 0.0004 & 0.0901 & 0.0009 & & & & & & 556 & 6 & & & \\
\hline 48.1 & 129 & 63 & 0.48 & 29 & 0.000026 & 0.04 & 3.880 & 0.046 & 0.0919 & 0.0008 & 0.2576 & 0.0031 & 3.251 & 0.051 & 0.0915 & 0.0009 & 0.768 & 1478 & 16 & 1457 & 19 & -1 \\
\hline 49.1 & 900 & 11 & 0.01 & 126 & 0.000026 & 0.05 & 6.113 & 0.063 & 0.0714 & 0.0003 & 0.1635 & 0.0017 & 1.600 & 0.019 & 0.0710 & 0.0004 & 0.894 & 976 & 9 & 957 & 11 & -2 \\
\hline 50.1 & 670 & 125 & 0.19 & 90 & 0.000010 & 0.02 & 6.422 & 0.067 & 0.0694 & 0.0004 & 0.1557 & 0.0016 & 1.488 & 0.018 & 0.0693 & 0.0004 & 0.882 & 933 & 9 & 908 & 12 & -3 \\
\hline 51.1 & 376 & 130 & 0.35 & 60 & - & $<0.01$ & 5.346 & 0.058 & 0.0753 & 0.0005 & 0.1871 & 0.0020 & 1.942 & 0.024 & 0.0753 & 0.0005 & 0.865 & 1105 & 11 & 1076 & 13 & -3 \\
\hline 52.1 & 39 & 13 & 0.32 & 17 & 0.000045 & 0.06 & 1.971 & 0.029 & 0.1734 & 0.0015 & 0.5071 & 0.0075 & 12.084 & 0.210 & 0.1728 & 0.0016 & 0.852 & 2644 & 32 & 2585 & 15 & -2 \\
\hline 53.1 & 270 & 177 & 0.66 & 40 & - & $<0.01$ & 5.827 & 0.065 & 0.0726 & 0.0006 & 0.1716 & 0.0019 & 1.719 & 0.023 & 0.0726 & 0.0006 & 0.821 & 1021 & 10 & 1004 & 16 & -2 \\
\hline 54.1 & 63 & 35 & 0.55 & 15 & 0.000033 & 0.05 & 3.473 & 0.047 & 0.1000 & 0.0011 & 0.2878 & 0.0039 & 3.952 & 0.070 & 0.0996 & 0.0011 & 0.760 & 1631 & 19 & 1616 & 21 & -1 \\
\hline 55.1 & 94 & 70 & 0.75 & 13 & 0.000050 & 0.09 & 6.025 & 0.077 & 0.0726 & 0.0010 & 0.1658 & 0.0021 & 1.643 & 0.034 & 0.0719 & 0.0012 & 0.616 & 989 & 12 & 982 & 34 & -1 \\
\hline 56.1 & 205 & 127 & 0.62 & 34 & 0.000123 & 0.21 & 5.118 & 0.058 & 0.0768 & 0.0013 & 0.1962 & 0.0024 & 2.169 & 0.077 & 0.0802 & 0.0022 & 0.724 & 1155 & 13 & 1201 & 55 & 4 \\
\hline 57.1 & 48 & 35 & 0.73 & 7 & 0.000189 & 0.32 & 6.229 & 0.095 & 0.0726 & 0.0014 & 0.1600 & 0.0025 & 1.542 & 0.070 & 0.0699 & 0.0030 & 0.340 & 957 & 14 & 925 & 88 & -3 \\
\hline 58.1 & 1520 & 146 & 0.10 & 578 & 0.000006 & 0.01 & 2.260 & 0.023 & 0.1615 & 0.0021 & 0.4425 & 0.0045 & 9.848 & 0.161 & 0.1614 & 0.0021 & 0.623 & 2362 & 20 & 2470 & 22 & 4 \\
\hline 59.1 & 178 & 72 & 0.40 & 45 & 0.000030 & 0.05 & 3.372 & 0.038 & 0.1050 & 0.0006 & 0.2964 & 0.0034 & 4.273 & 0.058 & 0.1046 & 0.0008 & 0.845 & 1674 & 17 & 1706 & 13 & 2 \\
\hline 60.1 & 1376 & 313 & 0.23 & 106 & 0.000103 & 0.18 & 11.166 & 0.115 & 0.0618 & 0.0003 & 0.0894 & 0.0009 & & & & & & 552 & 5 & & & \\
\hline 61.1 & 33 & 44 & 1.34 & 4 & 0.000957 & 1.66 & 7.147 & 0.122 & 0.0675 & 0.0017 & 0.1383 & 0.0031 & 1.100 & 0.155 & 0.0577 & 0.0071 & 0.805 & 835 & 18 & 519 & 271 & -61 \\
\hline 62.1 & 261 & 110 & 0.42 & 28 & - & $<0.01$ & 8.044 & 0.091 & 0.0637 & 0.0006 & 0.1244 & 0.0014 & & & & & & 756 & 8 & & & \\
\hline 63.1 & 906 & 40 & 0.04 & 369 & - & $<0.01$ & 2.112 & 0.022 & 0.1494 & 0.0026 & 0.4735 & 0.0049 & 9.753 & 0.197 & 0.1494 & 0.0026 & 0.508 & 2499 & 21 & 2339 & 30 & -7 \\
\hline 64.1 & 140 & 83 & 0.59 & 70 & 0.000032 & 0.04 & 1.714 & 0.020 & 0.2188 & 0.0008 & 0.5832 & 0.0067 & 17.562 & 0.213 & 0.2184 & 0.0008 & 0.951 & 2962 & 27 & 2969 & 6 & 0 \\
\hline 65.1 & 285 & 158 & 0.56 & 30 & 0.000051 & 0.09 & 8.184 & 0.091 & 0.0844 & 0.0069 & 0.1221 & 0.0014 & & & & & & 742 & 8 & & & \\
\hline
\end{tabular}

Notes : $\quad$ 1. Uncertainties given at the one $\sigma$ level.

2. Error in Temora reference zircon calibration was $0.80 \%$ for the analytical session.

( not included in above errors but required when comparing ${ }^{206} \mathrm{~Pb}^{238} \mathrm{U}$ data from different mounts)

3. $\mathrm{f}_{206} \%$ denotes the percentage of ${ }^{206} \mathrm{~Pb}$ that is common $\mathrm{Pb}$.

4. Correction for common $\mathrm{Pb}$ made using the measured ${ }^{204} \mathrm{~Pb} / 206 \mathrm{~Pb}$ ratio.

5. For $\%$ Disc, $0 \%$ denotes a concordant analysis. 
Table 5. SHRIMP U-Pb data for zircon grains from sample Tal-GKM.

\begin{tabular}{|c|c|c|c|c|c|c|c|c|c|c|c|c|c|c|c|c|c|c|c|c|c|c|}
\hline \multirow[b]{2}{*}{$\begin{array}{c}\text { Grain. } \\
\text { spot }\end{array}$} & \multirow[b]{2}{*}{$\underset{(\mathrm{ppm})}{\mathrm{U}}$} & \multirow[b]{2}{*}{$\begin{array}{c}\text { Th } \\
\text { (ppm) }\end{array}$} & \multirow[b]{2}{*}{$\mathrm{Th} / \mathrm{U}$} & & & & & Total R & atios & & & & adiogeni & Ratios & & & & & & (Ma) & & \\
\hline & & & & $\begin{array}{l}{ }^{206} \mathrm{~Pb}^{\star} \\
(\mathrm{ppm})\end{array}$ & $\begin{array}{l}{ }^{204} \mathrm{~Pb} / \\
{ }^{206} \mathrm{~Pb}\end{array}$ & $\begin{array}{l}f_{206} \\
\%\end{array}$ & $\begin{array}{l}{ }^{238} \mathrm{U} / \\
{ }^{206} \mathrm{~Pb}\end{array}$ & \pm & $\begin{array}{l}{ }^{207} \mathrm{~Pb} / \\
{ }^{006} \mathrm{~Pb}\end{array}$ & \pm & $\begin{array}{l}{ }^{206} \mathrm{~Pb} / \\
{ }^{238} \mathrm{U}\end{array}$ & \pm & $\begin{array}{c}{ }^{207} \mathrm{~Pb} / \\
{ }^{235} \mathrm{U}\end{array}$ & \pm & $\begin{array}{l}{ }^{207} \mathrm{~Pb} / \\
{ }^{206} \mathrm{~Pb}\end{array}$ & \pm & $\rho$ & $\begin{array}{l}{ }^{206} \mathrm{~Pb} / \\
{ }^{238} \mathrm{U}\end{array}$ & \pm & $\begin{array}{l}{ }^{207} \mathrm{~Pb} / \\
{ }^{206} \mathrm{~Pb}\end{array}$ & \pm & $\begin{array}{c}\% \\
\text { Disc }\end{array}$ \\
\hline 1.1 & 105 & 38 & 0.36 & 15 & 0.000025 & 0.04 & 6.112 & 0.095 & 0.0702 & 0.0013 & 0.1635 & 0.0025 & 1.574 & 0.039 & 0.0698 & 0.0013 & 0.626 & 976 & 14 & 923 & 40 & -6 \\
\hline 2.1 & 290 & 215 & 0.74 & 118 & 0.000031 & 0.04 & 2.114 & 0.025 & 0.1644 & 0.0008 & 0.4728 & 0.0056 & 10.692 & 0.137 & 0.1640 & 0.0008 & 0.926 & 2496 & 25 & 2498 & 8 & 0 \\
\hline 3.1 & 430 & 255 & 0.59 & 33 & 0.000094 & $<0.01$ & 11.178 & 0.135 & 0.0582 & 0.0008 & 0.0895 & 0.0011 & & & & & & 553 & 7 & & & \\
\hline 4.1 & 99 & 79 & 0.80 & 11 & 0.000205 & 0.36 & 7.436 & 0.121 & 0.0652 & 0.0015 & 0.1345 & 0.0025 & 1.214 & 0.087 & 0.0654 & 0.0038 & 0.752 & 814 & 14 & 789 & 123 & -3 \\
\hline 5.1 & 42 & 36 & 0.86 & 5 & 0.000161 & 0.28 & 7.320 & 0.161 & 0.0677 & 0.0022 & 0.1367 & 0.0035 & 1.280 & 0.134 & 0.0679 & 0.0059 & 0.748 & 826 & 20 & 866 & 181 & 5 \\
\hline 6.1 & 158 & 182 & 1.15 & 18 & 0.000303 & 0.54 & 7.550 & 0.108 & 0.0652 & 0.0012 & 0.1328 & 0.0023 & 1.235 & 0.096 & 0.0674 & 0.0043 & 0.808 & 804 & 13 & 852 & 133 & 6 \\
\hline 7.1 & 322 & 287 & 0.89 & 136 & - & $<0.01$ & 2.034 & 0.024 & 0.1654 & 0.0007 & 0.4916 & 0.0058 & 11.209 & 0.141 & 0.1654 & 0.0007 & 0.937 & 2577 & 25 & 2512 & 7 & -3 \\
\hline 8.1 & 974 & 369 & 0.38 & 244 & 0.000299 & 0.43 & 3.423 & 0.037 & 0.1616 & 0.0005 & 0.2909 & 0.0031 & 6.329 & 0.073 & 0.1578 & 0.0007 & 0.933 & 1646 & 16 & 2432 & 7 & 32 \\
\hline 9.1 & 165 & 153 & 0.93 & 59 & - & $<0.01$ & 2.390 & 0.032 & 0.1375 & 0.0010 & 0.4184 & 0.0056 & 7.933 & 0.121 & 0.1375 & 0.0010 & 0.879 & 2253 & 25 & 2196 & 13 & -3 \\
\hline 10.1 & 62 & 45 & 0.73 & 9 & 0.000358 & 0.62 & 6.168 & 0.116 & 0.0730 & 0.0018 & 0.1611 & 0.0031 & 1.508 & 0.092 & 0.0679 & 0.0039 & 0.317 & 963 & 17 & 865 & 119 & -11 \\
\hline 11.1 & 160 & 116 & 0.72 & 67 & 0.000028 & 0.04 & 2.069 & 0.028 & 0.1667 & 0.0011 & 0.4832 & 0.0065 & 11.086 & 0.165 & 0.1664 & 0.0011 & 0.901 & 2541 & 28 & 2522 & 11 & -1 \\
\hline 12.1 & 331 & 214 & 0.65 & 40 & - & $<0.01$ & 7.147 & 0.088 & 0.0669 & 0.0008 & 0.1399 & 0.0017 & 1.292 & 0.022 & 0.0670 & 0.0008 & 0.713 & 844 & 10 & 837 & 25 & -1 \\
\hline 13.1 & 31 & 41 & 1.35 & 2 & 0.001070 & 0.64 & 10.885 & 0.297 & 0.0641 & 0.0032 & 0.0913 & 0.0026 & & & & & & 563 & 15 & & & \\
\hline 14.1 & 400 & 30 & 0.07 & 38 & 0.000055 & 0.01 & 9.075 & 0.109 & 0.0621 & 0.0008 & 0.1102 & 0.0014 & & & & & & 674 & 8 & & & \\
\hline 15.1 & 217 & 83 & 0.38 & 30 & 0.000046 & 0.08 & 6.113 & 0.081 & 0.0730 & 0.0010 & 0.1635 & 0.0022 & 1.630 & 0.032 & 0.0723 & 0.0010 & 0.684 & 976 & 12 & 995 & 29 & 2 \\
\hline 16.1 & 94 & 84 & 0.89 & 13 & 0.000173 & 0.30 & 6.240 & 0.103 & 0.0692 & 0.0014 & 0.1603 & 0.0031 & 1.529 & 0.113 & 0.0692 & 0.0042 & 0.775 & 958 & 17 & 905 & 124 & -6 \\
\hline 17.1 & 529 & 489 & 0.93 & 56 & 0.000119 & 0.11 & 8.056 & 0.093 & 0.0653 & 0.0007 & 0.1240 & 0.0015 & & & & & & 753 & 8 & & & \\
\hline 18.1 & 274 & 183 & 0.67 & 45 & 0.000075 & 0.13 & 5.170 & 0.065 & 0.0784 & 0.0008 & 0.1932 & 0.0024 & 2.059 & 0.036 & 0.0773 & 0.0009 & 0.720 & 1139 & 13 & 1129 & 24 & -1 \\
\hline 19.1 & 116 & 37 & 0.32 & 16 & 0.000063 & 0.11 & 6.334 & 0.098 & 0.0704 & 0.0013 & 0.1577 & 0.0025 & 1.512 & 0.040 & 0.0695 & 0.0015 & 0.587 & 944 & 14 & 914 & 44 & -3 \\
\hline 20.1 & 701 & 277 & 0.40 & 21 & 0.000024 & 2.52 & 28.848 & 0.344 & 0.0705 & 0.0005 & 0.0338 & 0.0004 & & & & & & 214 & 3 & & & \\
\hline 21.1 & 149 & 93 & 0.62 & 16 & 0.000069 & 0.12 & 8.080 & 0.119 & 0.0629 & 0.0012 & 0.1236 & 0.0018 & 1.056 & 0.028 & 0.0619 & 0.0013 & 0.563 & 751 & 10 & 672 & 46 & -12 \\
\hline 22.1 & 263 & 87 & 0.33 & 67 & 0.000052 & 0.08 & 3.392 & 0.042 & 0.1122 & 0.0027 & 0.2946 & 0.0036 & 4.530 & 0.125 & 0.1115 & 0.0028 & 0.447 & 1664 & 18 & 1825 & 45 & 9 \\
\hline 23.1 & 162 & 80 & 0.50 & 19 & 0.000072 & 0.13 & 7.118 & 0.101 & 0.0665 & 0.0011 & 0.1410 & 0.0022 & 1.351 & 0.055 & 0.0695 & 0.0022 & 0.743 & 850 & 12 & 914 & 64 & 7 \\
\hline 24.1 & 653 & 39 & 0.06 & 57 & 0.000137 & 0.24 & 9.873 & 0.112 & 0.0624 & 0.0006 & 0.1010 & 0.0012 & & & & & & 621 & 7 & & & \\
\hline 25.1 & 172 & 196 & 1.14 & 23 & 0.000073 & 0.13 & 6.346 & 0.088 & 0.0709 & 0.0011 & 0.1574 & 0.0022 & 1.516 & 0.034 & 0.0699 & 0.0012 & 0.621 & 942 & 12 & 925 & 36 & -2 \\
\hline 26.1 & 774 & 53 & 0.07 & 75 & 0.000031 & $<0.01$ & 8.840 & 0.098 & 0.0624 & 0.0006 & 0.1131 & 0.0013 & & & & & & 691 & 7 & & & \\
\hline 27.1 & 223 & 41 & 0.18 & 26 & 0.000084 & 0.15 & 7.389 & 0.098 & 0.0639 & 0.0010 & 0.1356 & 0.0019 & 1.224 & 0.032 & 0.0655 & 0.0012 & 0.710 & 820 & 11 & 790 & 40 & -4 \\
\hline 28.1 & 218 & 118 & 0.54 & 18 & 0.000358 & $<0.01$ & 10.330 & 0.141 & 0.0579 & 0.0011 & 0.0970 & 0.0014 & & & & & & 597 & 8 & & & \\
\hline 29.1 & 230 & 177 & 0.77 & 17 & 0.000205 & 0.48 & 11.571 & 0.160 & 0.0619 & 0.0012 & 0.0860 & 0.0012 & & & & & & 532 & 7 & & & \\
\hline 30.1 & 330 & 108 & 0.33 & 31 & 0.000124 & 0.03 & 9.224 & 0.115 & 0.0619 & 0.0009 & 0.1084 & 0.0014 & & & & & & 663 & 8 & & & \\
\hline 32.1 & 105 & 115 & 1.10 & 8 & 0.000422 & 0.25 & 11.266 & 0.196 & 0.0605 & 0.0018 & 0.0885 & 0.0016 & & & & & & 547 & 9 & & & \\
\hline 33.1 & 484 & 403 & 0.83 & 36 & 0.000052 & 0.05 & 11.402 & 0.136 & 0.0588 & 0.0010 & 0.0877 & 0.0011 & & & & & & 542 & 6 & & & \\
\hline 34.1 & 75 & 141 & 1.87 & 6 & 0.000178 & 0.52 & 10.272 & 0.196 & 0.0640 & 0.0020 & 0.0968 & 0.0019 & & & & & & 596 & 11 & & & \\
\hline 35.1 & 202 & 59 & 0.29 & 25 & 0.000106 & 0.18 & 6.918 & 0.094 & 0.0704 & 0.0010 & 0.1443 & 0.0020 & 1.370 & 0.037 & 0.0689 & 0.0016 & 0.501 & 869 & 11 & 895 & 49 & 3 \\
\hline 36.1 & 469 & 791 & 1.69 & 53 & 0.000024 & 0.04 & 7.568 & 0.089 & 0.0655 & 0.0007 & 0.1321 & 0.0016 & 1.186 & 0.019 & 0.0651 & 0.0007 & 0.732 & 800 & 9 & 779 & 23 & -3 \\
\hline 37.1 & 279 & 328 & 1.17 & 31 & - & $<0.01$ & 7.662 & 0.098 & 0.0664 & 0.0009 & 0.1305 & 0.0017 & 1.195 & 0.022 & 0.0664 & 0.0009 & 0.686 & 791 & 10 & 819 & 28 & 3 \\
\hline 38.1 & 323 & 135 & 0.42 & 38 & 0.000079 & 0.14 & 7.261 & 0.090 & 0.0695 & 0.0008 & 0.1375 & 0.0017 & 1.297 & 0.028 & 0.0684 & 0.0012 & 0.582 & 831 & 10 & 880 & 36 & 6 \\
\hline 39.1 & 181 & 710 & 3.92 & 14 & 0.000080 & 0.17 & 11.246 & 0.164 & 0.0598 & 0.0013 & 0.0888 & 0.0013 & & & & & & 548 & 8 & & & \\
\hline 40.1 & 87 & 72 & 0.82 & 20 & 0.000151 & 0.24 & 3.752 & 0.061 & 0.0978 & 0.0014 & 0.2659 & 0.0043 & 3.509 & 0.089 & 0.0957 & 0.0019 & 0.639 & 1520 & 22 & 1543 & 37 & 1 \\
\hline 41.1 & 236 & 92 & 0.39 & 96 & - & $<0.01$ & 2.106 & 0.026 & 0.1726 & 0.0009 & 0.4749 & 0.0059 & 11.302 & 0.152 & 0.1726 & 0.0009 & 0.924 & 2505 & 26 & 2583 & 9 & 3 \\
\hline 42.1 & 188 & 96 & 0.51 & 18 & 0.000111 & 0.24 & 8.920 & 0.125 & 0.0642 & 0.0012 & 0.1118 & 0.0016 & & & & & & 683 & 9 & & & \\
\hline 43.1 & 91 & 176 & 1.94 & 10 & 0.000155 & 0.27 & 7.728 & 0.132 & 0.0677 & 0.0016 & 0.1291 & 0.0022 & 1.164 & 0.045 & 0.0654 & 0.0023 & 0.447 & 782 & 13 & 788 & 72 & 1 \\
\hline 44.1 & 377 & 153 & 0.41 & 40 & 0.000090 & $<0.01$ & 8.095 & 0.098 & 0.0630 & 0.0008 & 0.1237 & 0.0015 & & & & & & 752 & 9 & & & \\
\hline 45.1 & 92 & 144 & 1.56 & 7 & 0.000279 & 0.23 & 11.024 & 0.198 & 0.0606 & 0.0018 & 0.0905 & 0.0017 & & & & & & 559 & 10 & & & \\
\hline 46.1 & 575 & 174 & 0.30 & 88 & 0.000072 & 0.12 & 5.634 & 0.064 & 0.0744 & 0.0006 & 0.1776 & 0.0021 & 1.835 & 0.040 & 0.0749 & 0.0010 & 0.836 & 1054 & 12 & 1067 & 27 & 1 \\
\hline 47.1 & 152 & 48 & 0.32 & 22 & 0.000160 & 0.28 & 5.995 & 0.088 & 0.0718 & 0.0012 & 0.1670 & 0.0026 & 1.679 & 0.056 & 0.0729 & 0.0018 & 0.733 & 996 & 14 & 1012 & 50 & 2 \\
\hline 48.1 & 113 & 51 & 0.45 & 38 & 0.000014 & 0.02 & 2.559 & 0.039 & 0.1361 & 0.0013 & 0.3906 & 0.0060 & 7.321 & 0.131 & 0.1359 & 0.0013 & 0.852 & 2126 & 28 & 2176 & 16 & 2 \\
\hline 49.1 & 124 & 1 & 0.01 & 10 & 0.000379 & $<0.01$ & 10.417 & 0.174 & 0.0588 & 0.0016 & 0.0961 & 0.0016 & & & & & & 592 & 10 & & & \\
\hline 50.1 & 483 & 45 & 0.09 & 67 & - & $<0.01$ & 6.205 & 0.073 & 0.0727 & 0.0007 & 0.1612 & 0.0019 & 1.617 & 0.024 & 0.0728 & 0.0007 & 0.784 & 963 & 11 & 1007 & 19 & 4 \\
\hline 51.1 & 355 & 266 & 0.75 & 56 & 0.000149 & 0.25 & 5.461 & 0.067 & 0.0772 & 0.0008 & 0.1826 & 0.0022 & 1.890 & 0.033 & 0.0751 & 0.0009 & 0.697 & 1081 & 12 & 1070 & 25 & -1 \\
\hline 52.1 & 149 & 71 & 0.48 & 22 & 0.000162 & 0.28 & 5.936 & 0.089 & 0.0714 & 0.0012 & 0.1687 & 0.0027 & 1.681 & 0.072 & 0.0723 & 0.0024 & 0.734 & 1005 & 15 & 994 & 67 & -1 \\
\hline 53.1 & 76 & 88 & 1.15 & 31 & 0.000127 & 0.18 & 2.114 & 0.036 & 0.1698 & 0.0027 & 0.4721 & 0.0081 & 10.951 & 0.263 & 0.1682 & 0.0028 & 0.714 & 2493 & 35 & 2540 & 28 & 2 \\
\hline 54.1 & 94 & 38 & 0.40 & 12 & 0.000430 & 0.76 & 6.916 & 0.120 & 0.0668 & 0.0017 & 0.1448 & 0.0027 & 1.352 & 0.068 & 0.0678 & 0.0027 & 0.688 & 872 & 15 & 861 & 82 & -1 \\
\hline 55.1 & 275 & 77 & 0.28 & 25 & 0.000267 & 0.48 & 9.514 & 0.127 & 0.0650 & 0.0010 & 0.1046 & 0.0014 & & & & & & 641 & 8 & & & \\
\hline 56.1 & 463 & 122 & 0.26 & 60 & 0.000030 & 0.05 & 6.604 & 0.078 & 0.0705 & 0.0009 & 0.1513 & 0.0018 & 1.462 & 0.026 & 0.0701 & 0.0009 & 0.671 & 909 & 10 & 930 & 27 & 2 \\
\hline 57.1 & 320 & 192 & 0.60 & 179 & 000014 & 0.02 & 1.533 & 0.018 & 0.3107 & 0.0010 & 0.6522 & 0.0078 & 27.933 & 0.348 & 0.3106 & 0.0010 & 0.965 & 3237 & 31 & 3524 & 5 & 8 \\
\hline 58.1 & 367 & 314 & 0.86 & 27 & 0.000098 & 0.05 & 11.806 & 0.150 & 0.0583 & 0.0009 & 0.0847 & 0.0011 & & & & & & 524 & 7 & & & \\
\hline 59.1 & 150 & 187 & 1.24 & 15 & 0.000248 & 0.58 & 8.871 & 0.139 & 0.0670 & 0.0014 & 0.1121 & 0.0018 & & & & & & 685 & 10 & & & \\
\hline 60.1 & 286 & 468 & 1.63 & 116 & 0.000030 & 0.04 & 2.122 & 0.026 & 0.1646 & 0.0008 & 0.4710 & 0.0058 & 10.667 & 0.143 & 0.1643 & 0.0009 & 0.917 & 2488 & 25 & 2500 & 9 & 0 \\
\hline 61.1 & 193 & 188 & 0.98 & 54 & 00083 & 0.13 & 3.091 & 042 & 0.1226 & & 0.3231 & 0.0044 & 5.411 & 0.091 & 0.1215 & 0.0012 & 0.812 & 1805 & 22 & 1978 & 17 & 9 \\
\hline 62.1 & 101 & 154 & 1.52 & 8 & 0.000466 & 0.43 & 10.956 & 0.197 & 0.0623 & 0.0018 & 0.0909 & 0.0017 & & & & & & 561 & 10 & & & \\
\hline 63.1 & 261 & 234 & 0.90 & 74 & 0.000034 & 0.05 & 3.045 & 0.039 & 0.1155 & 0.0008 & 0.3282 & 0.0042 & 5.208 & 0.077 & 0.1151 & 0.0009 & 0.855 & 1830 & 20 & 1881 & 14 & 3 \\
\hline 64.1 & 196 & 115 & 0.59 & 81 & 0.000087 & 0.12 & 2.090 & 0.028 & 0.1665 & 0.0010 & 0.4779 & 0.0064 & 10.901 & 0.161 & 0.1654 & 0.0011 & 0.902 & 2518 & 28 & 2512 & 11 & 0 \\
\hline 65.1 & 187 & 98 & 0.53 & 26 & 0.000261 & 0.45 & 6.098 & 0.086 & 0.0706 & 0.0011 & 0.1638 & 0.0025 & 1.580 & 0.066 & 0.0699 & 0.0022 & 0.762 & 978 & 14 & 926 & 65 & -6 \\
\hline 66.1 & 827 & 17 & 0.02 & 69 & 0.000037 & 0.22 & 10.338 & 0.117 & 0.0615 & 0.0006 & 0.0965 & 0.0011 & & & & & & 594 & 7 & & & \\
\hline 67.1 & 142 & 82 & 0.57 & 59 & 0.000020 & 0.03 & 2.085 & 0.030 & 0.1674 & 0.0016 & 0.4794 & 0.0069 & 11.050 & 0.191 & 0.1672 & 0.0016 & 0.829 & 2525 & 30 & 2529 & 16 & 0 \\
\hline
\end{tabular}

Notes: $\quad$ 1. Uncertainties given at the one $\sigma$ level.

2. Error in $\mathrm{FC} 1$ reference zircon calibration was $0.40 \%$ for the analytical session. (not included in above errors but required when comparing ${ }^{206} \mathrm{~Pb} /{ }^{238} \mathrm{U}$ data from different mounts).

3. $\mathrm{f}_{206} \%$ denotes the percentage of ${ }^{206} \mathrm{~Pb}$ that is common $\mathrm{Pb}$.

4. For areas older than $\sim 800 \mathrm{Ma}$ correction for common $\mathrm{Pb}$ made using the measured ${ }^{204} \mathrm{~Pb} /{ }^{206} \mathrm{~Pb}$ ratio.

5. For areas younger than $\sim 800 \mathrm{Ma}$ correction for common $\mathrm{Pb}$ made using the measured ${ }^{238} \mathrm{U} /{ }^{206} \mathrm{~Pb}$ and ${ }^{207} \mathrm{~Pb} /{ }^{206} \mathrm{~Pb}$ ratios following Tera and Wasserburg (1972) as outlined in Williams (1998).

6. For $\%$ Disc, $0 \%$ denotes a concordant analysis. 
Table 6. SHRIMP U-Pb data for zircon grains from sample NY-11.

\begin{tabular}{|c|c|c|c|c|c|c|c|c|c|c|c|c|c|c|c|c|c|c|c|c|c|c|}
\hline \multirow[b]{2}{*}{$\begin{array}{c}\text { Grain. } \\
\text { spot }\end{array}$} & \multirow[b]{2}{*}{$\underset{(\mathrm{ppm})}{\cup}$} & \multirow[b]{2}{*}{$\begin{array}{c}\text { Th } \\
\text { (ppm) }\end{array}$} & \multirow[b]{2}{*}{$\mathrm{Th} / \mathrm{U}$} & \multirow[b]{2}{*}{$\begin{array}{l}\mathrm{Pb}^{\star} \\
(\mathrm{ppm})\end{array}$} & \multirow[b]{2}{*}{$\begin{array}{l}{ }^{204} \mathrm{~Pb} / \\
{ }^{206} \mathrm{~Pb}\end{array}$} & \multirow[b]{2}{*}{$\begin{array}{l}f_{206} \\
\%\end{array}$} & & Total R & & & & & adiogeni & atios & & & & & & ge (Ma) & & \\
\hline & & & & & & & $\begin{array}{l}{ }^{238} \mathrm{U} / \\
{ }^{206} \mathrm{~Pb}\end{array}$ & \pm & $\begin{array}{l}{ }^{207} \mathrm{~Pb} / \\
{ }^{206} \mathrm{~Pb}\end{array}$ & \pm & $\begin{array}{c}{ }^{206} \mathrm{~Pb} / \\
{ }^{238} \mathrm{U}\end{array}$ & \pm & $\begin{array}{c}{ }^{207} \mathrm{~Pb} / \\
{ }^{235} \mathrm{U}\end{array}$ & \pm & $\begin{array}{l}{ }^{207} \mathrm{~Pb} / \\
{ }^{206} \mathrm{~Pb}\end{array}$ & \pm & $\rho$ & $\begin{array}{l}{ }^{206} \mathrm{~Pb} / \\
{ }^{238} \mathrm{U}\end{array}$ & \pm & $\begin{array}{l}{ }^{207} \mathrm{~Pb} / \\
{ }^{206} \mathrm{~Pb}\end{array}$ & \pm & $\begin{array}{c}\% \\
\text { Disc }\end{array}$ \\
\hline 1.1 & 211 & 232 & 1.10 & 29.6 & 0.000483 & 0.83 & 6.121 & 0.079 & 0.0798 & 0.0010 & 0.1620 & 0.0021 & 1.628 & 0.059 & 0.0729 & 0.0025 & 0.361 & 968 & 12 & 1010 & 69 & 4 \\
\hline 2.1 & 1018 & 441 & 0.43 & 74.6 & 0.000140 & 0.25 & 11.726 & 0.131 & 0.0602 & 0.0005 & 0.0851 & 0.0010 & & & & & & 526 & 6 & & & \\
\hline 3.1 & 211 & 232 & 1.10 & 24.8 & 0.000540 & 0.94 & 7.301 & 0.094 & 0.0736 & 0.0010 & 0.1361 & 0.0018 & 1.290 & 0.028 & 0.0687 & 0.0012 & 0.588 & 823 & 12 & 890 & 37 & 8 \\
\hline 4.1 & 241 & 87 & 0.36 & 64.2 & 0.000313 & 0.49 & 3.229 & 0.040 & 0.1124 & 0.0009 & 0.3082 & 0.0038 & 4.596 & 0.084 & 0.1082 & 0.0014 & 0.679 & 1732 & 19 & 1769 & 24 & 2 \\
\hline 5.1 & 152 & 97 & 0.64 & 42.3 & 0.000173 & 0.27 & 3.097 & 0.044 & 0.1107 & 0.0011 & 0.3227 & 0.0046 & 4.911 & 0.084 & 0.1104 & 0.0011 & 0.828 & 1803 & 24 & 1805 & 17 & 0 \\
\hline 6.1 & 221 & 214 & 0.97 & 16.2 & 0.000100 & 0.18 & 11.742 & 0.160 & 0.0616 & 0.0012 & 0.0850 & 0.0012 & & & & & & 526 & 7 & & & \\
\hline 7.1 & 703 & 23 & 0.03 & 101.9 & 0.000078 & 0.13 & 5.927 & 0.067 & 0.0722 & 0.0005 & 0.1685 & 0.0019 & 1.651 & 0.025 & 0.0711 & 0.0007 & 0.762 & 1004 & 11 & 959 & 20 & -5 \\
\hline 8.1 & 933 & 490 & 0.53 & 118.2 & 0.000092 & 0.16 & 6.783 & 0.075 & 0.0716 & 0.0007 & 0.1472 & 0.0016 & 1.426 & 0.022 & 0.0702 & 0.0007 & 0.724 & 885 & 9 & 936 & 22 & 5 \\
\hline 9.1 & 323 & 71 & 0.22 & 84.5 & 0.000069 & 0.11 & 3.284 & 0.041 & 0.1182 & 0.0008 & 0.3042 & 0.0038 & 4.919 & 0.073 & 0.1173 & 0.0009 & 0.840 & 1712 & 19 & 1915 & 14 & 11 \\
\hline 10.1 & 1119 & 243 & 0.22 & 88.8 & 0.000085 & 0.15 & 10.827 & 0.118 & 0.0595 & 0.0005 & 0.0922 & 0.0010 & & & & & & 569 & 6 & & & \\
\hline 11.1 & 825 & 72 & 0.09 & 203.1 & 0.000075 & 0.12 & 3.491 & 0.038 & 0.1006 & 0.0005 & 0.2861 & 0.0031 & 3.927 & 0.050 & 0.0995 & 0.0007 & 0.856 & 1622 & 16 & 1616 & 12 & 0 \\
\hline 12.1 & 214 & 80 & 0.37 & 27.1 & 0.000420 & 0.73 & 6.765 & 0.094 & 0.0769 & 0.0011 & 0.1467 & 0.0021 & 1.435 & 0.055 & 0.0709 & 0.0025 & 0.372 & 883 & 12 & 956 & 72 & 8 \\
\hline 13.1 & 461 & 273 & 0.59 & 117.2 & 0.000298 & 0.47 & 3.379 & 0.068 & 0.1073 & 0.0006 & 0.2945 & 0.0060 & 4.190 & 0.104 & 0.1032 & 0.0015 & 0.816 & 1664 & 30 & 1682 & 26 & 1 \\
\hline 14.1 & 366 & 227 & 0.62 & 78.7 & 0.000212 & 0.34 & 3.999 & 0.049 & 0.0924 & 0.0008 & 0.2495 & 0.0030 & 3.126 & 0.047 & 0.0909 & 0.0008 & 0.807 & 1436 & 17 & 1444 & 17 & 1 \\
\hline 15.1 & 571 & 175 & 0.31 & 71.6 & 0.000301 & 0.52 & 6.848 & 0.077 & 0.0698 & 0.0006 & 0.1455 & 0.0016 & 1.337 & 0.021 & 0.0667 & 0.0007 & 0.713 & 875 & 10 & 827 & 23 & -6 \\
\hline 16.1 & 155 & 159 & 1.03 & 41.3 & 0.000496 & 0.78 & 3.221 & 0.043 & 0.1108 & 0.0011 & 0.3080 & 0.0041 & 4.418 & 0.104 & 0.1040 & 0.0020 & 0.569 & 1731 & 20 & 1697 & 36 & -2 \\
\hline 17.1 & 632 & 237 & 0.37 & 97.1 & 0.000601 & 1.02 & 5.589 & 0.064 & 0.0833 & 0.0012 & 0.1771 & 0.0021 & 1.826 & 0.059 & 0.0748 & 0.0022 & 0.361 & 1051 & 11 & 1062 & 61 & 1 \\
\hline 18.1 & 389 & 31 & 0.08 & 82.4 & 0.000997 & 1.63 & 4.053 & 0.047 & 0.1040 & 0.0018 & 0.2427 & 0.0029 & 3.015 & 0.121 & 0.0901 & 0.0034 & 0.297 & 1401 & 15 & 1427 & 73 & 2 \\
\hline 19.1 & 442 & 311 & 0.70 & 101.1 & 0.000918 & 1.48 & 3.755 & 0.043 & 0.1063 & 0.0022 & 0.2623 & 0.0032 & 3.382 & 0.175 & 0.0935 & 0.0047 & 0.237 & 1502 & 16 & 1498 & 95 & 0 \\
\hline 20.1 & 1159 & 5 & 0.00 & 300.9 & 0.000104 & 0.16 & 3.308 & 0.035 & 0.1053 & 0.0004 & 0.3018 & 0.0032 & 4.321 & 0.051 & 0.1039 & 0.0005 & 0.899 & 1700 & 16 & 1694 & 10 & 0 \\
\hline 21.1 & 429 & 149 & 0.35 & 71.4 & 0.000143 & 0.24 & 5.166 & 0.060 & 0.0792 & 0.0007 & 0.1931 & 0.0022 & 2.055 & 0.035 & 0.0772 & 0.0010 & 0.680 & 1138 & 12 & 1126 & 25 & -1 \\
\hline 22.1 & 628 & 834 & 1.33 & 85.4 & 0.000137 & 0.23 & 6.313 & 0.074 & 0.0732 & 0.0006 & 0.1580 & 0.0018 & 1.553 & 0.026 & 0.0713 & 0.0009 & 0.698 & 946 & 10 & 965 & 24 & 2 \\
\hline 23.1 & 339 & 148 & 0.44 & 37.5 & 0.000387 & 0.68 & 7.760 & 0.097 & 0.0691 & 0.0014 & 0.1280 & 0.0016 & 1.122 & 0.041 & 0.0636 & 0.0022 & 0.343 & 776 & 9 & 727 & 74 & -7 \\
\hline 24.1 & 344 & 181 & 0.53 & 48.7 & 0.000295 & 0.51 & 6.079 & 0.073 & 0.0758 & 0.0008 & 0.1637 & 0.0020 & 1.614 & 0.046 & 0.0715 & 0.0018 & 0.429 & 977 & 11 & 973 & 52 & 0 \\
\hline 25.1 & 144 & 120 & 0.84 & 24.6 & 0.000411 & 0.69 & 5.010 & 0.075 & 0.0830 & 0.0012 & 0.1982 & 0.0030 & 2.110 & 0.085 & 0.0772 & 0.0029 & 0.379 & 1166 & 16 & 1126 & 74 & -3 \\
\hline 26.1 & 275 & 132 & 0.48 & 69.7 & 0.000367 & 0.58 & 3.385 & 0.044 & 0.1074 & 0.0012 & 0.2937 & 0.0038 & 4.144 & 0.089 & 0.1023 & 0.0018 & 0.601 & 1660 & 19 & 1667 & 32 & 0 \\
\hline 27.1 & 153 & 75 & 0.49 & 26.4 & 0.000424 & 0.71 & 4.975 & 0.074 & 0.0830 & 0.0012 & 0.1996 & 0.0030 & 2.118 & 0.076 & 0.0770 & 0.0025 & 0.419 & 1173 & 16 & 1121 & 65 & -5 \\
\hline 28.1 & 602 & 69 & 0.12 & 103.9 & 0.000121 & 0.20 & 4.975 & 0.056 & 0.0826 & 0.0006 & 0.2006 & 0.0023 & 2.238 & 0.035 & 0.0809 & 0.0009 & 0.718 & 1179 & 12 & 1219 & 21 & 3 \\
\hline 29.1 & 482 & 310 & 0.64 & 118.9 & 0.000284 & 0.45 & 3.484 & 0.043 & 0.1027 & 0.0006 & 0.2857 & 0.0036 & 3.892 & 0.064 & 0.0988 & 0.0010 & 0.762 & 1620 & 18 & 1601 & 20 & -1 \\
\hline 30.1 & 148 & 187 & 1.26 & 12.0 & 0.003464 & 6.18 & 10.633 & 0.175 & 0.1070 & 0.0079 & 0.0882 & 0.0018 & & & & & & 545 & 11 & & & \\
\hline 31.1 & 368 & 212 & 0.58 & 85.4 & 0.000742 & 1.19 & 3.700 & 0.043 & 0.1035 & 0.0012 & 0.2670 & 0.0032 & 3.433 & 0.110 & 0.0932 & 0.0028 & 0.373 & 1526 & 16 & 1493 & 56 & -2 \\
\hline 32.1 & 757 & 193 & 0.25 & 62.3 & 0.001670 & 2.97 & 10.427 & 0.117 & 0.0826 & 0.0046 & 0.0933 & 0.0011 & & & & & & 575 & 7 & & & \\
\hline 33.1 & 399 & 344 & 0.86 & 38.9 & 0.001637 & 2.89 & 8.821 & 0.112 & 0.0850 & 0.0042 & 0.1101 & 0.0015 & & & & & & 673 & 9 & & & \\
\hline 34.1 & 501 & 76 & 0.15 & 49.5 & 0.000289 & 0.51 & 8.696 & 0.183 & 0.0628 & 0.0007 & 0.1144 & 0.0024 & & & & & & 698 & 14 & & & \\
\hline 35.1 & 607 & 272 & 0.45 & 149.5 & 0.000329 & 0.52 & 3.486 & 0.038 & 0.1116 & 0.0007 & 0.2854 & 0.0032 & 4.216 & 0.065 & 0.1072 & 0.0011 & 0.720 & 1618 & 16 & 1752 & 19 & 8 \\
\hline 36.1 & 76 & 23 & 0.30 & 12.1 & 0.002256 & 3.83 & 5.431 & 0.092 & 0.1006 & 0.0047 & 0.1772 & 0.0030 & 1.732 & 0.156 & 0.0709 & 0.0063 & 0.189 & 1052 & 19 & 954 & 181 & -10 \\
\hline 37.1 & 123 & 51 & 0.41 & 43.4 & 0.000431 & 0.64 & 2.444 & 0.034 & 0.1688 & 0.0015 & 0.4066 & 0.0058 & 9.159 & 0.170 & 0.1634 & 0.0020 & 0.763 & 2199 & 26 & 2491 & 20 & 12 \\
\hline 38.1 & 327 & 131 & 0.40 & 46.4 & 0.000942 & 1.61 & 6.051 & 0.074 & 0.0840 & 0.0019 & 0.1626 & 0.0021 & 1.580 & 0.087 & 0.0705 & 0.0038 & 0.229 & 971 & 11 & 942 & 110 & -3 \\
\hline 39.1 & 651 & 38 & 0.06 & 88.5 & 0.000340 & 0.58 & 6.324 & 0.073 & 0.0731 & 0.0006 & 0.1572 & 0.0018 & 1.479 & 0.033 & 0.0683 & 0.0013 & 0.522 & 941 & 10 & 876 & 39 & -7 \\
\hline 40.1 & 1525 & 175 & 0.12 & 211.6 & 0.000168 & 0.29 & 6.191 & 0.066 & 0.0741 & 0.0004 & 0.1611 & 0.0017 & 1.591 & 0.023 & 0.0717 & 0.0007 & 0.755 & 963 & 10 & 976 & 19 & 1 \\
\hline 41.1 & 285 & 307 & 1.08 & 48.5 & 0.000497 & 0.83 & 5.041 & 0.065 & 0.0854 & 0.0009 & 0.1967 & 0.0026 & 2.126 & 0.094 & 0.0784 & 0.0033 & 0.306 & 1158 & 14 & 1156 & 83 & 0 \\
\hline 42.1 & 642 & 82 & 0.13 & 186.6 & 0.000910 & 1.41 & 2.958 & 0.033 & 0.1252 & 0.0021 & 0.3333 & 0.0039 & 5.189 & 0.199 & 0.1129 & 0.0041 & 0.303 & 1854 & 19 & 1847 & 66 & 0 \\
\hline 43.1 & 617 & 170 & 0.28 & 133.0 & 0.000938 & 1.53 & 3.987 & 0.044 & 0.1027 & 0.0026 & 0.2470 & 0.0030 & 3.053 & 0.170 & 0.0897 & 0.0049 & 0.216 & 1423 & 15 & 1418 & 104 & 0 \\
\hline 44.1 & 963 & 125 & 0.13 & 252.5 & 0.000588 & 0.93 & 3.276 & 0.037 & 0.1103 & 0.0011 & 0.3031 & 0.0034 & 4.387 & 0.074 & 0.1050 & 0.0013 & 0.678 & 1707 & 18 & 1714 & 23 & 0 \\
\hline 45.1 & 157 & 257 & 1.63 & 24.5 & 0.002297 & 3.91 & 5.508 & 0.078 & 0.1080 & 0.0070 & 0.1745 & 0.0031 & 1.813 & 0.299 & 0.0754 & 0.0123 & 0.108 & 1037 & 17 & 1078 & 329 & 4 \\
\hline 46.1 & 172 & 125 & 0.73 & 28.1 & 0.002905 & 4.92 & 5.253 & 0.072 & 0.1195 & 0.0082 & 0.1810 & 0.0027 & 1.954 & 0.278 & 0.0783 & 0.0111 & 0.104 & 1072 & 15 & 1155 & 281 & 7 \\
\hline 47.1 & 528 & 362 & 0.69 & 77.2 & 0.000969 & 1.65 & 5.870 & 0.070 & 0.0844 & 0.0032 & 0.1675 & 0.0023 & 1.630 & 0.152 & 0.0706 & 0.0065 & 0.145 & 999 & 12 & 945 & 189 & -6 \\
\hline 48.1 & 349 & 262 & 0.75 & 53.8 & 0.000592 & 1.00 & 5.567 & 0.067 & 0.0825 & 0.0027 & 0.1778 & 0.0022 & 1.817 & 0.094 & 0.0741 & 0.0037 & 0.239 & 1055 & 12 & 1044 & 101 & -1 \\
\hline 49.1 & 317 & 128 & 0.40 & 53.2 & 0.000372 & 0.63 & 5.131 & 0.065 & 0.0851 & 0.0011 & 0.1937 & 0.0025 & 2.131 & 0.065 & 0.0798 & 0.0022 & 0.426 & 1141 & 14 & 1192 & 54 & 4 \\
\hline 50.1 & 84 & 10 & 0.12 & 16.6 & 0.007158 & 11.99 & 4.335 & 0.077 & 0.1783 & 0.0153 & 0.2037 & 0.0036 & 2.459 & 0.615 & 0.0876 & 0.0218 & 0.071 & 1195 & 36 & 1373 & 479 & 13 \\
\hline 51.1 & 562 & 153 & 0.27 & 86.0 & 0.000825 & 1.40 & 5.610 & 0.064 & 0.0852 & 0.0024 & 0.1757 & 0.0020 & 1.780 & 0.083 & 0.0735 & 0.0033 & 0.248 & 1044 & 11 & 1027 & 91 & -2 \\
\hline 52.1 & 396 & 210 & 0.53 & 31.0 & 0.003346 & 5.98 & 10.966 & 0.144 & 0.0988 & 0.0067 & 0.0866 & 0.0011 & & & & & & 536 & 9 & & & \\
\hline 53.1 & 103 & 5 & 0.05 & 9.1 & 0.002312 & 4.10 & 9.700 & 0.164 & 0.1321 & 0.0139 & 0.0935 & 0.0016 & & & & & & 576 & 14 & & & \\
\hline 54.1 & 121 & 86 & 0.71 & 49.3 & & 1.00 & 2.116 & 0.043 & 0.1734 & 0.0017 & 0.4680 & 0.0096 & 10.619 & 0.275 & 0.1646 & 0.0026 & 0.796 & 2475 & 42 & 2503 & 26 & 1 \\
\hline 55.1 & 107 & 51 & 0.47 & 16.5 & 002137 & 3.64 & 5.578 & 0.096 & 0.1004 & 0.0057 & 0.1731 & 0.0030 & 1.752 & 0.166 & 0.0734 & 0.0068 & 0.182 & 1029 & 21 & 1024 & 188 & 0 \\
\hline 56.1 & 615 & 682 & 1.11 & 237.5 & 0.000067 & 0.10 & 2.226 & 0.025 & 0.1725 & 0.0032 & 0.4488 & 0.0051 & 10.625 & 0.230 & 0.1717 & 0.0032 & 0.521 & 2390 & 23 & 2574 & 31 & 7 \\
\hline 57.1 & 575 & 332 & 0.58 & 80.8 & 0.000301 & 0.52 & 6.121 & 0.069 & 0.0758 & 0.0007 & 0.1625 & 0.0019 & 1.602 & 0.035 & 0.0715 & 0.0013 & 0.525 & 971 & 10 & 972 & 38 & 0 \\
\hline 58.1 & 109 & 67 & 0.62 & 13.0 & & 1.49 & 7.230 & 0.115 & 0.0757 & 0.0021 & 0.1363 & 0.0023 & 1.189 & 0.103 & 0.0633 & 0.0054 & 0.195 & 823 & 13 & 718 & 181 & -15 \\
\hline 59.1 & 427 & 197 & 0.46 & 69.9 & 0.000067 & 0.11 & 5.249 & 0.061 & 0.0770 & 0.0007 & 0.1903 & 0.0022 & 1.995 & 0.031 & 0.0761 & 0.0008 & 0.751 & 1123 & 12 & 1096 & 20 & -2 \\
\hline 60.1 & 410 & 175 & 0.43 & 56.0 & 0.000220 & 0.38 & 6.284 & 0.078 & 0.0719 & 0.0016 & 0.1585 & 0.0020 & 1.503 & 0.046 & 0.0688 & 0.0019 & 0.408 & 949 & 11 & 891 & 58 & -6 \\
\hline
\end{tabular}

Notes : $\quad$ 1. Uncertainties given at the one $\sigma$ level.

2. Error in TEMORA reference zircon calibration was $0.44 \%$ for the analytical session.

(not included in above errors but required when comparing $206 \mathrm{~Pb} / 238 \mathrm{U}$ data from different mounts).

3. f206 \% denotes the percentage of $206 \mathrm{~Pb}$ that is common $\mathrm{Pb}$.

4. Correction for common $\mathrm{Pb}$ made using the measured $204 \mathrm{~Pb} / 206 \mathrm{~Pb}$ ratio.

5. For $\%$ Disc, $0 \%$ denotes a concordant analysis. 
Table 7. SHRIMP U-Pb data for zircon grains from sample WL-270.

\begin{tabular}{|c|c|c|c|c|c|c|c|c|c|c|c|c|c|c|c|c|c|c|c|c|c|c|}
\hline \multirow[b]{2}{*}{$\begin{array}{c}\text { Grain. } \\
\text { spot }\end{array}$} & \multirow[b]{2}{*}{$\begin{array}{c}\mathrm{U} \\
(\mathrm{ppm})\end{array}$} & \multirow[b]{2}{*}{$\begin{array}{c}\text { Th } \\
(\mathrm{ppm})\end{array}$} & \multirow[b]{2}{*}{$\mathrm{Th} / \mathrm{U}$} & \multirow[b]{2}{*}{$\begin{array}{c}\mathrm{Pb}^{\star} \\
(\mathrm{ppm})\end{array}$} & \multirow[b]{2}{*}{$\begin{array}{l}{ }^{204} \mathrm{~Pb} / \\
{ }^{206} \mathrm{~Pb}\end{array}$} & \multirow[b]{2}{*}{$\begin{array}{c}f_{206} \\
\%\end{array}$} & & Total R & & & & & adiogenic & Ratios & & & & & & ge (Ma) & & \\
\hline & & & & & & & $\begin{array}{l}{ }^{238} \mathrm{U} / \\
{ }^{206} \mathrm{~Pb}\end{array}$ & \pm & $\begin{array}{l}{ }^{207} \mathrm{~Pb} / \\
{ }^{206} \mathrm{~Pb}\end{array}$ & \pm & $\begin{array}{l}{ }^{206} \mathrm{~Pb} / \\
{ }^{238} \mathrm{U}\end{array}$ & \pm & $\begin{array}{c}{ }^{207} \mathrm{~Pb} / \\
{ }^{235} \mathrm{U}\end{array}$ & \pm & $\begin{array}{l}{ }^{207} \mathrm{~Pb} / \\
{ }^{206} \mathrm{~Pb}\end{array}$ & \pm & $\rho$ & $\begin{array}{c}{ }^{206} \mathrm{~Pb} / \\
{ }^{238} \mathrm{U}\end{array}$ & \pm & $\begin{array}{l}{ }^{207} \mathrm{~Pb} / \\
{ }^{206} \mathrm{~Pb}\end{array}$ & \pm & $\begin{array}{c}\% \\
\text { Disc }\end{array}$ \\
\hline 1.1 & 609 & 2 & 0.00 & 226 & 0.000055 & 0.08 & 2.318 & 0.026 & 0.1541 & 0.0007 & 0.4311 & 0.0049 & 9.119 & 0.112 & 0.1534 & 0.0007 & 0.924 & 2310 & 22 & 2385 & 8 & 3 \\
\hline 2.1 & 1505 & 233 & 0.15 & 358 & 0.000023 & 0.04 & 3.616 & 0.039 & 0.1020 & 0.0004 & 0.2764 & 0.0029 & 3.876 & 0.045 & 0.1017 & 0.0004 & 0.924 & 1573 & 15 & 1655 & 8 & 5 \\
\hline 3.1 & 504 & 362 & 0.72 & 103 & 0.000036 & 0.06 & 4.212 & 0.049 & 0.0905 & 0.0007 & 0.2373 & 0.0028 & 2.946 & 0.043 & 0.0900 & 0.0008 & 0.807 & 1372 & 14 & 1426 & 16 & 4 \\
\hline 4.1 & 1106 & 5 & 0.00 & 255 & 0.000074 & 0.12 & 3.725 & 0.041 & 0.1122 & 0.0005 & 0.2682 & 0.0029 & 4.110 & 0.050 & 0.1112 & 0.0006 & 0.886 & 1531 & 15 & 1818 & 10 & 16 \\
\hline 5.1 & 532 & 400 & 0.75 & 37 & 0.000301 & 0.54 & 12.444 & 0.155 & 0.0601 & 0.0009 & 0.0802 & 0.0010 & & & & & & 498 & 7 & & & \\
\hline 6.1 & 767 & 754 & 0.98 & 156 & 0.000089 & 0.15 & 4.223 & 0.047 & 0.0914 & 0.0006 & 0.2364 & 0.0027 & 2.938 & 0.040 & 0.0901 & 0.0007 & 0.818 & 1368 & 14 & 1429 & 15 & 4 \\
\hline 7.1 & 993 & 67 & 0.07 & 127 & 0.000099 & 0.17 & 6.696 & 0.106 & 0.0714 & 0.0006 & 0.1491 & 0.0024 & 1.439 & 0.029 & 0.0700 & 0.0008 & 0.800 & 896 & 13 & 928 & 24 & 3 \\
\hline 8.1 & 365 & 432 & 1.19 & 62 & 0.000257 & 0.43 & 5.012 & 0.064 & 0.0881 & 0.0010 & 0.1987 & 0.0026 & 2.315 & 0.058 & 0.0845 & 0.0018 & 0.518 & 1168 & 14 & 1304 & 41 & 10 \\
\hline 9.1 & 351 & 373 & 1.06 & 24 & 0.000344 & 0.62 & 12.750 & 0.169 & 0.0574 & 0.0011 & 0.0784 & 0.0010 & & & & & & 487 & 8 & & & \\
\hline 10.1 & 95 & 113 & 1.20 & 6 & 0.001209 & 2.17 & 12.823 & 0.268 & 0.0794 & 0.0029 & 0.0763 & 0.0020 & & & & & & 474 & 12 & & & \\
\hline 11.1 & 307 & 239 & 0.78 & 27 & 0.000994 & 1.76 & 9.602 & 0.130 & 0.0691 & 0.0012 & 0.1036 & 0.0014 & & & & & & 635 & 10 & & & \\
\hline 12.1 & 392 & 150 & 0.38 & 59 & 0.000203 & 0.34 & 5.736 & 0.075 & 0.0780 & 0.0009 & 0.1737 & 0.0023 & 1.799 & 0.049 & 0.0751 & 0.0018 & 0.487 & 1033 & 13 & 1071 & 48 & 4 \\
\hline 13.1 & 111 & 125 & 1.12 & 21 & 0.000141 & 0.23 & 4.637 & 0.075 & 0.0843 & 0.0015 & 0.2152 & 0.0035 & 2.442 & 0.064 & 0.0823 & 0.0017 & 0.623 & 1256 & 19 & 1253 & 40 & 0 \\
\hline 14.1 & 342 & 255 & 0.74 & 36 & 0.000029 & 0.05 & 8.223 & 0.106 & 0.0646 & 0.0010 & 0.1215 & 0.0016 & 1.075 & 0.023 & 0.0642 & 0.0011 & 0.604 & 739 & 9 & 747 & 36 & 1 \\
\hline 15.1 & 1949 & 1569 & 0.81 & 157 & 0.000042 & 0.07 & 10.658 & 0.114 & 0.0604 & 0.0005 & 0.0938 & 0.0010 & & & & & & 578 & 6 & & & \\
\hline 16.1 & 166 & 148 & 0.89 & 26 & 0.000284 & 0.48 & 5.392 & 0.080 & 0.0755 & 0.0013 & 0.1885 & 0.0028 & 2.292 & 0.063 & 0.0882 & 0.0021 & 0.539 & 1113 & 18 & 1387 & 45 & 20 \\
\hline 17.1 & 1664 & 667 & 0.40 & 136 & 0.000064 & 0.11 & 10.535 & 0.114 & 0.0595 & 0.0005 & 0.0949 & 0.0010 & & & & & & 585 & 6 & & & \\
\hline 18.1 & 998 & 399 & 0.40 & 188 & 0.000031 & 0.05 & 4.569 & 0.055 & 0.0826 & 0.0005 & 0.2187 & 0.0026 & 2.478 & 0.034 & 0.0822 & 0.0005 & 0.888 & 1275 & 14 & 1250 & 12 & -2 \\
\hline 19.1 & 1945 & 967 & 0.50 & 137 & 0.000082 & 0.15 & 12.196 & 0.131 & 0.0576 & 0.0005 & 0.0819 & 0.0009 & & & & & & 507 & 5 & & & \\
\hline 20.1 & 364 & 183 & 0.50 & 70 & 0.000009 & 0.01 & 4.438 & 0.057 & 0.0889 & 0.0008 & 0.2253 & 0.0029 & 2.759 & 0.047 & 0.0888 & 0.0010 & 0.754 & 1310 & 15 & 1400 & 22 & 6 \\
\hline 21.1 & 232 & 141 & 0.61 & 48 & 0.000125 & 0.20 & 4.111 & 0.054 & 0.0953 & 0.0010 & 0.2428 & 0.0032 & 3.133 & 0.060 & 0.0936 & 0.0013 & 0.691 & 1401 & 17 & 1500 & 26 & 7 \\
\hline 22.1 & 783 & 411 & 0.52 & 146 & 0.000062 & 0.10 & 4.616 & 0.052 & 0.0872 & 0.0009 & 0.2164 & 0.0024 & 2.577 & 0.040 & 0.0864 & 0.0009 & 0.729 & 1263 & 13 & 1346 & 20 & 6 \\
\hline 23.1 & 128 & 137 & 1.07 & 23 & 0.000379 & 0.63 & 4.745 & 0.075 & 0.0846 & 0.0015 & 0.2093 & 0.0033 & 2.291 & 0.060 & 0.0794 & 0.0016 & 0.606 & 1225 & 21 & 1182 & 41 & -4 \\
\hline 24.1 & 220 & 160 & 0.73 & 18 & 0.000254 & 0.45 & 10.754 & 0.157 & 0.0608 & 0.0013 & 0.0926 & 0.0014 & & & & & & 571 & 8 & & & \\
\hline 25.1 & 70 & 121 & 1.73 & 12 & 0.000122 & 0.21 & 5.161 & 0.100 & 0.0785 & 0.0020 & 0.1933 & 0.0038 & 2.047 & 0.084 & 0.0768 & 0.0028 & 0.476 & 1139 & 20 & 1115 & 72 & -2 \\
\hline 26.1 & 190 & 295 & 1.56 & 29 & 0.000133 & 0.23 & 5.654 & 0.081 & 0.0761 & 0.0013 & 0.1765 & 0.0025 & 1.806 & 0.054 & 0.0742 & 0.0020 & 0.479 & 1048 & 14 & 1047 & 53 & 0 \\
\hline 27.1 & 1267 & 317 & 0.25 & 180 & 0.000004 & 0.01 & 6.041 & 0.065 & 0.0745 & 0.0005 & 0.1655 & 0.0018 & 1.699 & 0.021 & 0.0744 & 0.0005 & 0.863 & 987 & 10 & 1054 & 13 & 6 \\
\hline 28.1 & 169 & 220 & 1.30 & 19 & 0.000036 & 0.06 & 7.553 & 0.116 & 0.0695 & 0.0014 & 0.1323 & 0.0020 & 1.258 & 0.046 & 0.0690 & 0.0023 & 0.419 & 801 & 12 & 898 & 69 & 11 \\
\hline 29.1 & 57 & 99 & 1.75 & 12 & 0.000899 & 1.47 & 4.118 & 0.190 & 0.0755 & 0.0020 & 0.2567 & 0.0118 & 4.176 & 0.279 & 0.1180 & 0.0057 & 0.690 & 1473 & 76 & 1926 & 87 & 24 \\
\hline 30.1 & 63 & 22 & 0.35 & 12 & 0.003444 & 5.76 & 4.613 & 0.094 & 0.1287 & 0.0061 & 0.2043 & 0.0048 & 2.255 & 0.381 & 0.0801 & 0.0134 & 0.140 & 1198 & 26 & 1198 & 330 & 0 \\
\hline 31.1 & 2006 & 303 & 0.15 & 551 & 0.000017 & 0.03 & 3.125 & 0.034 & 0.1165 & 0.0004 & 0.3199 & 0.0035 & 5.129 & 0.059 & 0.1163 & 0.0004 & 0.948 & 1789 & 17 & 1900 & 6 & 6 \\
\hline 32.1 & 243 & 40 & 0.16 & 27 & 0.000196 & 0.34 & 7.762 & 0.124 & 0.0672 & 0.0013 & 0.1287 & 0.0021 & 1.179 & 0.030 & 0.0664 & 0.0013 & 0.630 & 781 & 12 & 820 & 41 & 5 \\
\hline 33.1 & 445 & 387 & 0.87 & 67 & 0.000136 & 0.23 & 5.693 & 0.073 & 0.0769 & 0.0009 & 0.1752 & 0.0022 & 1.811 & 0.034 & 0.0749 & 0.0010 & 0.683 & 1041 & 12 & 1067 & 27 & 2 \\
\hline 34.1 & 1529 & 332 & 0.22 & 238 & 0.000055 & 0.09 & 5.518 & 0.060 & 0.0757 & 0.0005 & 0.1810 & 0.0020 & 1.870 & 0.024 & 0.0749 & 0.0005 & 0.838 & 1073 & 11 & 1066 & 14 & -1 \\
\hline 35.1 & 546 & 645 & 1.18 & 59 & 0.000080 & 0.14 & 8.008 & 0.098 & 0.0633 & 0.0008 & 0.1247 & 0.0015 & & & & & & 758 & 9 & & & \\
\hline 36.1 & 970 & 1626 & 1.68 & 183 & 0.000004 & 0.01 & 4.546 & 0.049 & 0.0836 & 0.0004 & 0.2200 & 0.0024 & 2.533 & 0.031 & 0.0835 & 0.0005 & 0.891 & 1282 & 13 & 1282 & 11 & 0 \\
\hline 37.1 & 342 & 233 & 0.68 & 54 & 0.000095 & 0.16 & 5.458 & 0.071 & 0.0786 & 0.0010 & 0.1829 & 0.0024 & 1.948 & 0.041 & 0.0772 & 0.0013 & 0.619 & 1083 & 13 & 1127 & 33 & 4 \\
\hline 38.1 & 431 & 322 & 0.75 & 71 & 0.000076 & 0.13 & 5.224 & 0.069 & 0.0802 & 0.0010 & 0.1912 & 0.0025 & 2.086 & 0.039 & 0.0791 & 0.0011 & 0.705 & 1128 & 14 & 1176 & 26 & 4 \\
\hline 39.1 & 326 & 206 & 0.63 & 23 & 0.000483 & 0.87 & 12.413 & 0.172 & 0.0594 & 0.0012 & 0.0808 & 0.0011 & & & & & & 501 & 7 & & & \\
\hline 40.1 & 411 & 508 & 1.24 & 29 & 0.000118 & 0.21 & 12.195 & 0.164 & 0.0607 & 0.0012 & 0.0818 & 0.0011 & & & & & & 507 & 7 & & & \\
\hline 41.1 & 203 & 35 & 0.17 & 113 & 0.000085 & 0.11 & 1.547 & 0.021 & 0.2643 & 0.0015 & 0.6459 & 0.0088 & 23.455 & 0.348 & 0.2634 & 0.0015 & 0.920 & 3212 & 35 & 3267 & 9 & 2 \\
\hline 42.1 & 446 & 129 & 0.29 & 36 & 0.000324 & 0.58 & 10.667 & 0.145 & 0.0585 & 0.0010 & 0.0938 & 0.0013 & & & & & & 578 & 8 & & & \\
\hline 43.1 & 670 & 203 & 0.30 & 154 & 0.000078 & 0.13 & 3.740 & 0.044 & 0.1133 & 0.0007 & 0.2671 & 0.0032 & 4.134 & 0.057 & 0.1123 & 0.0008 & 0.852 & 1526 & 16 & 1837 & 13 & 17 \\
\hline 44.1 & 1107 & 1123 & 1.01 & 242 & 0.000054 & 0.09 & 3.931 & 0.044 & 0.0959 & 0.0005 & 0.2541 & 0.0028 & 3.335 & 0.042 & 0.0952 & 0.0005 & 0.891 & 1460 & 15 & 1532 & 11 & 5 \\
\hline 45.1 & 129 & 73 & 0.57 & 13 & 0.000494 & 0.87 & 8.489 & 0.146 & 0.0696 & 0.0022 & 0.1177 & 0.0020 & & & & & & 717 & 13 & & & \\
\hline 46.1 & 105 & 114 & 1.09 & 10 & 0.000524 & 0.93 & 9.098 & 0.214 & 0.0711 & 0.0019 & 0.1089 & 0.0026 & & & & & & 666 & 15 & & & \\
\hline 47.1 & 541 & 212 & 0.39 & 57 & 0.000052 & 0.09 & 8.155 & 0.093 & 0.0658 & 0.0007 & 0.1225 & 0.0014 & & & & & & 745 & 8 & & & \\
\hline 48.1 & 1870 & 327 & 0.17 & 332 & 0.000035 & 0.06 & 4.835 & 0.051 & 0.0764 & 0.0003 & 0.2067 & 0.0022 & 2.164 & 0.025 & 0.0759 & 0.0004 & 0.898 & 1211 & 12 & 1093 & 10 & -11 \\
\hline 49.1 & 131 & 46 & 0.35 & 36 & 0.000194 & 0.30 & 3.143 & 0.054 & 0.1432 & 0.0018 & 0.3172 & 0.0055 & 6.151 & 0.168 & 0.1406 & 0.0030 & 0.632 & 1776 & 27 & 2235 & 37 & 21 \\
\hline 50.1 & 341 & 631 & 1.85 & 53 & 0.000045 & 0.08 & 5.493 & 0.071 & 0.0770 & 0.0010 & 0.1819 & 0.0024 & 1.915 & 0.048 & 0.0764 & 0.0016 & 0.514 & 1077 & 13 & 1104 & 43 & 2 \\
\hline 51.1 & 2230 & 92 & 0.04 & 727 & 0.000015 & 0.02 & 2.636 & 0.028 & 0.1628 & 0.0004 & 0.3793 & 0.0040 & 8.507 & 0.093 & 0.1626 & 0.0005 & 0.967 & 2073 & 19 & 2483 & 5 & 17 \\
\hline 52.1 & 1253 & 228 & 0.18 & 162 & 0.000051 & 0.09 & 6.660 & 0.076 & 0.0730 & 0.0006 & 0.1500 & 0.0017 & 1.495 & 0.021 & 0.0723 & 0.0006 & 0.818 & 901 & 10 & 993 & 16 & 9 \\
\hline 53.1 & 472 & 319 & 0.68 & 88 & 0.000176 & 0.29 & 4.599 & 0.056 & 0.0846 & 0.0008 & 0.2168 & 0.0026 & 2.455 & 0.046 & 0.0821 & 0.0011 & 0.657 & 1265 & 14 & 1249 & 27 & -1 \\
\hline 54.1 & 405 & 297 & 0.73 & 200 & 0.000077 & 0.10 & 1.739 & 0.022 & 0.2413 & 0.0012 & 0.5745 & 0.0071 & 19.050 & 0.256 & 0.2405 & 0.0012 & 0.926 & 2926 & 29 & 3123 & 8 & 6 \\
\hline 55.1 & 504 & 633 & 1.26 & 34 & - & $<0.01$ & 12.899 & 0.172 & 0.0612 & 0.0011 & 0.0776 & 0.0010 & & & & & & 482 & 6 & & & \\
\hline 56.1 & 1486 & 297 & 0.20 & 242 & - & $<0.01$ & 5.284 & 0.058 & 0.0795 & 0.0005 & 0.1892 & 0.0021 & 2.074 & 0.026 & 0.0795 & 0.0005 & 0.863 & 1117 & 11 & 1184 & 13 & 6 \\
\hline 57.1 & 642 & 10 & 0.02 & 48 & - & $<0.01$ & 11.375 & 0.158 & 0.0626 & 0.0013 & 0.0879 & 0.0012 & & & & & & 543 & 7 & & & \\
\hline 58.1 & 321 & 148 & 0.46 & 50 & 0.000531 & 0.90 & 5.556 & 0.093 & 0.0939 & 0.0016 & 0.1784 & 0.0031 & 2.126 & 0.088 & 0.0864 & 0.0033 & 0.413 & 1058 & 17 & 1348 & 73 & 22 \\
\hline 59.1 & 599 & 80 & 0.13 & 83 & 0.000080 & 0.14 & 6.170 & 0.080 & 0.0772 & 0.0010 & 0.1618 & 0.0021 & 1.698 & 0.038 & 0.0761 & 0.0014 & 0.585 & 967 & 12 & 1097 & 36 & 12 \\
\hline 60.1 & 416 & 253 & 0.61 & 91 & 0.000073 & 0.12 & 3.943 & 0.051 & 0.0988 & 0.0018 & 0.2533 & 0.0033 & 3.415 & 0.076 & 0.0978 & 0.0018 & 0.585 & 1455 & 17 & 1582 & 34 & 8 \\
\hline
\end{tabular}

Notes : $\quad 1$. Uncertainties given at the one $\sigma$ level.

2. Error in TEMORA reference zircon calibration was $0.44 \%$ for the analytical session.

( not included in above errors but required when comparing ${ }^{206} \mathrm{~Pb} /{ }^{238} \mathrm{U}$ data from different mounts).

3. $\mathrm{f}_{206} \%$ denotes the percentage of ${ }^{206} \mathrm{~Pb}$ that is common $\mathrm{Pb}$.

4. Correction for common $\mathrm{Pb}$ made using the measured ${ }^{204} \mathrm{~Pb} / 206 \mathrm{~Pb}$ ratio

5. For $\%$ Disc, $0 \%$ denotes a concordant analysis. 
Table 8. SHRIMP U-Pb data for zircon grains from sample KU-2.

\begin{tabular}{|c|c|c|c|c|c|c|c|c|c|c|c|c|c|c|c|c|c|c|c|c|c|c|}
\hline \multirow[b]{2}{*}{$\begin{array}{c}\text { Grain. } \\
\text { spot }\end{array}$} & \multirow[b]{2}{*}{$\underset{(\mathrm{ppm})}{\mathrm{U}}$} & \multirow[b]{2}{*}{$\begin{array}{c}\text { Th } \\
(\mathrm{ppm})\end{array}$} & \multirow[b]{2}{*}{$T h / U$} & \multirow[b]{2}{*}{$\begin{array}{c}\mathrm{Pb}^{*} \\
(\mathrm{ppm})\end{array}$} & \multirow[b]{2}{*}{$\begin{array}{l}{ }^{204} \mathrm{~Pb} / \\
{ }^{206} \mathrm{~Pb}\end{array}$} & \multirow[b]{2}{*}{$\begin{array}{l}\mathrm{f}_{206} \\
\%\end{array}$} & \multicolumn{4}{|c|}{ Total Ratios } & \multicolumn{6}{|c|}{ Radiogenic Ratios } & \multicolumn{5}{|c|}{ Age (Ma) } & \multirow[b]{2}{*}{$\begin{array}{c}\% \\
\text { Disc }\end{array}$} \\
\hline & & & & & & & $\begin{array}{l}{ }^{238} \mathrm{U} / \\
{ }^{206} \mathrm{~Pb}\end{array}$ & \pm & $\begin{array}{l}{ }^{207} \mathrm{~Pb} / \\
{ }^{206} \mathrm{~Pb}\end{array}$ & \pm & $\begin{array}{l}{ }^{206} \mathrm{~Pb} / \\
{ }^{238} \mathrm{U}\end{array}$ & \pm & $\begin{array}{c}{ }^{207} \mathrm{~Pb} / \\
{ }^{235} \mathrm{U}\end{array}$ & \pm & $\begin{array}{l}{ }^{207} \mathrm{~Pb} / \\
{ }^{206} \mathrm{~Pb}\end{array}$ & \pm & $\rho$ & $\begin{array}{l}{ }^{206} \mathrm{~Pb} / \\
{ }^{238} \mathrm{U}\end{array}$ & \pm & $\begin{array}{l}{ }^{207} \mathrm{~Pb} / \\
{ }^{206} \mathrm{~Pb}\end{array}$ & \pm & \\
\hline 1.1 & 82 & 93 & 1.13 & 24 & 0.001620 & 2.57 & 3.320 & 0.054 & 0.1238 & 0.0043 & 0.2934 & 0.0051 & 4.110 & 0.304 & 0.1016 & 0.0073 & 0.235 & 1659 & 25 & 1653 & 133 & 0 \\
\hline 2.1 & 359 & 223 & 0.62 & 32 & 0.000886 & 1.54 & 7.189 & 0.088 & 0.0789 & 0.0026 & 0.1370 & 0.0017 & 1.249 & 0.081 & 0.0662 & 0.0042 & 0.195 & 827 & 10 & 812 & 133 & -2 \\
\hline 3.1 & 302 & 519 & 1.72 & 2 & 0.000784 & 1.39 & 10.560 & 0.143 & 0.0689 & 0.0019 & 0.0934 & 0.0013 & & & & & & 575 & 8 & & & \\
\hline 4.1 & 347 & 95 & 0.27 & 91 & 0.000427 & 0.68 & 3.393 & 0.042 & 0.1073 & 0.0008 & 0.2927 & 0.0036 & 4.096 & 0.085 & 0.1015 & 0.0017 & 0.601 & 1655 & 18 & 1652 & 31 & 0 \\
\hline 5.1 & 425 & 332 & 0.78 & 78 & 0.000318 & 0.54 & 5.293 & 0.062 & 0.0820 & 0.0010 & 0.1879 & 0.0022 & 2.008 & 0.046 & 0.0775 & 0.0015 & 0.515 & 1110 & 12 & 1134 & 39 & 2 \\
\hline 6.1 & 364 & 67 & 0.18 & 103 & 0.000483 & 0.80 & 4.706 & 0.059 & 0.0839 & 0.0008 & 0.2112 & 0.0026 & 2.298 & 0.041 & 0.0789 & 0.0010 & 0.697 & 1235 & 15 & 1171 & 25 & -6 \\
\hline 7.1 & 306 & 273 & 0.89 & 253 & 0.001330 & 2.30 & 6.785 & 0.085 & 0.0883 & 0.0029 & 0.1440 & 0.0019 & 1.376 & 0.109 & 0.0693 & 0.0054 & 0.169 & 867 & 11 & 907 & 161 & 4 \\
\hline 8.1 & 644 & 313 & 0.49 & 3 & 0.001317 & 2.07 & 3.204 & 0.035 & 0.1302 & 0.0038 & 0.3056 & 0.0037 & 4.734 & 0.259 & 0.1123 & 0.0060 & 0.219 & 1719 & 18 & 1838 & 97 & 6 \\
\hline 9.1 & 827 & 206 & 0.25 & 3 & 0.000255 & 0.43 & 5.848 & 0.064 & 0.0816 & 0.0013 & 0.1703 & 0.0019 & 1.831 & 0.048 & 0.0780 & 0.0018 & 0.428 & 1014 & 10 & 1146 & 47 & 12 \\
\hline 10.1 & 664 & 217 & 0.33 & 3 & 0.000186 & 0.27 & 2.311 & 0.026 & 0.2462 & 0.0008 & 0.4316 & 0.0048 & 14.524 & 0.172 & 0.2441 & 0.0009 & 0.948 & 2313 & 22 & 3147 & 6 & 26 \\
\hline 11.1 & 545 & 221 & 0.41 & 6 & 0.000360 & 0.59 & 4.154 & 0.047 & 0.0955 & 0.0006 & 0.2393 & 0.0027 & 2.984 & 0.061 & 0.0904 & 0.0015 & 0.554 & 1383 & 14 & 1435 & 33 & 4 \\
\hline 12.1 & 65 & 38 & 0.58 & 188 & 0.006225 & 10.7 & 5.720 & 0.103 & 0.1439 & 0.0098 & 0.1615 & 0.0029 & 1.876 & 0.301 & 0.0843 & 0.0135 & 0.112 & 965 & 21 & 1299 & 310 & 26 \\
\hline 13.1 & 228 & 158 & 0.69 & 0 & 0.000545 & 0.97 & 9.762 & 0.131 & 0.0667 & 0.0012 & 0.1015 & 0.0014 & & & & & & 623 & 8 & & & \\
\hline 14.1 & 518 & 139 & 0.27 & 1 & 0.000115 & 0.16 & 1.928 & 0.022 & 0.1927 & 0.0007 & 0.5179 & 0.0059 & 13.660 & 0.165 & 0.1913 & 0.0008 & 0.945 & 2690 & 25 & 2753 & 6 & 2 \\
\hline 15.1 & 279 & 83 & 0.30 & 3 & 0.000157 & 0.24 & 2.843 & 0.034 & 0.1197 & 0.0010 & 0.3509 & 0.0042 & 5.687 & 0.090 & 0.1176 & 0.0012 & 0.761 & 1939 & 20 & 1919 & 18 & -1 \\
\hline 16.1 & 179 & 11 & 0.06 & 253 & 0.001218 & 2.17 & 10.963 & 0.162 & 0.0770 & 0.0016 & 0.0892 & 0.0015 & & & & & 0.137 & 551 & 9 & & & \\
\hline 17.1 & 812 & 113 & 0.14 & 3 & 0.000272 & 0.46 & 5.871 & 0.066 & 0.0832 & 0.0005 & 0.1695 & 0.0019 & 1.855 & 0.035 & 0.0793 & 0.0012 & 0.605 & 1010 & 11 & 1181 & 29 & 14 \\
\hline 18.1 & 82 & 44 & 0.54 & 4 & 0.002552 & 4.34 & 5.514 & 0.105 & 0.1087 & 0.0044 & 0.1734 & 0.0033 & 1.767 & 0.159 & 0.0739 & 0.0065 & 0.212 & 1031 & 22 & 1039 & 177 & 1 \\
\hline 19.1 & 439 & 150 & 0.34 & 3 & 0.000384 & 0.66 & 6.185 & 0.074 & 0.0775 & 0.0009 & 0.1606 & 0.0019 & 1.594 & 0.044 & 0.0720 & 0.0018 & 0.435 & 960 & 11 & 986 & 51 & 3 \\
\hline 20.1 & 295 & 178 & 0.60 & 5 & 0.000252 & 0.44 & 7.912 & 0.100 & 0.0690 & 0.0010 & 0.1258 & 0.0016 & & & & & & 764 & 9 & & & \\
\hline 21.1 & 848 & 141 & 0.17 & 73 & 0.000224 & 0.40 & 9.713 & 0.108 & 0.0624 & 0.0006 & 0.1027 & 0.0011 & & & & & & 630 & 7 & & & \\
\hline 22.1 & 133 & 159 & 1.20 & 2 & 0.000036 & 0.06 & 7.735 & 0.120 & 0.0721 & 0.0016 & 0.1292 & 0.0020 & & & & & & 783 & 12 & & & \\
\hline 23.1 & 266 & 46 & 0.17 & 68 & 0.000692 & 1.22 & 8.524 & 0.112 & 0.0764 & 0.0012 & 0.1159 & 0.0016 & & & & & & 707 & 9 & & & \\
\hline 24.1 & 548 & 198 & 0.36 & 6 & 0.000105 & 0.15 & 2.024 & 0.023 & 0.2577 & 0.0009 & 0.4933 & 0.0057 & 17.445 & 0.213 & 0.2565 & 0.0010 & 0.951 & 2585 & 25 & 3225 & 6 & 20 \\
\hline 25.1 & 556 & 228 & 0.41 & 42 & 0.000091 & 0.15 & 4.275 & 0.050 & 0.1370 & 0.0008 & 0.2336 & 0.0027 & 4.375 & 0.059 & 0.1358 & 0.0009 & 0.866 & 1353 & 14 & 2175 & 12 & 38 \\
\hline 26.1 & 464 & 284 & 0.61 & 5 & 0.000090 & 0.14 & 3.636 & 0.042 & 0.1064 & 0.0007 & 0.2746 & 0.0032 & 3.983 & 0.057 & 0.1052 & 0.0009 & 0.808 & 1564 & 16 & 1718 & 15 & 9 \\
\hline 27.1 & 611 & 794 & 1.30 & 3 & 0.001056 & 1.91 & 14.444 & 0.174 & 0.0750 & 0.0010 & 0.0679 & 0.0009 & & & & & & 424 & 5 & & & \\
\hline 28.1 & 467 & 310 & 0.66 & 3 & 0.000455 & 0.78 & 6.304 & 0.075 & 0.0814 & 0.0008 & 0.1574 & 0.0019 & 1.625 & 0.045 & 0.0749 & 0.0019 & 0.430 & 942 & 10 & 1065 & 51 & 12 \\
\hline 29.1 & 920 & 811 & 0.88 & 4 & 0.000044 & 0.07 & 5.420 & 0.059 & 0.0824 & 0.0005 & 0.1844 & 0.0020 & 2.079 & 0.027 & 0.0818 & 0.0006 & 0.837 & 1091 & 11 & 1240 & 14 & 12 \\
\hline 30.1 & 658 & 29 & 0.04 & 4 & 0.000226 & 0.40 & 9.278 & 0.108 & 0.0669 & 0.0008 & 0.1074 & 0.0013 & & & & & & 657 & 7 & & & \\
\hline 31.1 & 1824 & 92 & 0.05 & 4 & 0.000047 & 0.07 & 3.079 & 0.032 & 0.1135 & 0.0004 & 0.3245 & 0.0034 & 5.049 & 0.056 & 0.1128 & 0.0004 & 0.951 & 1812 & 17 & 1845 & 6 & 2 \\
\hline 32.1 & 1457 & 43 & 0.03 & 8 & 0.000573 & 0.96 & 5.134 & 0.055 & 0.0850 & 0.0011 & 0.1932 & 0.0021 & 2.091 & 0.043 & 0.0785 & 0.0014 & 0.517 & 1139 & 11 & 1160 & 35 & 2 \\
\hline 33.1 & 325 & 533 & 1.64 & 4 & 0.000664 & 1.19 & 12.666 & 0.165 & 0.0726 & 0.0034 & 0.0780 & 0.0011 & & & & & & 484 & 6 & & & \\
\hline 34.1 & 671 & 455 & 0.68 & 138 & 0.000299 & 0.48 & 3.752 & 0.042 & 0.1032 & 0.0009 & 0.2652 & 0.0030 & 3.623 & 0.061 & 0.0991 & 0.0012 & 0.670 & 1517 & 15 & 1607 & 23 & 6 \\
\hline 35.1 & 767 & 280 & 0.37 & 91 & 0.000076 & 0.13 & 5.899 & 0.066 & 0.0749 & 0.0006 & 0.1693 & 0.0019 & 1.723 & 0.025 & 0.0738 & 0.0007 & 0.754 & 1008 & 10 & 1037 & 20 & 3 \\
\hline 36.1 & 317 & 246 & 0.78 & 50 & 0.000404 & 0.71 & 9.182 & 0.127 & 0.0664 & 0.0011 & 0.1081 & 0.0015 & & & & & & 662 & 9 & & & \\
\hline 37.1 & 1051 & 303 & 0.29 & 52 & 0.000105 & 0.18 & 7.547 & 0.090 & 0.0821 & 0.0009 & 0.1323 & 0.0016 & 1.470 & 0.028 & 0.0806 & 0.0012 & 0.628 & 801 & 9 & 1212 & 29 & 34 \\
\hline 38.1 & 209 & 104 & 0.50 & 5 & 0.000382 & 0.59 & 3.028 & 0.039 & 0.1111 & 0.0012 & 0.3295 & 0.0042 & 4.968 & 0.085 & 0.1093 & 0.0012 & 0.758 & 1836 & 22 & 1788 & 20 & -3 \\
\hline 39.1 & 808 & 692 & 0.86 & 21 & 0.000056 & 0.10 & 11.603 & 0.132 & 0.0602 & 0.0007 & 0.0861 & 0.0010 & & & & & & 532 & 6 & & & \\
\hline 40.1 & 496 & 88 & 0.18 & 2 & 0.000079 & 0.13 & 3.398 & 0.040 & 0.1072 & 0.0007 & 0.2939 & 0.0035 & 4.301 & 0.060 & 0.1061 & 0.0008 & 0.852 & 1661 & 17 & 1734 & 13 & 4 \\
\hline 41.1 & 446 & 55 & 0.12 & 11 & 0.000049 & 0.08 & 3.257 & 0.042 & 0.1175 & 0.0096 & 0.3068 & 0.0040 & 4.942 & 0.411 & 0.1168 & 0.0096 & 0.156 & 1725 & 20 & 1908 & 148 & 10 \\
\hline 42.1 & 648 & 160 & 0.25 & 3 & 0.000002 & $<0.01$ & 3.992 & 0.045 & 0.0960 & 0.0008 & 0.2505 & 0.0028 & 3.315 & 0.045 & 0.0960 & 0.0008 & 0.816 & 1441 & 14 & 1548 & 15 & 7 \\
\hline 43.1 & 654 & 254 & 0.39 & 46 & 0.000106 & 0.18 & 5.592 & 0.063 & 0.0798 & 0.0006 & 0.1785 & 0.0020 & 1.928 & 0.035 & 0.0783 & 0.0011 & 0.617 & 1059 & 11 & 1155 & 29 & 8 \\
\hline 44.1 & 678 & 622 & 0.92 & 54 & 0.000158 & 0.27 & 5.067 & 0.057 & 0.0894 & 0.0006 & 0.1968 & 0.0022 & 2.366 & 0.041 & 0.0872 & 0.0012 & 0.644 & 1158 & 12 & 1365 & 26 & 15 \\
\hline 45.1 & 1913 & 1381 & 0.72 & 3 & 0.000465 & 0.81 & 7.123 & 0.075 & 0.1063 & 0.0005 & 0.1393 & 0.0015 & 1.917 & 0.032 & 0.0999 & 0.0013 & 0.646 & 840 & 8 & 1621 & 23 & 48 \\
\hline 46.1 & 99 & 111 & 1.12 & 16 & 0.005400 & 9.45 & 7.368 & 0.136 & 0.1465 & 0.0519 & 0.1229 & 0.0050 & & & & & & 747 & 29 & & & \\
\hline 47.1 & 532 & 60 & 0.11 & 22 & 0.000239 & 0.41 & 6.892 & 0.084 & 0.0778 & 0.0014 & 0.1445 & 0.0018 & 1.482 & 0.041 & 0.0744 & 0.0018 & 0.445 & 870 & 10 & 1052 & 49 & 17 \\
\hline 48.1 & 590 & 150 & 0.25 & 40 & 0.000213 & 0.37 & 7.851 & 0.091 & 0.0717 & 0.0007 & 0.1269 & 0.0015 & & & & & & 770 & 8 & & & \\
\hline 49.1 & 398 & 260 & 0.65 & 29 & 0.000392 & 0.70 & 9.864 & 0.122 & 0.0678 & 0.0009 & 0.1007 & 0.0013 & & & & & & 618 & 7 & & & \\
\hline 50.1 & 570 & 478 & 0.84 & 10 & 0.000204 & 0.35 & 5.707 & 0.065 & 0.0770 & 0.0007 & 0.1746 & 0.0020 & 1.784 & 0.035 & 0.0741 & 0.0012 & 0.581 & 1037 & 11 & 1044 & 33 & 1 \\
\hline
\end{tabular}

Notes : $\quad$ 1. Uncertainties given at the one $\sigma$ level.

2. Error in TEMORA reference zircon calibration was $0.44 \%$ for the analytical session.

( not included in above errors but required when comparing ${ }^{206} \mathrm{~Pb} /{ }^{238} \mathrm{U}$ data from different mounts)

3. $\mathrm{f}_{206} \%$ denotes the percentage of ${ }^{206} \mathrm{~Pb}$ that is common $\mathrm{Pb}$.

4. Correction for common $\mathrm{Pb}$ made using the measured ${ }^{204} \mathrm{~Pb} /{ }^{206} \mathrm{~Pb}$ ratio.

5. For $\%$ Disc, $0 \%$ denotes a concordant analysis. 
Table 9. SHRIMP U-Pb data for zircon grains from sample MBQ.

\begin{tabular}{|c|c|c|c|c|c|c|c|c|c|c|c|c|c|c|c|c|c|c|c|c|c|c|}
\hline \multirow[b]{2}{*}{$\begin{array}{c}\text { Grain. } \\
\text { spot }\end{array}$} & \multirow[b]{2}{*}{$\begin{array}{c}U \\
(\mathrm{ppm})\end{array}$} & \multirow[b]{2}{*}{$\begin{array}{l}\text { Th } \\
(\mathrm{ppm})\end{array}$} & \multirow[b]{2}{*}{$T h / U$} & \multirow[b]{2}{*}{$\begin{array}{l}{ }^{206} \mathrm{~Pb}^{*} \\
(\mathrm{ppm})\end{array}$} & \multirow[b]{2}{*}{${ }^{204} \mathrm{~Pb} /$} & \multirow[b]{2}{*}{$\begin{array}{l}f_{206} \\
\%\end{array}$} & & Total F & atios & & & & adiogen & Ratios & & & & & & e (Ma) & & \\
\hline & & & & & & & $\begin{array}{l}{ }^{238} \mathrm{U} / \\
{ }^{206} \mathrm{~Pb}\end{array}$ & \pm & $\begin{array}{l}{ }^{207} \mathrm{~Pb} / \\
{ }^{206} \mathrm{~Pb}\end{array}$ & \pm & $\begin{array}{c}{ }^{206} \mathrm{~Pb} / \\
{ }^{238} \mathrm{U}\end{array}$ & \pm & $\begin{array}{l}{ }^{207} \mathrm{~Pb} / \\
{ }^{235} \mathrm{U}\end{array}$ & \pm & $\begin{array}{l}{ }^{207} \mathrm{~Pb} / \\
{ }^{206} \mathrm{~Pb}\end{array}$ & \pm & $\rho$ & $\begin{array}{l}{ }^{206} \mathrm{~Pb} / \\
{ }^{238} \mathrm{U}\end{array}$ & \pm & $\begin{array}{l}{ }^{207} \mathrm{~Pb} / \\
{ }^{206} \mathrm{~Pb}\end{array}$ & \pm & $\begin{array}{c}\% \\
\text { Disc }\end{array}$ \\
\hline 1.1 & 83 & 70 & 0.84 & 8.5 & - & $<0.01$ & 8.436 & 0.124 & 0.0629 & 0.0012 & 0.1185 & 0.0017 & 1.028 & 0.025 & 0.0629 & 0.0012 & 0.606 & 722 & 10 & 705 & 41 & -2 \\
\hline 2.1 & 352 & 96 & 0.27 & 53.8 & - & $<0.01$ & 5.620 & 0.062 & 0.0761 & 0.0005 & 0.1780 & 0.0020 & 1.876 & 0.025 & 0.0764 & 0.0006 & 0.833 & 1056 & 11 & 1107 & 15 & 5 \\
\hline 3.1 & 241 & 143 & 0.60 & 20.6 & 0.000082 & $<0.01$ & 10.042 & 0.119 & 0.0594 & 0.0007 & 0.0997 & 0.0012 & & & & & & 613 & 7 & & & \\
\hline 4.1 & 491 & 152 & 0.31 & 61.8 & 0.000016 & 0.03 & 6.829 & 0.074 & 0.0697 & 0.0005 & 0.1464 & 0.0016 & 1.403 & 0.018 & 0.0695 & 0.0005 & 0.839 & 881 & 9 & 914 & 14 & 4 \\
\hline 5.1 & 568 & 66 & 0.12 & 51.8 & 0.000010 & 0.19 & 9.419 & 0.102 & 0.0628 & 0.0005 & 0.1060 & 0.0012 & & & & & & 649 & 7 & & & \\
\hline 6.1 & 451 & 4 & 0.01 & 35.8 & 0.000007 & 0.07 & 10.834 & 0.119 & 0.0596 & 0.0006 & 0.0922 & 0.0010 & & & & & & 569 & 6 & & & \\
\hline 7.1 & 904 & 250 & 0.28 & 93.9 & - & $<0.01$ & 8.266 & 0.086 & 0.0645 & 0.0004 & 0.1210 & 0.0013 & 1.076 & 0.013 & 0.0645 & 0.0004 & 0.887 & 736 & 7 & 758 & 11 & 3 \\
\hline 8.1 & 568 & 289 & 0.51 & 70.4 & 0.000018 & 0.03 & 6.928 & 0.074 & 0.0696 & 0.0004 & 0.1443 & 0.0015 & 1.379 & 0.018 & 0.0693 & 0.0005 & 0.844 & 869 & 9 & 909 & 14 & 4 \\
\hline 9.1 & 70 & 94 & 1.34 & 7.4 & - & $<0.01$ & 8.092 & 0.122 & 0.0644 & 0.0013 & 0.1237 & 0.0019 & 1.115 & 0.030 & 0.0653 & 0.0014 & 0.567 & 752 & 11 & 786 & 46 & 4 \\
\hline 10.1 & analysis $n$ & not compl & & & & & & & & & & & & & & & & & & & & \\
\hline 11.1 & 492 & 125 & 0.25 & 43.6 & 0.000015 & 0.08 & 9.691 & 0.106 & 0.0614 & 0.0005 & 0.1031 & 0.0012 & & & & & & 633 & 7 & & & \\
\hline 12.1 & 40 & 32 & 0.82 & 3.1 & 0.000311 & 0.44 & 11.005 & 0.205 & 0.0624 & 0.0019 & 0.0905 & 0.0017 & & & & & & 558 & 10 & & & \\
\hline 13.1 & 539 & 218 & 0.40 & 278.6 & 0.000006 & 0.01 & 1.663 & 0.018 & 0.2760 & 0.0006 & 0.6014 & 0.0063 & 22.885 & 0.246 & 0.2760 & 0.0006 & 0.979 & 3036 & 26 & 3340 & 3 & 9 \\
\hline 14.1 & 604 & 34 & 0.06 & 83.4 & 0.000020 & 0.03 & 6.223 & 0.066 & 0.0737 & 0.0004 & 0.1607 & 0.0017 & 1.625 & 0.020 & 0.0734 & 0.0005 & 0.856 & 960 & 9 & 1025 & 13 & 6 \\
\hline 15.1 & 124 & 109 & 0.88 & 16.1 & 0.000029 & 0.05 & 6.601 & 0.084 & 0.0716 & 0.0009 & 0.1514 & 0.0019 & 1.487 & 0.027 & 0.0712 & 0.0009 & 0.706 & 909 & 11 & 964 & 26 & 6 \\
\hline 16.1 & 3201 & 237 & 0.07 & 507.8 & 0.000074 & 0.11 & 5.415 & 0.055 & 0.1426 & 0.0004 & 0.1845 & 0.0019 & 3.602 & 0.038 & 0.1416 & 0.0004 & 0.957 & 1091 & 10 & 2247 & 5 & 51 \\
\hline 17.1 & 174 & 115 & 0.66 & 25.7 & - & $<0.01$ & 5.829 & 0.069 & 0.0734 & 0.0007 & 0.1716 & 0.0020 & 1.746 & 0.027 & 0.0738 & 0.0007 & 0.766 & 1021 & 11 & 1035 & 20 & 1 \\
\hline 18.1 & 795 & 63 & 0.08 & 59.9 & 0.000003 & $<0.01$ & 11.404 & 0.121 & 0.0583 & 0.0004 & 0.0877 & 0.0009 & & & & & & 542 & 6 & & & \\
\hline 19.1 & 113 & 119 & 1.06 & 45.9 & 0.000011 & 0.02 & 2.107 & 0.027 & 0.1594 & 0.0011 & 0.4744 & 0.0060 & 10.420 & 0.150 & 0.1593 & 0.0011 & 0.882 & 2503 & 26 & 2448 & 12 & -2 \\
\hline 20.1 & 899 & 35 & 0.04 & 312.9 & 0.000002 & $<0.01$ & 2.468 & 0.026 & 0.1563 & 0.0003 & 0.4051 & 0.0042 & 8.729 & 0.092 & 0.1563 & 0.0003 & 0.981 & 2193 & 19 & 2416 & 4 & 9 \\
\hline 21.1 & 1076 & 275 & 0.26 & 79.6 & 0.000006 & 0.07 & 11.612 & 0.121 & 0.0586 & 0.0004 & 0.0861 & 0.0009 & & & & & & 532 & 5 & & & \\
\hline 22.1 & 1355 & 931 & 0.69 & 338.4 & 0.000001 & $<0.01$ & 3.438 & 0.035 & 0.1404 & 0.0011 & 0.2908 & 0.0030 & 5.628 & 0.073 & 0.1404 & 0.0011 & 0.791 & 1646 & 15 & 2232 & 14 & 26 \\
\hline 23.1 & 433 & 223 & 0.52 & 37.7 & 0.000144 & 0.32 & 9.853 & 0.108 & 0.0631 & 0.0005 & 0.1012 & 0.0011 & & & & & & 621 & 7 & & & \\
\hline 24.1 & 186 & 125 & 0.67 & 20.7 & - & $<0.01$ & 7.737 & 0.093 & 0.0661 & 0.0008 & 0.1293 & 0.0016 & 1.178 & 0.020 & 0.0661 & 0.0008 & 0.725 & 784 & 9 & 810 & 24 & 3 \\
\hline 25.1 & 45 & 26 & 0.57 & 3.8 & 0.000121 & $<0.01$ & 10.190 & 0.177 & 0.0575 & 0.0016 & 0.0984 & 0.0018 & & & & & & 605 & 10 & & & \\
\hline 26.1 & 352 & 176 & 0.50 & 27.0 & 0.000017 & 0.14 & 11.213 & 0.126 & 0.0597 & 0.0006 & 0.0891 & 0.0010 & & & & & & 550 & 6 & & & \\
\hline 27.1 & 1482 & 330 & 0.22 & 157.8 & 0.000074 & 0.13 & 8.068 & 0.083 & 0.0697 & 0.0003 & 0.1238 & 0.0013 & 1.171 & 0.014 & 0.0686 & 0.0005 & 0.832 & 752 & 7 & 887 & 14 & 15 \\
\hline 28.1 & 115 & 250 & 2.17 & 13.1 & 0.000062 & 0.11 & 7.535 & 0.099 & 0.0655 & 0.0009 & 0.1326 & 0.0017 & 1.181 & 0.024 & 0.0646 & 0.0010 & 0.632 & 803 & 10 & 762 & 34 & -5 \\
\hline 29.1 & 829 & 180 & 0.22 & 112.0 & 0.000014 & 0.02 & 6.358 & 0.067 & 0.0705 & 0.0003 & 0.1572 & 0.0016 & 1.524 & 0.018 & 0.0703 & 0.0003 & 0.905 & 941 & 9 & 937 & 10 & -1 \\
\hline 30.1 & 425 & 200 & 0.47 & 65.0 & 0.000008 & 0.01 & 5.614 & 0.061 & 0.0760 & 0.0005 & 0.1781 & 0.0019 & 1.864 & 0.024 & 0.0759 & 0.0005 & 0.865 & 1057 & 11 & 1093 & 13 & 3 \\
\hline 31.1 & 504 & 397 & 0.79 & 68.6 & 0.000018 & 0.03 & 6.311 & 0.068 & 0.0711 & 0.0004 & 0.1584 & 0.0017 & 1.548 & 0.019 & 0.0709 & 0.0005 & 0.858 & 948 & 9 & 954 & 13 & 1 \\
\hline 32.1 & 803 & 228 & 0.28 & 122.7 & 0.000011 & 0.02 & 5.617 & 0.059 & 0.0753 & 0.0003 & 0.1780 & 0.0019 & 1.843 & 0.021 & 0.0751 & 0.0004 & 0.913 & 1056 & 10 & 1071 & 9 & 1 \\
\hline 33.1 & 7 & 4 & 0.54 & 0.8 & 0.000815 & 0.96 & 8.120 & 0.288 & 0.0719 & 0.0041 & 0.1220 & 0.0045 & & & & & & 742 & 26 & & & \\
\hline 34.1 & 1383 & 41 & 0.03 & 119.8 & - & 0.06 & 9.923 & 0.102 & 0.0609 & 0.0003 & 0.1007 & 0.0011 & & & & & & 619 & 6 & & & \\
\hline 35.1 & 555 & 208 & 0.38 & 127.0 & 0.000006 & 0.01 & 3.753 & 0.040 & 0.1127 & 0.0038 & 0.2665 & 0.0028 & 4.139 & 0.148 & 0.1127 & 0.0038 & 0.297 & 1523 & 14 & 1843 & 62 & 17 \\
\hline 36.1 & 5 & 2 & 0.32 & 0.6 & 0.003072 & 3.49 & 6.969 & 0.324 & 0.3630 & 0.1222 & 0.1385 & 0.0070 & 6.380 & 2.509 & 0.3341 & 0.1303 & 0.128 & 836 & 39 & 3636 & 597 & 77 \\
\hline 37.1 & 109 & 24 & 0.22 & 13.5 & 0.000143 & 0.25 & 6.942 & 0.091 & 0.0693 & 0.0009 & 0.1437 & 0.0019 & 1.331 & 0.037 & 0.0672 & 0.0016 & 0.481 & 865 & 11 & 844 & 50 & -3 \\
\hline 38.1 & 304 & 201 & 0.66 & 41.0 & 0.000043 & 0.07 & 6.364 & 0.071 & 0.0725 & 0.0006 & 0.1570 & 0.0018 & 1.556 & 0.022 & 0.0719 & 0.0006 & 0.780 & 940 & 10 & 983 & 18 & 4 \\
\hline 39.1 & 2931 & 315 & 0.11 & 243.8 & 0.000134 & 0.72 & 10.329 & 0.105 & 0.0655 & 0.0003 & 0.0961 & 0.0010 & & & & & & 592 & 6 & & & \\
\hline 40.1 & 422 & 154 & 0.37 & 37.2 & 0.000030 & $<0.01$ & 9.740 & 0.108 & 0.0605 & 0.0005 & 0.1027 & 0.0012 & & & & & & 630 & 7 & & & \\
\hline 41.1 & 398 & 163 & 0.41 & 51.1 & 0.000007 & 0.01 & 6.682 & 0.074 & 0.0687 & 0.0006 & 0.1496 & 0.0016 & 1.416 & 0.020 & 0.0686 & 0.0006 & 0.798 & 899 & 9 & 887 & 17 & -1 \\
\hline 42.1 & 277 & 97 & 0.35 & 36.2 & 0.000028 & 0.05 & 6.577 & 0.075 & 0.0700 & 0.0006 & 0.1520 & 0.0017 & 1.458 & 0.022 & 0.0696 & 0.0007 & 0.773 & 912 & 10 & 915 & 19 & 0 \\
\hline 43.1 & 806 & 95 & 0.12 & 89.6 & 0.000004 & 0.01 & 7.730 & 0.081 & 0.0701 & 0.0004 & 0.1294 & 0.0014 & 1.249 & 0.015 & 0.0700 & 0.0004 & 0.880 & 784 & 8 & 930 & 12 & 16 \\
\hline 44.1 & 144 & 103 & 0.72 & 15.6 & 0.000050 & 0.09 & 7.943 & 0.102 & 0.0659 & 0.0009 & 0.1258 & 0.0016 & 1.130 & 0.025 & 0.0652 & 0.0011 & 0.593 & 764 & 9 & 779 & 37 & 2 \\
\hline 45.1 & 299 & 118 & 0.40 & 28.3 & 0.000007 & 0.23 & 9.079 & 0.104 & 0.0638 & 0.0006 & 0.1099 & 0.0013 & & & & & & 672 & 7 & & & \\
\hline 46.1 & 627 & 388 & 0.62 & 64.7 & 0.000022 & 0.04 & 8.335 & 0.089 & 0.0639 & 0.0005 & 0.1199 & 0.0013 & 1.052 & 0.014 & 0.0636 & 0.0005 & 0.788 & 730 & 7 & 728 & 18 & 0 \\
\hline 47.1 & 172 & 58 & 0.34 & 15.0 & 0.000084 & $<0.01$ & 9.830 & 0.123 & 0.0598 & 0.0009 & 0.1018 & 0.0013 & & & & & & 625 & 8 & & & \\
\hline 48.1 & 92 & 129 & 1.40 & 7.0 & 0.000368 & 0.31 & 11.275 & 0.162 & 0.0610 & 0.0012 & 0.0884 & 0.0013 & & & & & & 546 & 8 & & & \\
\hline 49.1 & 179 & 207 & 1.16 & 14.8 & 0.000045 & 0.03 & 10.384 & 0.129 & 0.0599 & 0.0009 & 0.0963 & 0.0012 & & & & & & 593 & 7 & & & \\
\hline 50.1 & 37 & 24 & 0.65 & 5.6 & - & $<0.01$ & 5.730 & 0.101 & 0.0722 & 0.0015 & 0.1749 & 0.0031 & 1.786 & 0.061 & 0.0741 & 0.0021 & 0.523 & 1039 & 17 & 1043 & 58 & 0 \\
\hline 51.1 & 272 & 140 & 0.52 & 43.4 & - & $<0.01$ & 5.385 & 0.061 & 0.0741 & 0.0006 & 0.1857 & 0.0021 & 1.897 & 0.026 & 0.0741 & 0.0006 & 0.826 & 1098 & 11 & 1043 & 16 & -5 \\
\hline 52.1 & 1120 & 182 & 0.16 & 438.4 & 0.000022 & 0.03 & 2.196 & 0.023 & 0.2867 & 0.0004 & 0.4553 & 0.0047 & 17.987 & 0.187 & 0.2865 & 0.0004 & 0.990 & 2419 & 21 & 3399 & 2 & 29 \\
\hline 53.1 & 100 & 31 & 0.31 & 15.7 & 0.000097 & 0.16 & 5.472 & 0.074 & 0.0757 & 0.0010 & 0.1825 & 0.0025 & 1.870 & 0.040 & 0.0743 & 0.0013 & 0.626 & 1080 & 13 & 1051 & 34 & -3 \\
\hline 54.1 & 727 & 510 & 0.70 & 55.4 & 0.000015 & 0.04 & 11.268 & 0.121 & 0.0588 & 0.0005 & 0.0887 & 0.0010 & & & & & & 548 & 6 & & & \\
\hline 55.1 & 452 & 214 & 0.47 & 60.6 & 0.000027 & 0.05 & 6.415 & 0.070 & 0.0724 & 0.0005 & 0.1558 & 0.0017 & 1.548 & 0.021 & 0.0720 & 0.0006 & 0.809 & 933 & 9 & 987 & 16 & 5 \\
\hline 56.1 & 348 & 151 & 0.43 & 137.0 & 0.000011 & 0.02 & 2.186 & 0.024 & 0.1583 & 0.0008 & 0.4575 & 0.0050 & 9.980 & 0.120 & 0.1582 & 0.0008 & 0.902 & 2428 & 22 & 2437 & 9 & 0 \\
\hline 57.1 & 279 & 38 & 0.14 & 38.1 & 0.000021 & 0.04 & 6.308 & 0.072 & 0.0716 & 0.0006 & 0.1585 & 0.0018 & 1.559 & 0.022 & 0.0713 & 0.0006 & 0.796 & 948 & 10 & 967 & 18 & 2 \\
\hline 58.1 & 509 & 265 & 0.52 & 46.3 & 0.000021 & $<0.01$ & 9.438 & 0.103 & 0.0612 & 0.0005 & 0.1060 & 0.0012 & & & & & & 649 & 7 & & & \\
\hline 59.1 & 589 & 323 & 0.55 & 52.7 & - & $<0.01$ & 9.603 & 0.103 & 0.0605 & 0.0005 & 0.1042 & 0.0011 & & & & & & 639 & 7 & & & \\
\hline 60.1 & 1782 & 1109 & 0.62 & 130.4 & 0.000178 & 0.32 & 11.738 & 0.121 & 0.0605 & 0.0003 & 0.0849 & 0.0009 & & & & & & 525 & 5 & & & \\
\hline
\end{tabular}

Notes : $\quad$ 1. Uncertainties given at the one $\sigma$ level.

2. Error in Temora reference zircon calibration was $0.38 \%$ for the analytical session

(not included in above errors but required when comparing ${ }^{206} \mathrm{~Pb}^{238} \mathrm{U}$ data from different mounts)

3. $\mathrm{f}_{200} \%$ denotes the percentage of ${ }^{206} \mathrm{~Pb}$ that is common $\mathrm{Pb}$.

4. For areas older than $\sim 700 \mathrm{Ma}$ correction for common $\mathrm{Pb}$ made using the measured ${ }^{204} \mathrm{~Pb} /{ }^{206} \mathrm{~Pb}$ ratio

5. For areas younger than $\sim 700 \mathrm{Ma}$ correction for common $\mathrm{Pb}$ made using the measured ${ }^{238} \mathrm{U} /{ }^{206} \mathrm{~Pb}$ and ${ }^{207} \mathrm{~Pb} /{ }^{206} \mathrm{~Pb}$ ratios following Tera and Wasserburg (1972) as outlined in Williams (1998).

6. For $\%$ Disc, $0 \%$ denotes a concordant analysis. 
Table 10. SHRIMP U-Pb data for zircon grains from sample MS-5.

\begin{tabular}{|c|c|c|c|c|c|c|c|c|c|c|c|c|c|c|c|c|c|c|c|c|c|c|}
\hline \multirow[b]{2}{*}{$\begin{array}{c}\text { Grain. } \\
\text { spot }\end{array}$} & \multirow[b]{2}{*}{$\begin{array}{c}U \\
(\mathrm{ppm})\end{array}$} & \multirow[b]{2}{*}{$\begin{array}{c}\text { Th } \\
(\mathrm{ppm})\end{array}$} & \multirow[b]{2}{*}{$\mathrm{Th} / \mathrm{U}$} & & & & & Total F & atios & & & & adiogeni & Ratios & & & & & & e (Ma) & & \\
\hline & & & & $\begin{array}{l}{ }^{206} \mathrm{~Pb}^{\star} \\
(\mathrm{ppm})\end{array}$ & $\begin{array}{l}{ }^{204} \mathrm{~Pb} / \\
{ }^{206} \mathrm{~Pb}\end{array}$ & $\begin{array}{l}\mathrm{f}_{206} \\
\%\end{array}$ & $\begin{array}{l}{ }^{238} \mathrm{U} / \\
{ }^{206} \mathrm{~Pb}\end{array}$ & \pm & $\begin{array}{l}{ }^{207} \mathrm{~Pb} / \\
{ }^{206} \mathrm{~Pb}\end{array}$ & \pm & $\begin{array}{l}{ }^{206} \mathrm{~Pb} / \\
{ }^{238} \mathrm{U}\end{array}$ & \pm & $\begin{array}{c}{ }^{207} \mathrm{~Pb} / \\
{ }^{235} \mathrm{U}\end{array}$ & \pm & $\begin{array}{l}{ }^{207} \mathrm{~Pb} / \\
{ }^{2066} \mathrm{~Pb}\end{array}$ & \pm & $\rho$ & $\begin{array}{l}{ }^{206} \mathrm{~Pb} / \\
{ }^{238} \mathrm{U}\end{array}$ & \pm & $\begin{array}{l}{ }^{207} \mathrm{~Pb} / \\
{ }^{206} \mathrm{~Pb}\end{array}$ & \pm & $\begin{array}{c}\% \\
\text { Disc }\end{array}$ \\
\hline 1.1 & 768 & 112 & 0.15 & 99.5 & 0.000064 & 0.11 & 6.628 & 0.086 & 0.0755 & 0.0005 & 0.1507 & 0.0020 & 1.550 & 0.023 & 0.0746 & 0.0005 & 0.884 & 905 & 11 & 1057 & 14 & 14 \\
\hline 2.1 & 377 & 171 & 0.45 & 29.5 & 0.000048 & 0.14 & 10.999 & 0.122 & 0.0599 & 0.0005 & 0.0908 & 0.0010 & & & & & & 560 & 6 & & & \\
\hline 3.1 & 501 & 206 & 0.41 & 127.4 & 0.000007 & 0.01 & 3.378 & 0.037 & 0.1043 & 0.0003 & 0.2960 & 0.0032 & 4.254 & 0.048 & 0.1043 & 0.0003 & 0.957 & 1671 & 16 & 1701 & 6 & 2 \\
\hline 4.1 & 502 & 467 & 0.93 & 54.4 & 0.000039 & 0.07 & 7.928 & 0.086 & 0.0660 & 0.0004 & 0.1261 & 0.0014 & 1.137 & 0.015 & 0.0654 & 0.0005 & 0.823 & 765 & 8 & 788 & 16 & 3 \\
\hline 5.1 & 222 & 169 & 0.76 & 32.5 & 0.000026 & 0.04 & 5.884 & 0.068 & 0.0868 & 0.0012 & 0.1699 & 0.0020 & 2.024 & 0.036 & 0.0864 & 0.0012 & 0.645 & 1011 & 11 & 1348 & 26 & 25 \\
\hline 6.1 & 829 & 85 & 0.10 & 103.3 & 0.000017 & 0.03 & 6.895 & 0.072 & 0.0711 & 0.0003 & 0.1450 & 0.0015 & 1.416 & 0.016 & 0.0708 & 0.0003 & 0.918 & 873 & 9 & 953 & 9 & 8 \\
\hline 7.1 & 1379 & 11 & 0.01 & 98.1 & 0.000010 & 0.05 & 12.081 & 0.125 & 0.0580 & 0.0003 & 0.0827 & 0.0009 & & & & & & 512 & 5 & & & \\
\hline 8.1 & 1534 & 217 & 0.14 & 181.2 & 0.000287 & 0.49 & 7.274 & 0.075 & 0.0747 & 0.0002 & 0.1368 & 0.0014 & 1.331 & 0.016 & 0.0706 & 0.0004 & 0.854 & 827 & 8 & 945 & 13 & 13 \\
\hline 9.1 & 793 & 33 & 0.04 & 100.4 & - & $<0.01$ & 6.786 & 0.071 & 0.0698 & 0.0003 & 0.1474 & 0.0015 & 1.420 & 0.016 & 0.0699 & 0.0003 & 0.919 & 886 & 9 & 925 & 9 & 4 \\
\hline 10.1 & 67 & 27 & 0.40 & 9.4 & - & $<0.01$ & 6.094 & 0.088 & 0.0710 & 0.0012 & 0.1641 & 0.0024 & 1.606 & 0.036 & 0.0710 & 0.0012 & 0.639 & 980 & 13 & 957 & 36 & -2 \\
\hline 11.1 & 305 & 188 & 0.62 & 42.1 & 0.000015 & 0.03 & 6.223 & 0.070 & 0.0713 & 0.0005 & 0.1606 & 0.0018 & 1.575 & 0.021 & 0.0711 & 0.0005 & 0.832 & 960 & 10 & 961 & 15 & 0 \\
\hline 12.1 & 6621 & 888 & 0.13 & 881.2 & 0.000085 & 0.12 & 6.455 & 0.065 & 0.1470 & 0.0003 & 0.1547 & 0.0016 & 3.112 & 0.032 & 0.1459 & 0.0003 & 0.978 & 927 & 9 & 2298 & 4 & 60 \\
\hline 16.1 & 1257 & 33 & 0.03 & 404.5 & 0.000001 & 0.00 & 2.669 & 0.028 & 0.1308 & 0.0002 & 0.3746 & 0.0039 & 6.754 & 0.071 & 0.1307 & 0.0002 & 0.987 & 2051 & 18 & 2108 & 3 & 3 \\
\hline 15.1 & 131 & 76 & 0.58 & 31.2 & 0.000046 & 0.07 & 3.601 & 0.044 & 0.1015 & 0.0007 & 0.2775 & 0.0034 & 3.861 & 0.055 & 0.1009 & 0.0008 & 0.854 & 1579 & 17 & 1641 & 14 & 4 \\
\hline 14.1 & 443 & 265 & 0.60 & 102.4 & 0.000030 & 0.05 & 3.714 & 0.041 & 0.0995 & 0.0004 & 0.2691 & 0.0030 & 3.676 & 0.043 & 0.0991 & 0.0004 & 0.936 & 1536 & 15 & 1607 & 8 & 4 \\
\hline 13.1 & 1188 & 40 & 0.03 & 220.5 & 0.000020 & 0.03 & 4.627 & 0.048 & 0.0834 & 0.0002 & 0.2161 & 0.0022 & 2.476 & 0.027 & 0.0831 & 0.0003 & 0.960 & 1261 & 12 & 1272 & 6 & 1 \\
\hline 17.1 & 1881 & 343 & 0.18 & 480.5 & 0.000041 & 0.06 & 3.364 & 0.034 & 0.1526 & 0.0009 & 0.2971 & 0.0030 & 6.228 & 0.074 & 0.1520 & 0.0009 & 0.858 & 1677 & 15 & 2369 & 10 & 29 \\
\hline 18.1 & 502 & 165 & 0.33 & 96.1 & 0.000030 & 0.05 & 4.490 & 0.049 & 0.0896 & 0.0006 & 0.2226 & 0.0024 & 2.736 & 0.035 & 0.0891 & 0.0006 & 0.850 & 1296 & 13 & 1407 & 13 & 8 \\
\hline 19.1 & 160 & 149 & 0.94 & 25.9 & 0.000060 & 0.10 & 5.291 & 0.064 & 0.0780 & 0.0008 & 0.1888 & 0.0023 & 2.010 & 0.034 & 0.0772 & 0.0009 & 0.719 & 1115 & 12 & 1126 & 23 & 1 \\
\hline 20.1 & 181 & 183 & 1.01 & 19.3 & 0.000087 & 0.15 & 8.055 & 0.102 & 0.0777 & 0.0008 & 0.1240 & 0.0016 & 1.308 & 0.023 & 0.0765 & 0.0010 & 0.705 & 753 & 9 & 1108 & 25 & 32 \\
\hline 21.1 & 542 & 76 & 0.14 & 47.7 & 0.000049 & 0.05 & 9.751 & 0.187 & 0.0611 & 0.0007 & 0.1025 & 0.0020 & & & & & & 629 & 12 & & & \\
\hline 22.1 & 131 & 77 & 0.59 & 51.7 & 0.000017 & 0.02 & 2.179 & 0.026 & 0.1664 & 0.0007 & 0.4588 & 0.0056 & 10.514 & 0.136 & 0.1662 & 0.0007 & 0.939 & 2434 & 25 & 2520 & 7 & 3 \\
\hline 23.1 & 1192 & 218 & 0.18 & 151.3 & 0.000032 & 0.06 & 6.767 & 0.071 & 0.0708 & 0.0003 & 0.1477 & 0.0015 & 1.433 & 0.016 & 0.0704 & 0.0003 & 0.927 & 888 & 9 & 939 & 9 & 5 \\
\hline 24.1 & 492 & 130 & 0.26 & 73.3 & 0.000026 & 0.04 & 5.770 & 0.063 & 0.0782 & 0.0014 & 0.1732 & 0.0019 & 1.858 & 0.040 & 0.0778 & 0.0014 & 0.513 & 1030 & 10 & 1142 & 37 & 10 \\
\hline 25.1 & 974 & 65 & 0.07 & 104.7 & 0.000091 & 0.15 & 7.988 & 0.083 & 0.0901 & 0.0003 & 0.1250 & 0.0013 & 1.531 & 0.017 & 0.0888 & 0.0004 & 0.918 & 759 & 7 & 1400 & 9 & 46 \\
\hline 26.1 & 1394 & 347 & 0.25 & 206.1 & 0.000092 & 0.14 & 5.810 & 0.126 & 0.1081 & 0.0009 & 0.1719 & 0.0037 & 2.531 & 0.059 & 0.1068 & 0.0009 & 0.926 & 1022 & 20 & 1746 & 16 & 41 \\
\hline 27.1 & 135 & 96 & 0.71 & 51.3 & 0.000009 & 0.01 & 2.256 & 0.063 & 0.1635 & 0.0020 & 0.4433 & 0.0124 & 9.984 & 0.304 & 0.1634 & 0.0020 & 0.917 & 2365 & 55 & 2491 & 20 & 5 \\
\hline 28.1 & 252 & 149 & 0.59 & 59.4 & 0.000104 & 0.17 & 3.641 & 0.040 & 0.1012 & 0.0005 & 0.2742 & 0.0030 & 3.771 & 0.047 & 0.0998 & 0.0006 & 0.888 & 1562 & 15 & 1620 & 11 & 4 \\
\hline 29.1 & 1193 & 971 & 0.81 & 191.0 & 0.000055 & 0.09 & 5.366 & 0.108 & 0.0883 & 0.0011 & 0.1862 & 0.0037 & 2.247 & 0.054 & 0.0875 & 0.0011 & 0.836 & 1101 & 20 & 1372 & 25 & 20 \\
\hline 30.1 & 1022 & 222 & 0.22 & 127.8 & 0.000023 & 0.04 & 6.869 & 0.071 & 0.0696 & 0.0003 & 0.1455 & 0.0015 & 1.391 & 0.016 & 0.0693 & 0.0003 & 0.927 & 876 & 8 & 908 & 9 & 4 \\
\hline 31.1 & 175 & 88 & 0.50 & 84.2 & 0.000019 & 0.03 & 1.787 & 0.021 & 0.2091 & 0.0007 & 0.5596 & 0.0065 & 16.118 & 0.195 & 0.2089 & 0.0007 & 0.963 & 2865 & 27 & 2897 & 5 & 1 \\
\hline 32.1 & 391 & 93 & 0.24 & 176.9 & 0.000068 & 0.10 & 1.899 & 0.021 & 0.1655 & 0.0005 & 0.5295 & 0.0061 & 12.429 & 0.176 & 0.1703 & 0.0010 & 0.918 & 2739 & 26 & 2560 & 10 & -7 \\
\hline 33.1 & 1677 & 558 & 0.33 & 494.0 & 0.000077 & 0.10 & 2.917 & 0.036 & 0.1898 & 0.0019 & 0.3425 & 0.0043 & 8.917 & 0.143 & 0.1888 & 0.0019 & 0.780 & 1899 & 21 & 2732 & 16 & 31 \\
\hline 34.1 & 1052 & 267 & 0.25 & 268.4 & 0.000136 & 0.21 & 3.369 & 0.035 & 0.1246 & 0.0004 & 0.2962 & 0.0031 & 5.016 & 0.056 & 0.1228 & 0.0005 & 0.941 & 1673 & 16 & 1997 & 7 & 16 \\
\hline 35.1 & 1039 & 419 & 0.40 & 206.7 & 0.000145 & 0.24 & 4.318 & 0.073 & 0.0834 & 0.0007 & 0.2310 & 0.0039 & 2.590 & 0.052 & 0.0813 & 0.0009 & 0.845 & 1340 & 21 & 1229 & 21 & -9 \\
\hline 36.1 & 1435 & 561 & 0.39 & 164.0 & 0.000291 & 0.49 & 7.514 & 0.078 & 0.0804 & 0.0009 & 0.1324 & 0.0014 & 1.392 & 0.024 & 0.0762 & 0.0010 & 0.609 & 802 & 8 & 1102 & 27 & 27 \\
\hline 37.1 & 1341 & 108 & 0.08 & 227.0 & 0.000147 & 0.24 & 5.076 & 0.053 & 0.0960 & 0.0003 & 0.1965 & 0.0021 & 2.546 & 0.031 & 0.0940 & 0.0006 & 0.870 & 1157 & 11 & 1507 & 11 & 23 \\
\hline 43.1 & 582 & 685 & 1.18 & 135.3 & 0.000418 & 0.67 & 3.693 & 0.057 & 0.0993 & 0.0017 & 0.2690 & 0.0041 & 3.467 & 0.094 & 0.0935 & 0.0021 & 0.570 & 1536 & 21 & 1498 & 42 & -3 \\
\hline 42.1 & 709 & 350 & 0.49 & 102.6 & 0.000199 & 0.34 & 5.939 & 0.065 & 0.0762 & 0.0004 & 0.1678 & 0.0018 & 1.697 & 0.024 & 0.0734 & 0.0006 & 0.782 & 1000 & 10 & 1024 & 18 & 2 \\
\hline 38.1 & 329 & 195 & 0.59 & 40.9 & 0.000052 & 0.09 & 6.910 & 0.078 & 0.0689 & 0.0005 & 0.1446 & 0.0016 & 1.359 & 0.020 & 0.0682 & 0.0007 & 0.762 & 871 & 9 & 873 & 20 & 0 \\
\hline 39.1 & 3655 & 247 & 0.07 & 332.1 & 0.000652 & 1.79 & 9.455 & 0.096 & 0.0756 & 0.0005 & 0.1039 & 0.0011 & & & & & & 637 & 6 & & & \\
\hline 40.1 & 334 & 164 & 0.49 & 63.7 & - & $<0.01$ & 4.503 & 0.050 & 0.0910 & 0.0005 & 0.2221 & 0.0025 & 2.789 & 0.035 & 0.0911 & 0.0005 & 0.894 & 1293 & 13 & 1448 & 11 & 11 \\
\hline 41.1 & 440 & 241 & 0.55 & 56.0 & 0.000015 & 0.03 & 6.753 & 0.114 & 0.0711 & 0.0011 & 0.1480 & 0.0025 & 1.447 & 0.033 & 0.0709 & 0.0011 & 0.746 & 890 & 14 & 954 & 31 & 7 \\
\hline 44.1 & 483 & 338 & 0.70 & 79.7 & 0.000179 & 0.29 & 5.204 & 0.094 & 0.0990 & 0.0027 & 0.1916 & 0.0035 & 2.551 & 0.085 & 0.0966 & 0.0027 & 0.540 & 1130 & 19 & 1559 & 53 & 27 \\
\hline 45.1 & 379 & 72 & 0.19 & 58.1 & 0.000040 & 0.07 & 5.601 & 0.062 & 0.0749 & 0.0007 & 0.1784 & 0.0020 & 1.828 & 0.028 & 0.0743 & 0.0008 & 0.728 & 1058 & 11 & 1050 & 21 & -1 \\
\hline 46.1 & 474 & 185 & 0.39 & 79.3 & 0.000011 & 0.02 & 5.137 & 0.056 & 0.0817 & 0.0014 & 0.1946 & 0.0021 & 2.188 & 0.044 & 0.0815 & 0.0014 & 0.539 & 1146 & 11 & 1234 & 34 & 7 \\
\hline 47.1 & 867 & 1662 & 1.92 & 111.2 & 0.000046 & 0.08 & 6.695 & 0.106 & 0.0751 & 0.0009 & 0.1492 & 0.0024 & 1.531 & 0.031 & 0.0744 & 0.0010 & 0.774 & 897 & 13 & 1052 & 26 & 15 \\
\hline 48.1 & 1059 & 113 & 0.11 & 176.8 & -0.000003 & 0.00 & 5.146 & 0.053 & 0.0768 & 0.0003 & 0.1944 & 0.0020 & 2.069 & 0.027 & 0.0772 & 0.0004 & 0.943 & 1145 & 11 & 1126 & 9 & -2 \\
\hline 49.1 & 1016 & 556 & 0.55 & 73.7 & 0.000068 & 0.12 & 11.841 & 0.125 & 0.0590 & 0.0003 & 0.0844 & 0.0009 & & & & & & 522 & 5 & & & \\
\hline 50.1 & 157 & 85 & 0.55 & 49.3 & 001113 & 1.74 & 2.733 & 0.036 & 0.1220 & 0.0010 & 0.3595 & .0048 & 5.296 & 0.148 & 0.1069 & 0.0026 & 0.484 & 1980 & 23 & 1746 & 45 & -13 \\
\hline 51.1 & 310 & 88 & 0.28 & 54.0 & 0.000133 & 0.23 & 4.931 & 0.058 & 0.0720 & 0.0006 & 0.2044 & 0.0025 & 2.204 & 0.053 & 0.0782 & 0.0013 & 0.746 & 1199 & 13 & 1153 & 34 & -4 \\
\hline 52.1 & 944 & 117 & 0.12 & 114.1 & 0.000023 & 0.04 & 7.107 & 0.077 & 0.0716 & 0.0006 & 0.1406 & 0.0015 & 1.382 & 0.019 & 0.0712 & 0.0006 & 0.777 & 848 & 9 & 964 & 18 & 12 \\
\hline 53.1 & 1485 & 420 & 0.28 & 198.6 & 0.000256 & 0.41 & 6.422 & 0.068 & 0.0972 & 0.0006 & 0.1551 & 0.0016 & 2.002 & 0.027 & 0.0936 & 0.0008 & 0.780 & 929 & 9 & 1501 & 16 & 38 \\
\hline 56.1 & 165 & 37 & 0.23 & 68.4 & 000035 & 0.05 & 2.074 & 0.024 & 0.1861 & 0.0007 & 0.4819 & .0057 & 12.336 & 0.154 & 0.1857 & 0.0008 & 0.945 & 2535 & 25 & 2704 & 7 & 6 \\
\hline 57.1 & 250 & 232 & 0.93 & 46.5 & 0.000287 & 0.40 & 4.627 & 0.054 & 0.1797 & 0.0009 & 0.2153 & 0.0025 & 5.228 & 0.072 & 0.1761 & 0.0012 & 0.857 & 1257 & 13 & 2617 & 12 & 52 \\
\hline 58.1 & 653 & 379 & 0.58 & 131.5 & 0.000026 & 0.04 & 4.264 & 0.112 & 0.0971 & 0.0020 & 0.2344 & 0.0062 & 3.126 & 0.105 & 0.0967 & 0.0020 & 0.782 & 1358 & 32 & 1561 & 39 & 13 \\
\hline 59.1 & 78 & 91 & 1.17 & 19.0 & 0.000129 & 0.21 & 3.515 & 0.048 & 0.1006 & 0.0009 & 0.2839 & 0.0039 & 3.868 & 0.068 & 0.0988 & 0.0011 & 0.774 & 1611 & 19 & 1602 & 21 & -1 \\
\hline 61.1 & 1303 & 184 & 0.14 & 161.3 & 0.000031 & 0.05 & 6.941 & 0.072 & 0.0708 & 0.0002 & 0.1440 & 0.0015 & 1.397 & 0.016 & 0.0704 & 0.0003 & 0.919 & 867 & 8 & 939 & 9 & 8 \\
\hline 60.1 & 882 & 11 & 0.01 & 115.9 & 00016 & 0.03 & 6.539 & 0.076 & 0.0710 & .0003 & 0.1529 & 0.0018 & 1.492 & 0.018 & 0.0708 & 0.0003 & 0.937 & 917 & 10 & 951 & 9 & 4 \\
\hline 62.1 & 3635 & 2569 & 0.71 & 238.3 & 0.000316 & 3.32 & 13.101 & 0.138 & 0.0831 & 0.0004 & 0.0738 & 0.0008 & & & & & & 459 & 5 & & & \\
\hline 63.1 & 649 & 321 & 0.49 & 228.5 & 0.000054 & 0.08 & 2.438 & 0.031 & 0.1627 & 0.0007 & 0.4098 & 0.0052 & 9.152 & 0.122 & 0.1620 & 0.0007 & 0.946 & 2214 & 24 & 2477 & 7 & 11 \\
\hline 64.1 & 10074 & 2107 & 0.21 & 424.6 & 0.000609 & 2.11 & 20.382 & 0.206 & 0.0693 & 0.0002 & 0.0480 & 0.0005 & & & & & & 302 & 3 & & & \\
\hline 65.1 & 447 & 617 & 1.38 & 49.8 & 00036 & 0.06 & 7.701 & 0.083 & 0.0716 & & 0.1298 & 0.0014 & 1.271 & 0.016 & 0.0710 & 0.0005 & 0.838 & 787 & 8 & 958 & 14 & 18 \\
\hline 66.1 & 9664 & 4268 & 0.44 & 506.2 & 0.000189 & -1.31 & 16.400 & 0.513 & 0.0438 & 0.0098 & 0.0618 & 0.0021 & & & & & & 386 & 13 & & & \\
\hline 67.1 & 79 & 61 & 0.77 & 35.2 & 0.000043 & 0.06 & 1.921 & 0.026 & 0.1985 & 0.0010 & 0.5203 & 0.0071 & 14.201 & 0.210 & 0.1980 & 0.0011 & 0.927 & 2700 & 30 & 2810 & 9 & 4 \\
\hline 68.1 & 343 & 76 & 0.22 & 98.3 & 0.000012 & 0.02 & 2.996 & 0.033 & 0.1399 & 0.0007 & 0.3338 & 0.0037 & 6.429 & 0.078 & 0.1397 & 0.0007 & 0.910 & 1857 & 18 & 2224 & 9 & 17 \\
\hline
\end{tabular}

Notes:

1. Uncertainties given at the one $\sigma$ level.

2. Error in Temora reference zircon calibration was $0.38 \%$ for the analytical session.

(not included in above errors but required when comparing ${ }^{206} \mathrm{~Pb} /{ }^{238} \mathrm{U}$ data from different mounts).

3. $\mathrm{f}_{206} \%$ denotes the percentage of ${ }^{206} \mathrm{~Pb}$ that is common $\mathrm{Pb}$.

4. For areas older than $\sim 700 \mathrm{Ma}$ correction for common $\mathrm{Pb}$ made using the measured ${ }^{204} \mathrm{~Pb}{ }^{206} \mathrm{~Pb}$ ratio

5. For areas younger than $\sim 700 \mathrm{Ma}$ correction for common $\mathrm{Pb}$ made using the measured ${ }^{238} \mathrm{U} /{ }^{206} \mathrm{~Pb}$ and ${ }^{207} \mathrm{~Pb} /{ }^{206} \mathrm{~Pb}$ ratios

following Tera and Wasserburg (1972) as outlined in Williams (1998).

6. For $\%$ Disc, $0 \%$ denotes a concordant analysis. 
Table 11. SHRIMP U-Pb data for zircon grains from sample Thango.

\begin{tabular}{|c|c|c|c|c|c|c|c|c|c|c|c|c|c|c|c|c|c|c|c|c|c|c|}
\hline \multirow[b]{2}{*}{$\begin{array}{c}\text { Grain. } \\
\text { spot }\end{array}$} & \multirow[b]{2}{*}{$\begin{array}{c}U \\
(\mathrm{ppm})\end{array}$} & \multirow[b]{2}{*}{$\begin{array}{c}\text { Th } \\
\text { (ppm) }\end{array}$} & \multirow[b]{2}{*}{$\mathrm{Th} / \mathrm{U}$} & \multirow[b]{2}{*}{$\begin{array}{l}{ }^{206} \mathrm{~Pb}^{*} \\
(\mathrm{ppm})\end{array}$} & & & & Total F & atios & & & & adiogeni & Ratios & & & & & & e (Ma) & & \\
\hline & & & & & ${ }^{204} \mathrm{~Pb} /$ & $\begin{array}{l}f_{206} \\
\%\end{array}$ & $\begin{array}{l}{ }^{238} \mathrm{U} / \\
{ }^{206} \mathrm{~Pb}\end{array}$ & \pm & $\begin{array}{l}{ }^{207} \mathrm{~Pb} / \\
{ }^{206} \mathrm{~Pb}\end{array}$ & \pm & $\begin{array}{c}{ }^{206} \mathrm{~Pb} / \\
{ }^{238} \mathrm{U}\end{array}$ & \pm & $\begin{array}{l}{ }^{207} \mathrm{~Pb} / \\
{ }^{235} \mathrm{U}\end{array}$ & \pm & $\begin{array}{l}{ }^{207} \mathrm{~Pb} / \\
{ }^{206} \mathrm{~Pb}\end{array}$ & \pm & $\rho$ & ${ }^{206} \mathrm{~Pb} /$ & \pm & $\begin{array}{l}{ }^{207} \mathrm{~Pb} / \\
{ }^{206} \mathrm{~Pb}\end{array}$ & \pm & $\begin{array}{c}\% \\
\text { Disc }\end{array}$ \\
\hline 1.1 & 106 & 23 & 0.22 & 36 & 0.000112 & 0.16 & 2.502 & 0.038 & 0.1412 & 0.0013 & 0.3991 & 0.0061 & 7.691 & 0.146 & 0.1398 & 0.0016 & 0.810 & 2165 & 28 & 2224 & 19 & 3 \\
\hline 2.1 & 183 & 237 & 1.29 & 24 & - & $<0.01$ & 6.502 & 0.090 & 0.0716 & 0.0011 & 0.1539 & 0.0021 & 1.528 & 0.032 & 0.0720 & 0.0011 & 0.663 & 923 & 12 & 986 & 32 & 6 \\
\hline 3.1 & 564 & 91 & 0.16 & 73 & 0.000036 & 0.06 & 6.659 & 0.076 & 0.0725 & 0.0006 & 0.1501 & 0.0017 & 1.490 & 0.024 & 0.0720 & 0.0008 & 0.708 & 901 & 10 & 986 & 23 & 9 \\
\hline 4.1 & 189 & 79 & 0.42 & 24 & 0.000076 & 0.13 & 6.685 & 0.094 & 0.0712 & 0.0011 & 0.1494 & 0.0021 & 1.443 & 0.035 & 0.0701 & 0.0014 & 0.578 & 898 & 12 & 930 & 41 & 4 \\
\hline 5.1 & 34 & 31 & 0.93 & 5 & - & $<0.01$ & 6.145 & 0.160 & 0.0753 & 0.0026 & 0.1637 & 0.0043 & 1.815 & 0.109 & 0.0804 & 0.0043 & 0.441 & 977 & 24 & 1207 & 106 & 19 \\
\hline 6.1 & 1049 & 426 & 0.41 & 139 & 0.000016 & 0.03 & 6.501 & 0.072 & 0.0705 & 0.0005 & 0.1538 & 0.0017 & 1.490 & 0.020 & 0.0703 & 0.0005 & 0.839 & 922 & 9 & 937 & 15 & 2 \\
\hline 7.1 & 104 & 145 & 1.40 & 17 & 0.000266 & 0.45 & 5.308 & 0.074 & 0.0759 & 0.0011 & 0.1875 & 0.0026 & 1.864 & 0.059 & 0.0721 & 0.0020 & 0.447 & 1108 & 14 & 989 & 57 & -12 \\
\hline 8.1 & 729 & 103 & 0.14 & 58 & 0.000013 & 0.00 & 10.860 & 0.118 & 0.0590 & 0.0005 & 0.0921 & 0.0010 & & & & & & 568 & 6 & & & \\
\hline 9.1 & 335 & 211 & 0.63 & 87 & 0.000046 & 0.07 & 3.306 & 0.038 & 0.1073 & 0.0006 & 0.3022 & 0.0035 & 4.443 & 0.059 & 0.1066 & 0.0007 & 0.856 & 1702 & 17 & 1743 & 13 & 2 \\
\hline 10.1 & 126 & 82 & 0.65 & 17 & 0.000348 & 0.59 & 6.436 & 0.087 & 0.0769 & 0.0011 & 0.1545 & 0.0021 & 1.533 & 0.050 & 0.0720 & 0.0021 & 0.419 & 926 & 12 & 985 & 61 & 6 \\
\hline 11.1 & 1114 & 726 & 0.65 & 104 & 0.000202 & 1.50 & 9.173 & 0.097 & 0.0738 & 0.0006 & 0.1074 & 0.0012 & & & & & & 657 & 7 & & & \\
\hline 12.1 & 56 & 52 & 0.93 & 13 & 0.000201 & 0.33 & 3.834 & 0.063 & 0.0939 & 0.0016 & 0.2600 & 0.0043 & 3.265 & 0.127 & 0.0911 & 0.0032 & 0.429 & 1490 & 22 & 1448 & 67 & -3 \\
\hline 13.1 & 65 & 33 & 0.51 & 16 & 0.000039 & 0.06 & 3.450 & 0.054 & 0.1006 & 0.0013 & 0.2897 & 0.0046 & 3.998 & 0.085 & 0.1001 & 0.0014 & 0.743 & 1640 & 23 & 1626 & 26 & -1 \\
\hline 14.1 & 294 & 154 & 0.52 & 42 & 0.000010 & 0.02 & 6.012 & 0.072 & 0.0710 & 0.0007 & 0.1663 & 0.0020 & 1.625 & 0.025 & 0.0709 & 0.0007 & 0.763 & 992 & 11 & 954 & 21 & -4 \\
\hline 15.1 & 346 & 356 & 1.03 & 40 & 0.000032 & 0.06 & 7.401 & 0.086 & 0.0679 & 0.0008 & 0.1350 & 0.0016 & 1.256 & 0.022 & 0.0674 & 0.0008 & 0.677 & 817 & 9 & 851 & 26 & 4 \\
\hline 16.1 & 149 & 128 & 0.86 & 21 & 0.000239 & 0.41 & 6.167 & 0.080 & 0.0720 & 0.0009 & 0.1615 & 0.0021 & 1.527 & 0.042 & 0.0686 & 0.0017 & 0.476 & 965 & 12 & 887 & 50 & -9 \\
\hline 17.1 & 131 & 116 & 0.88 & 19 & 0.000246 & 0.42 & 6.022 & 0.080 & 0.0737 & 0.0012 & 0.1654 & 0.0022 & 1.600 & 0.055 & 0.0702 & 0.0022 & 0.393 & 986 & 12 & 933 & 65 & -6 \\
\hline 18.1 & 754 & 273 & 0.36 & 43 & 0.000250 & 0.54 & 15.170 & 0.167 & 0.0593 & 0.0006 & 0.0656 & 0.0007 & & & & & & 409 & 4 & & & \\
\hline 19.1 & 756 & 245 & 0.32 & 303 & - & $<0.01$ & 2.145 & 0.023 & 0.1636 & 0.0004 & 0.4663 & 0.0049 & 10.517 & 0.114 & 0.1636 & 0.0004 & 0.973 & 2467 & 22 & 2493 & 4 & 1 \\
\hline 20.1 & 287 & 366 & 1.27 & 22 & 0.000045 & 0.14 & 11.401 & 0.139 & 0.0595 & 0.0008 & 0.0876 & 0.0011 & & & & & & 541 & 6 & & & \\
\hline 21.1 & 108 & 91 & 0.84 & 15 & 0.000086 & 0.15 & 6.054 & 0.086 & 0.0740 & 0.0011 & 0.1650 & 0.0024 & 1.655 & 0.039 & 0.0728 & 0.0014 & 0.600 & 984 & 13 & 1008 & 39 & 2 \\
\hline 22.1 & 912 & 246 & 0.27 & 99 & 0.000033 & 0.06 & 7.955 & 0.084 & 0.0638 & 0.0004 & 0.1261 & 0.0014 & 1.154 & 0.022 & 0.0664 & 0.0007 & 0.856 & 766 & 8 & 818 & 23 & 6 \\
\hline 23.1 & 874 & 251 & 0.29 & 112 & 0.000047 & 0.08 & 6.718 & 0.072 & 0.0728 & 0.0004 & 0.1487 & 0.0016 & 1.479 & 0.019 & 0.0721 & 0.0005 & 0.826 & 894 & 9 & 989 & 15 & 10 \\
\hline 24.1 & 81 & 67 & 0.82 & 11 & 0.000192 & 0.33 & 6.161 & 0.095 & 0.0732 & 0.0014 & 0.1618 & 0.0025 & 1.572 & 0.054 & 0.0705 & 0.0022 & 0.454 & 967 & 14 & 942 & 63 & -3 \\
\hline 25.1 & 247 & 99 & 0.40 & 40 & 0.000023 & 0.04 & 5.273 & 0.064 & 0.0791 & 0.0008 & 0.1896 & 0.0023 & 2.060 & 0.033 & 0.0788 & 0.0008 & 0.749 & 1119 & 12 & 1168 & 21 & 4 \\
\hline 26.1 & 1954 & 1360 & 0.70 & 171 & 0.000394 & 5.01 & 9.827 & 0.101 & 0.1007 & 0.0004 & 0.0967 & 0.0010 & & & & & & 595 & 6 & & & \\
\hline 27.1 & 241 & 137 & 0.57 & 36 & 0.000159 & 0.27 & 5.808 & 0.070 & 0.0747 & 0.0008 & 0.1719 & 0.0023 & 1.738 & 0.059 & 0.0733 & 0.0018 & 0.806 & 1022 & 12 & 1023 & 50 & 0 \\
\hline 28.1 & 716 & 345 & 0.48 & 54 & 0.000091 & 0.41 & 11.297 & 0.123 & 0.0617 & 0.0005 & 0.0882 & 0.0010 & & & & & & 545 & 6 & & & \\
\hline 29.1 & 1364 & 199 & 0.15 & 99 & 0.000090 & 0.42 & 11.777 & 0.123 & 0.0612 & 0.0004 & 0.0846 & 0.0009 & & & & & & 523 & 5 & & & \\
\hline 30.1 & 201 & 65 & 0.32 & 29 & - & $<0.01$ & 6.037 & 0.076 & 0.0741 & 0.0009 & 0.1658 & 0.0021 & 1.709 & 0.030 & 0.0748 & 0.0009 & 0.709 & 989 & 11 & 1062 & 25 & 7 \\
\hline 31.1 & 161 & 94 & 0.58 & 38 & 0.000117 & 0.19 & 3.647 & 0.046 & 0.1000 & 0.0009 & 0.2737 & 0.0035 & 3.711 & 0.065 & 0.0984 & 0.0012 & 0.726 & 1559 & 18 & 1593 & 22 & 2 \\
\hline 32.1 & 2127 & 710 & 0.33 & 105 & 0.000536 & 2.43 & 17.337 & 0.179 & 0.0731 & 0.0004 & 0.0563 & 0.0006 & & & & & & 353 & 4 & & & \\
\hline 33.2 & 98 & 77 & 0.79 & 13 & 0.000175 & 0.30 & 6.679 & 0.101 & 0.0730 & 0.0017 & 0.1493 & 0.0023 & 1.452 & 0.059 & 0.0705 & 0.0026 & 0.379 & 897 & 13 & 944 & 77 & 5 \\
\hline 34.2 & 285 & 270 & 0.95 & 83 & 0.000044 & 0.07 & 2.959 & 0.034 & 0.1370 & 0.0008 & 0.3377 & 0.0039 & 6.351 & 0.083 & 0.1364 & 0.0008 & 0.885 & 1876 & 19 & 2182 & 11 & 14 \\
\hline 35.2 & 354 & 130 & 0.37 & 43 & 0.000002 & 0.00 & 7.139 & 0.082 & 0.0705 & 0.0007 & 0.1401 & 0.0016 & 1.362 & 0.021 & 0.0705 & 0.0007 & 0.752 & 845 & 9 & 943 & 21 & 10 \\
\hline 36.2 & 390 & 710 & 1.82 & 18 & 0.000541 & 2.08 & 18.651 & 0.229 & 0.0697 & 0.0011 & 0.0525 & 0.0007 & & & & & & 330 & 4 & & & \\
\hline 37.1 & 785 & 211 & 0.27 & 129 & - & $<0.01$ & 5.217 & 0.063 & 0.0758 & 0.0008 & 0.1918 & 0.0023 & 2.022 & 0.035 & 0.0765 & 0.0010 & 0.698 & 1131 & 13 & 1107 & 25 & -2 \\
\hline 38.2 & 124 & 49 & 0.39 & 19 & 0.000019 & 0.03 & 5.733 & 0.080 & 0.0766 & 0.0012 & 0.1744 & 0.0024 & 1.835 & 0.040 & 0.0763 & 0.0012 & 0.652 & 1036 & 13 & 1104 & 33 & 6 \\
\hline 39.2 & 237 & 348 & 1.47 & 17 & - & 0.21 & 11.856 & 0.155 & 0.0595 & 0.0011 & 0.0842 & 0.0011 & & & & & & 521 & 7 & & & \\
\hline 40.2 & 766 & 62 & 0.08 & 97 & 0.000040 & 0.07 & 6.763 & 0.073 & 0.0715 & 0.0005 & 0.1478 & 0.0016 & 1.444 & 0.019 & 0.0709 & 0.0005 & 0.813 & 888 & 9 & 954 & 16 & 7 \\
\hline 41.2 & 211 & 192 & 0.91 & 24 & - & $<0.01$ & 7.536 & 0.097 & 0.0717 & 0.0010 & 0.1327 & 0.0017 & 1.315 & 0.025 & 0.0719 & 0.0010 & 0.669 & 803 & 10 & 982 & 29 & 18 \\
\hline 42.2 & 216 & 64 & 0.30 & 29 & 0.000082 & 0.14 & 6.484 & 0.082 & 0.0730 & 0.0012 & 0.1540 & 0.0020 & 1.524 & 0.039 & 0.0718 & 0.0016 & 0.493 & 923 & 11 & 980 & 46 & 6 \\
\hline 43.1 & 24 & 7 & 0.30 & 5 & 0.001755 & 3.19 & 4.484 & 0.168 & 0.0784 & 0.0043 & 0.2218 & 0.0088 & 2.265 & 0.231 & 0.0741 & 0.0061 & 0.645 & 1291 & 46 & 1043 & 166 & -24 \\
\hline 44.2 & 163 & 180 & 1.10 & 63 & 0.000083 & 0.12 & 2.225 & 0.028 & 0.1610 & 0.0010 & 0.4489 & 0.0057 & 9.903 & 0.142 & 0.1600 & 0.0011 & 0.883 & 2390 & 25 & 2456 & 11 & 3 \\
\hline 45.2 & 260 & 115 & 0.44 & 35 & - & $<0.01$ & 6.455 & 0.078 & 0.0730 & 0.0008 & 0.1554 & 0.0019 & 1.614 & 0.032 & 0.0753 & 0.0012 & 0.613 & 931 & 11 & 1078 & 31 & 14 \\
\hline 46.2 & 716 & 403 & 0.56 & 84 & 0.000076 & 0.13 & 7.306 & 0.080 & 0.0723 & 0.0006 & 0.1367 & 0.0015 & 1.343 & 0.021 & 0.0713 & 0.0008 & 0.711 & 826 & 9 & 965 & 22 & 14 \\
\hline 47.2 & 164 & 90 & 0.55 & 36 & - & $<0.01$ & 3.860 & 0.051 & 0.1006 & 0.0011 & 0.2593 & 0.0034 & 3.615 & 0.064 & 0.1011 & 0.0012 & 0.746 & 1486 & 18 & 1645 & 22 & 10 \\
\hline 48.2 & 361 & 205 & 0.57 & 38 & 0.000082 & 0.55 & 8.053 & 0.097 & 0.0688 & 0.0008 & 0.1235 & 0.0015 & & & & & & 751 & 9 & & & \\
\hline 49.2 & 225 & 190 & 0.84 & 31 & 0.000088 & 0.15 & 6.312 & 0.078 & 0.0702 & 0.0009 & 0.1585 & 0.0022 & 1.544 & 0.073 & 0.0707 & 0.0026 & 0.803 & 949 & 12 & 947 & 76 & 0 \\
\hline 50.2 & 298 & 217 & 0.73 & 128 & 0.000012 & 0.02 & 1.993 & 0.023 & 0.1840 & 0.0008 & 0.5016 & 0.0058 & 12.714 & 0.157 & 0.1839 & 0.0008 & 0.939 & 2620 & 25 & 2688 & 7 & 3 \\
\hline 51.1 & 103 & 93 & 0.91 & 8 & 0.000402 & 0.16 & 11.604 & 0.180 & 0.0594 & 0.0015 & 0.0860 & 0.0014 & & & & & & 532 & 8 & & & \\
\hline 52.1 & 639 & 112 & 0.17 & 125 & 0.000043 & 0.07 & 4.392 & 0.048 & 0.0905 & 0.0005 & 0.2275 & 0.0025 & 2.822 & 0.035 & 0.0899 & 0.0006 & 0.867 & 1322 & 13 & 1424 & 12 & 7 \\
\hline 53.1 & 33 & 12 & 0.37 & 5 & - & $<0.01$ & 5.892 & 0.124 & 0.0775 & 0.0023 & 0.1713 & 0.0039 & 2.023 & 0.201 & 0.0856 & 0.0083 & 0.231 & 1019 & 22 & 1330 & 187 & 23 \\
\hline 54.1 & 333 & 196 & 0.59 & 57 & 0.000046 & 0.08 & 5.059 & 0.058 & 0.0846 & 0.0007 & 0.1975 & 0.0023 & 2.287 & 0.035 & 0.0840 & 0.0008 & 0.754 & 1162 & 12 & 1292 & 19 & 10 \\
\hline 55.1 & 4660 & 742 & 0.16 & 183 & 0.000661 & 1.98 & 21.823 & 0.224 & 0.0678 & 0.0003 & 0.0449 & 0.0005 & & & & & & 283 & 3 & & & \\
\hline 56.1 & 396 & 265 & 0.67 & 54 & 0.000019 & 0.03 & 6.310 & 0.072 & 0.0717 & 0.0007 & 0.1584 & 0.0018 & 1.561 & 0.024 & 0.0715 & 0.0007 & 0.751 & 948 & 10 & 971 & 20 & 2 \\
\hline 57.1 & 122 & 93 & 0.76 & 17 & 0.000115 & 0.20 & 6.274 & 0.088 & 0.0750 & 0.0021 & 0.1591 & 0.0023 & 1.610 & 0.062 & 0.0734 & 0.0026 & 0.368 & 952 & 13 & 1025 & 73 & 7 \\
\hline 58.1 & 66 & 111 & 1.69 & 5 & - & 0.87 & 12.148 & 0.220 & 0.0644 & 0.0020 & 0.0816 & 0.0015 & & & & & & 506 & 9 & & & \\
\hline 59.1 & 210 & 105 & 0.50 & 28 & - & $<0.01$ & 6.375 & 0.080 & 0.0729 & 0.0009 & 0.1569 & 0.0020 & 1.582 & 0.029 & 0.0731 & 0.0009 & 0.698 & 940 & 11 & 1018 & 26 & 8 \\
\hline 60.1 & 124 & 174 & 1.41 & 14 & 0.000260 & 0.46 & 7.427 & 0.103 & 0.0656 & 0.0012 & 0.1346 & 0.0025 & 1.207 & 0.117 & 0.0650 & 0.0054 & 0.826 & 814 & 14 & 776 & 174 & -5 \\
\hline 61.1 & 45 & 21 & 0.46 & 18 & 0.000169 & 0.24 & 2.178 & 0.038 & 0.1596 & 0.0018 & 0.4580 & 0.0080 & 9.945 & 0.226 & 0.1575 & 0.0023 & 0.771 & 2431 & 35 & 2429 & 25 & 0 \\
\hline 62.1 & 349 & 178 & 0.51 & 72 & 0.000030 & 0.05 & 4.165 & 0.048 & 0.0904 & 0.0006 & 0.2400 & 0.0028 & 2.978 & 0.043 & 0.0900 & 0.0008 & 0.805 & 1387 & 14 & 1425 & 16 & 3 \\
\hline 63.1 & 171 & 296 & 1.73 & 12 & - & 0.43 & 12.520 & 0.173 & 0.0606 & 0.0013 & 0.0795 & 0.0011 & & & & & & 493 & 7 & & & \\
\hline 64.1 & 372 & 71 & 0.19 & 35 & 0.000004 & 0.21 & 9.089 & 0.107 & 0.0636 & 0.0007 & 0.1098 & 0.0013 & & & & & & 672 & 8 & & & \\
\hline
\end{tabular}

Notes: $\quad$ 1. Uncertainties given at the one $\sigma$ level.

2. Error in Temora reference zircon calibration was $0.96 \%$ \& $1.33 \%$ for the two analytical sessions.

( not included in above errors but required when comparing ${ }^{206} \mathrm{~Pb} /{ }^{238} \mathrm{U}$ data from different mounts).

3. $\mathrm{f}_{206} \%$ denotes the percentage of ${ }^{206} \mathrm{~Pb}$ that is common $\mathrm{Pb}$.

4. For areas older than $\sim 800 \mathrm{Ma}$ correction for common $\mathrm{Pb}$ made using the measured ${ }^{204} \mathrm{~Pb} /{ }^{206} \mathrm{~Pb}$ ratio.

5. For areas younger than $\sim 800 \mathrm{Ma}$ correction for common $\mathrm{Pb}$ made using the measured ${ }^{238} \mathrm{U} /{ }^{206} \mathrm{~Pb}$ and ${ }^{207} \mathrm{~Pb} /{ }^{206} \mathrm{~Pb}$ ratios following Tera and Wasserburg (1972) as outlined in Williams (1998).

6. For $\%$ Disc, $0 \%$ denotes a concordant analysis. 
Data Repository: Myrow et al., Tera-Wasserburg and Wetherill concordia plots, and other plots. 


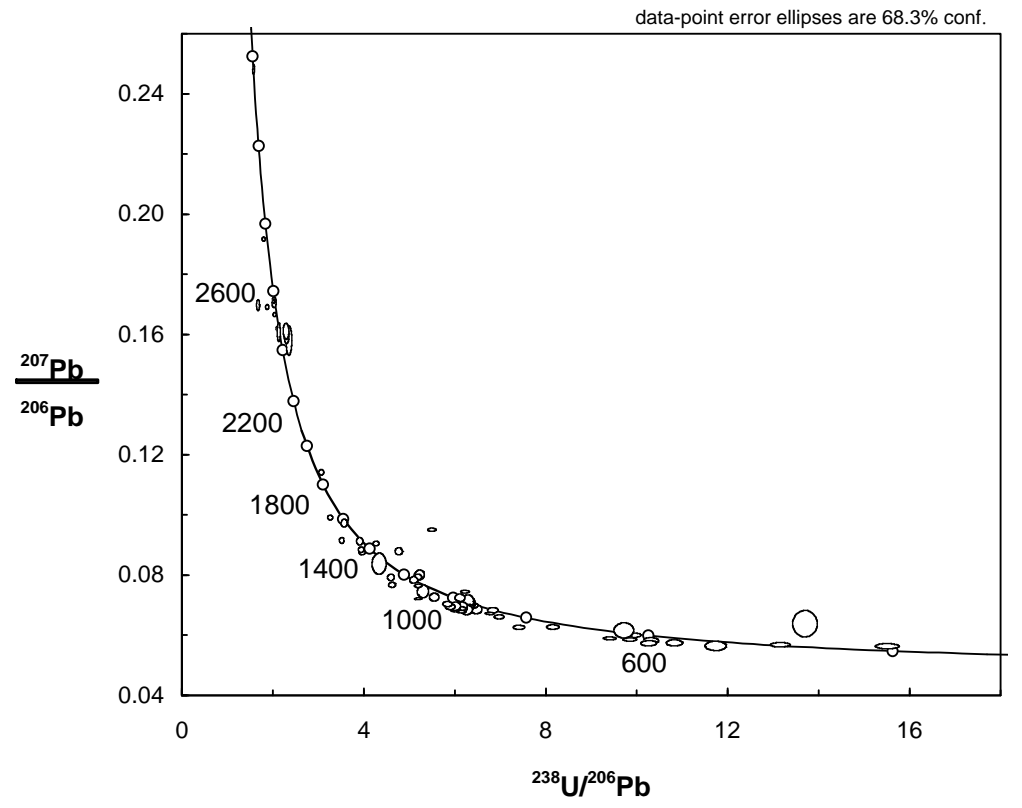

DR Fig.1a. Tera-Wasserburg concordia plot for all data from sample BATAL

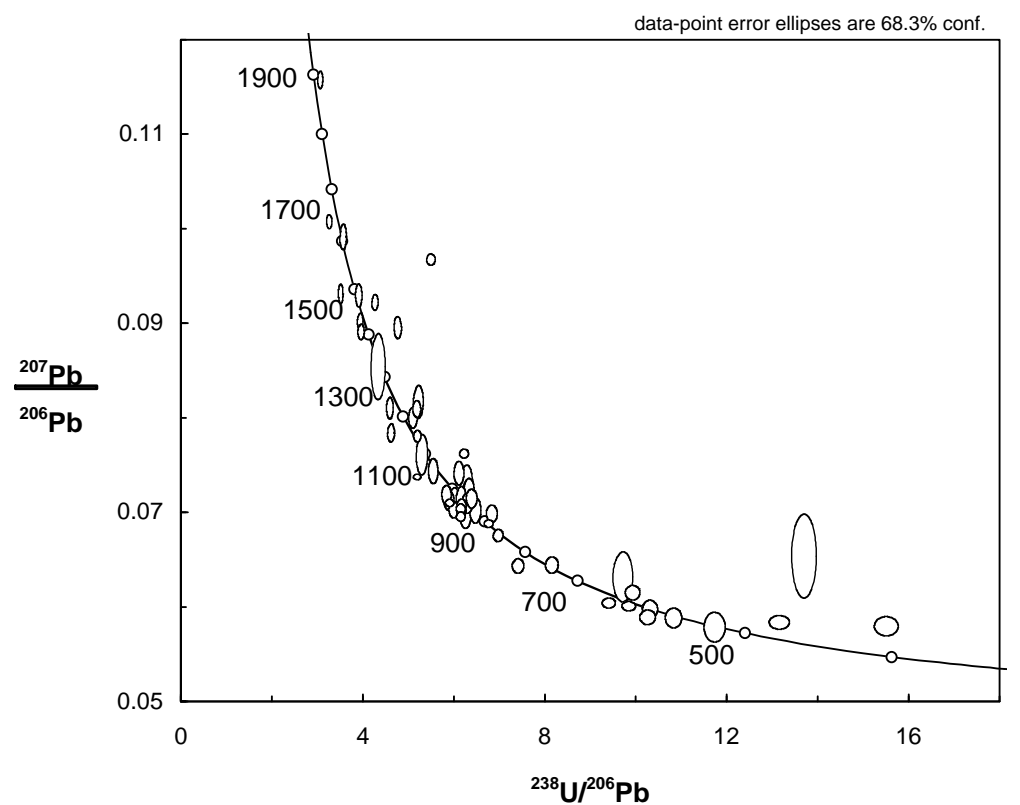

DR Fig.1b. Enlarged Tera-Wasserburg concordia plot for analyses $\leq 1900$ Ma from sample BATAL, highlighting the discordant nature of the three youngest analyses. 


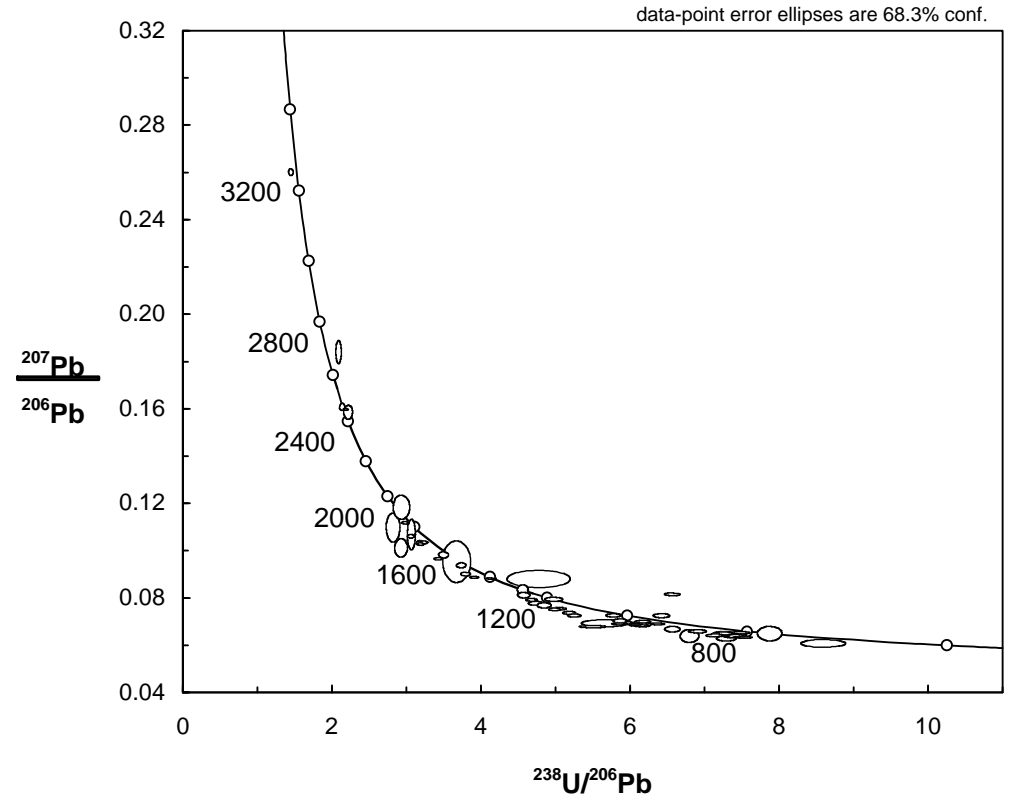

DR Fig.2a. Tera-Wasserburg concordia plot for all data from sample MS-2

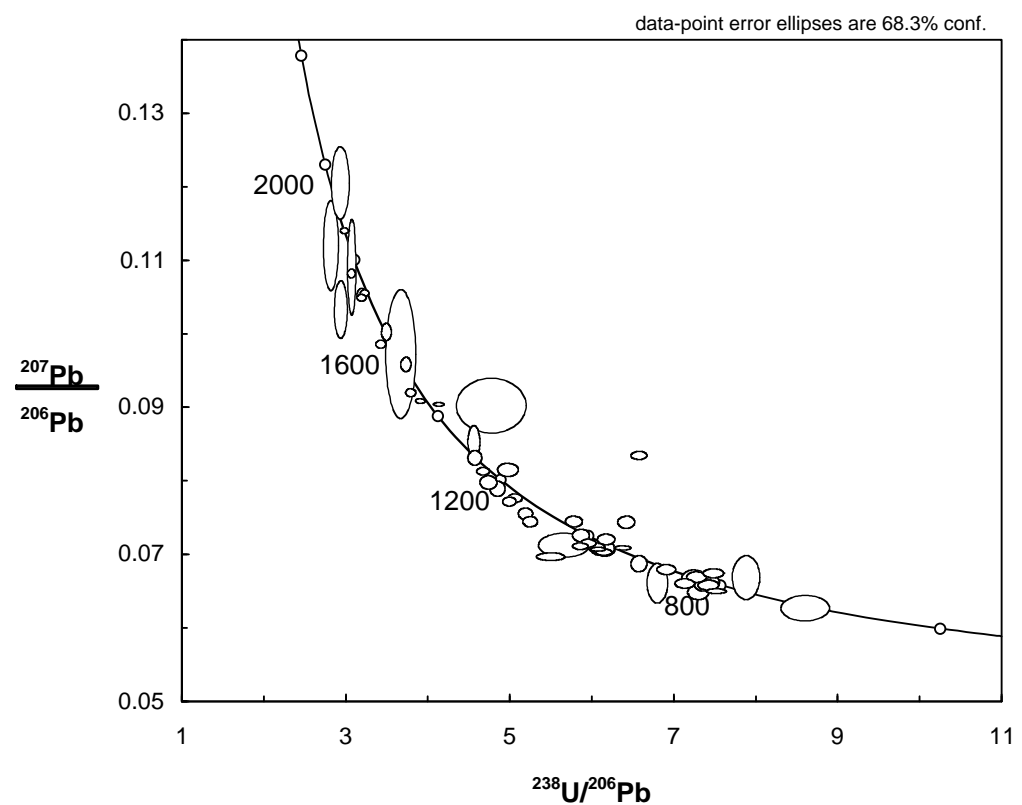

DR Fig.2b. Enlarged Tera-Wasserburg concordia plot for analyses $\leq 1900$ Ma from sample MS2. 


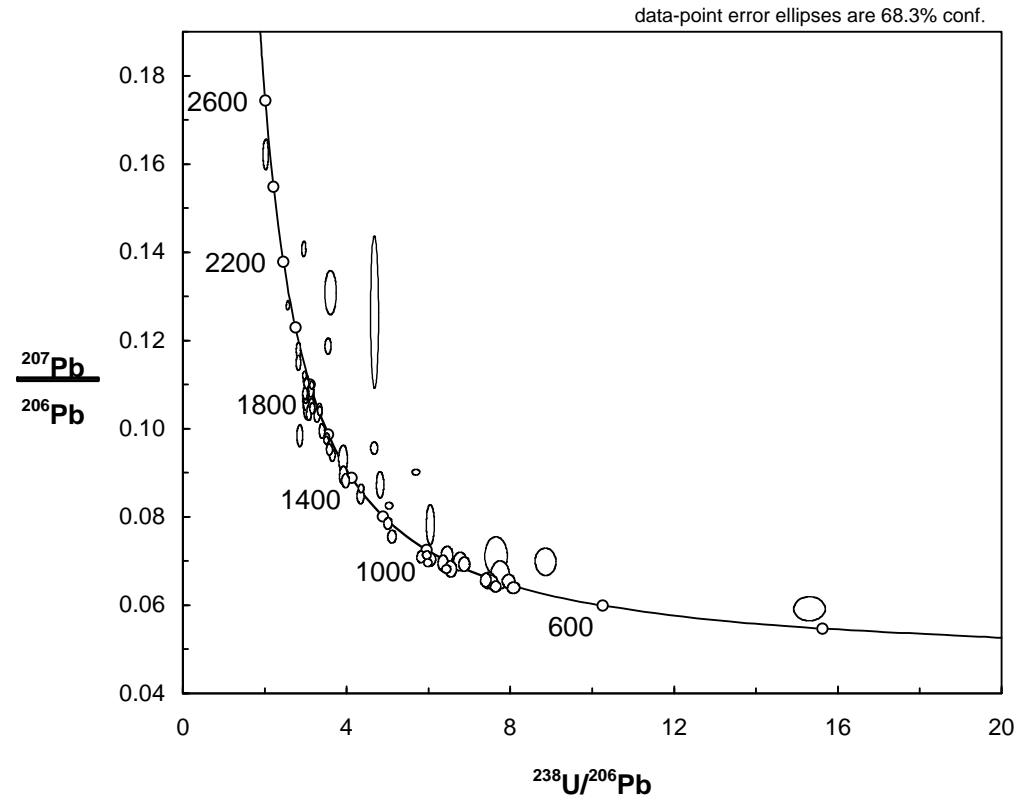

DR Fig.3a. Tera-Wasserburg concordia plot for all data from sample Tal

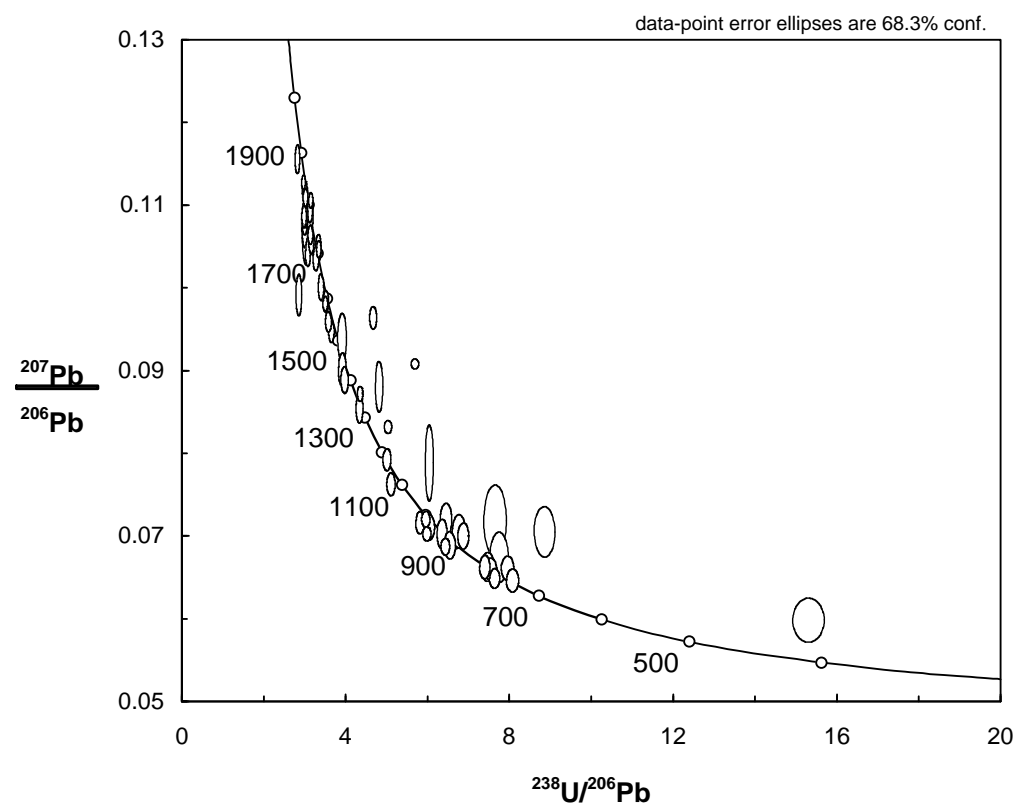

DR Fig.3b. Enlarged Tera-Wasserburg concordia plot for analyses $\leq 1900$ Ma from sample Tal. Note the two youngest analyses are significantly discordant. 


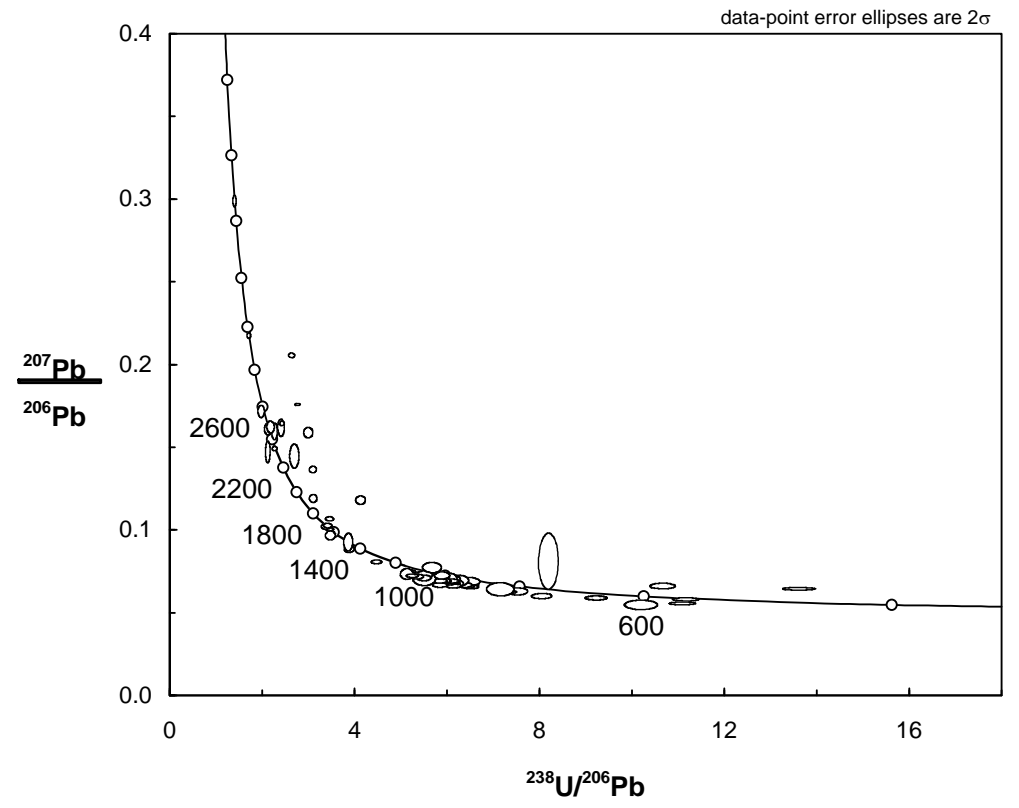

DR Fig.4a. Tera-Wasserburg concordia plot for all data from sample PV

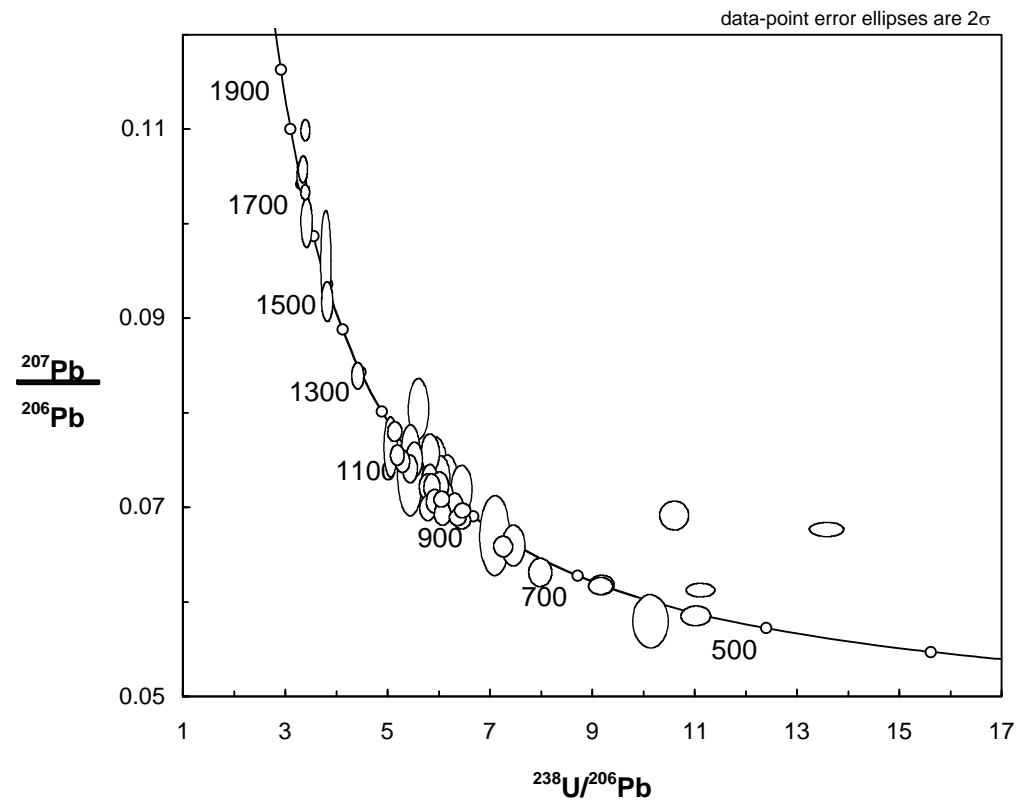

DR Fig.4b. Enlarged Tera-Wasserburg concordia plot for analyses $\leq 1900$ Ma from sample PV. 


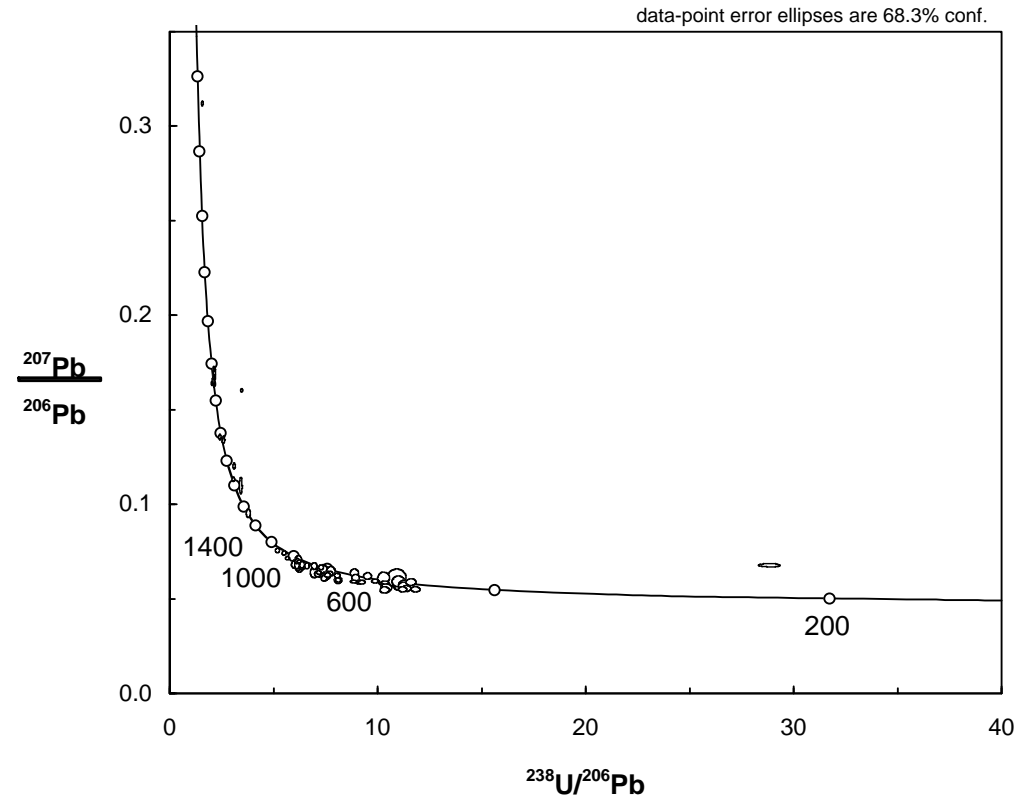

DR Fig.5a. Tera-Wasserburg concordia plot for all data from sample Tal-GKM

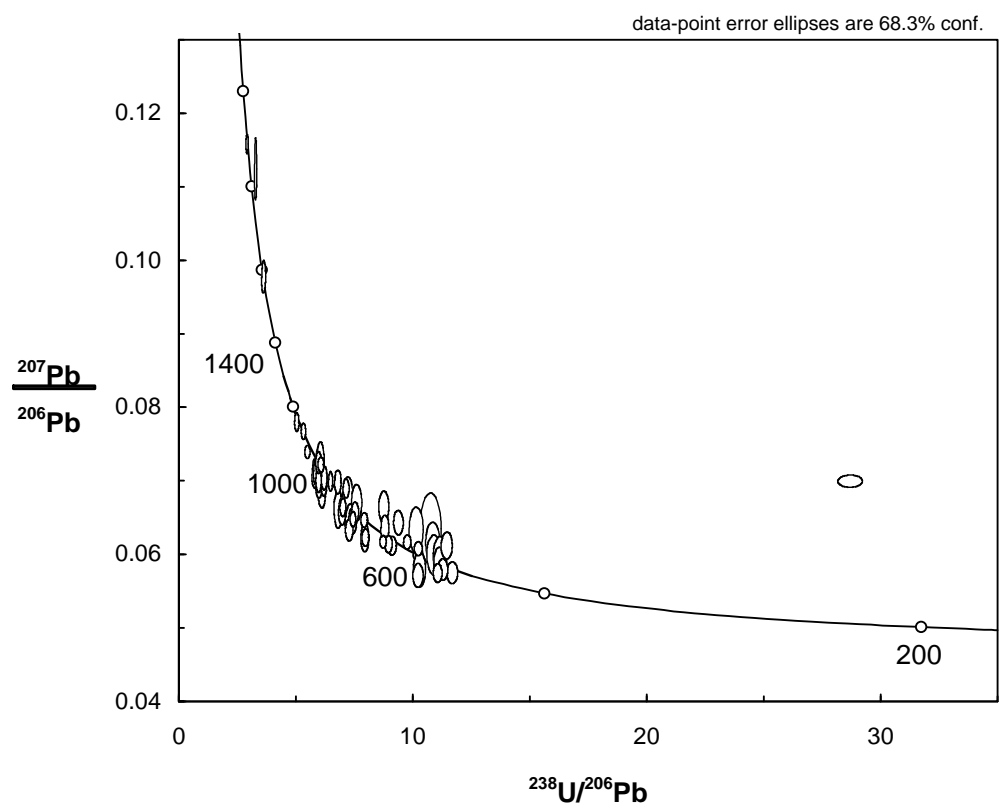

DR Fig.5b. Enlarged Tera-Wasserburg concordia plot for analyses $\leq 1900$ Ma from sample TalGKM. Note the significantly discordant analysis of grain 20. 


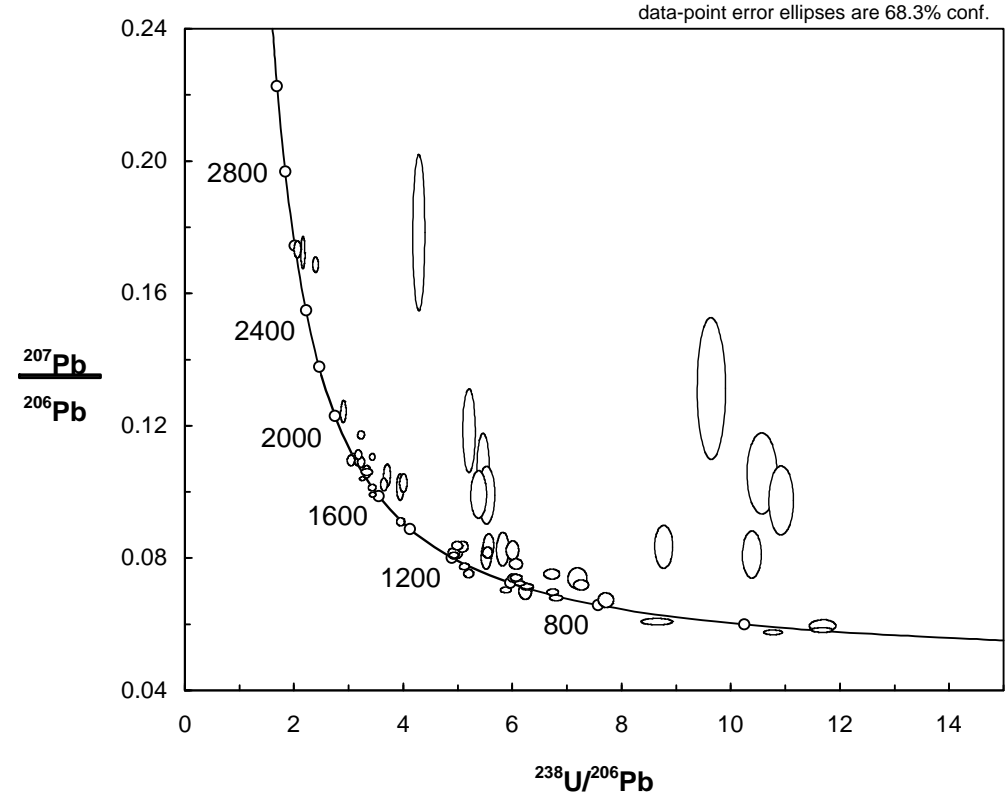

DR Fig.6a. Tera-Wasserburg concordia plot for all data from sample NY-11

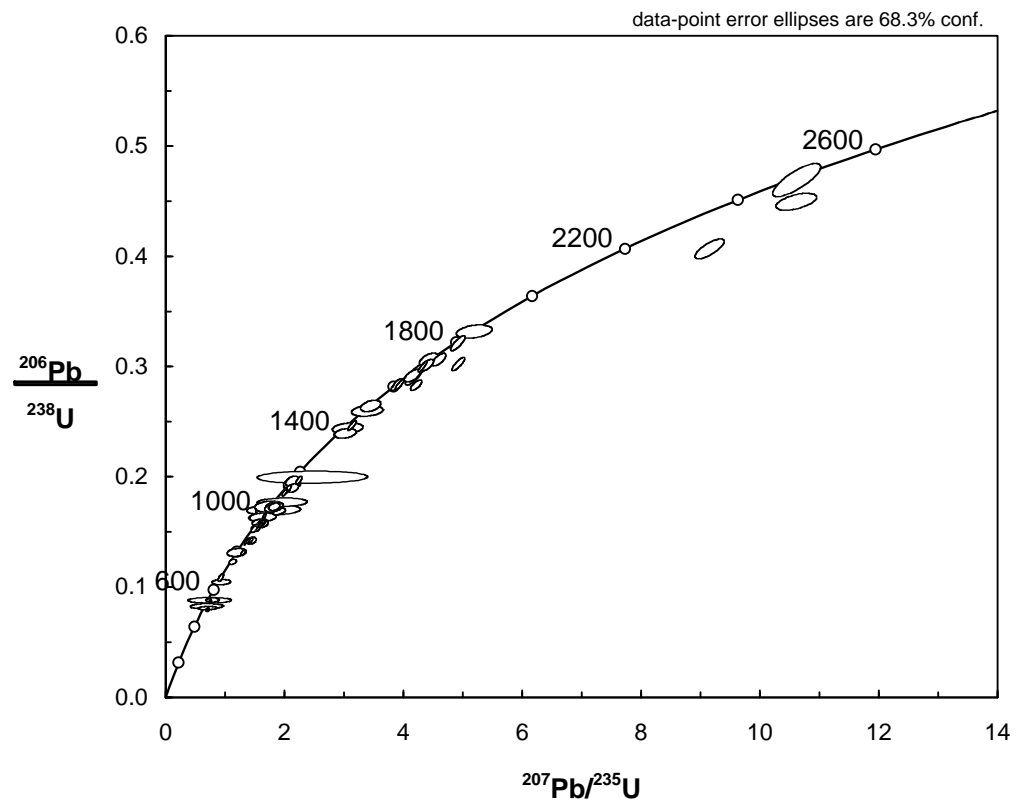

DR Fig.6b. Wetherill concordia plot for all analyses from sample NY-11. Note the general concordance of the ${ }^{204} \mathrm{~Pb}$ corrected data. 


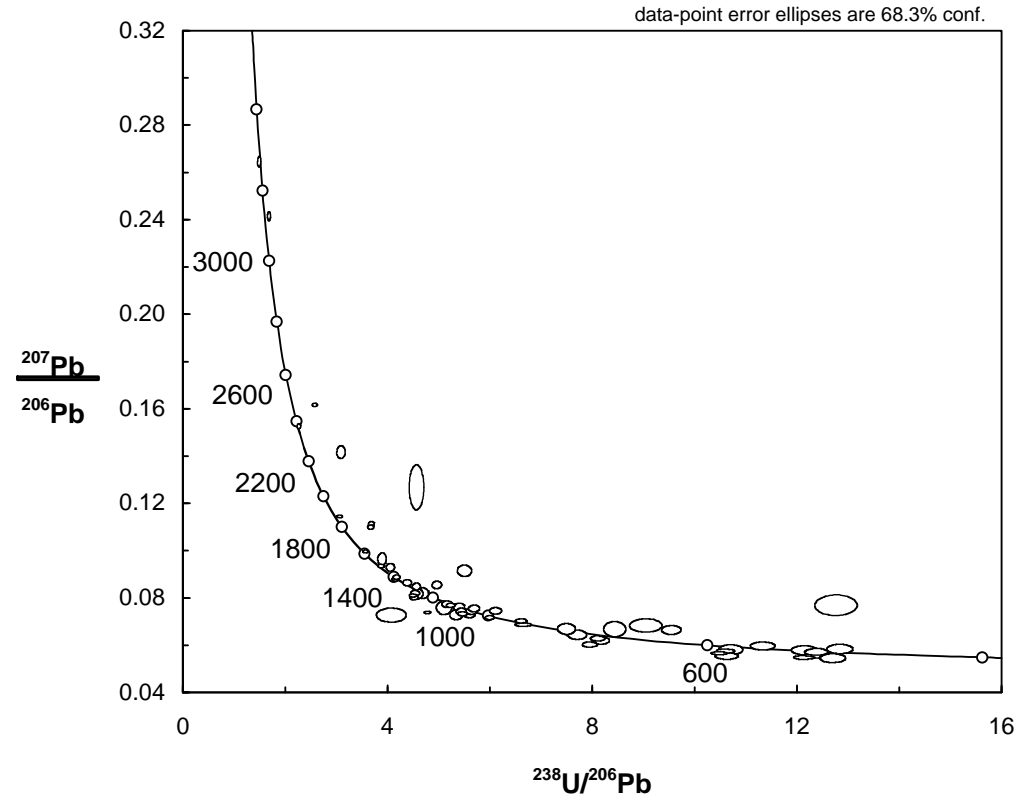

DR Fig.7a. Tera-Wasserburg concordia plot for all data from sample WL-270.

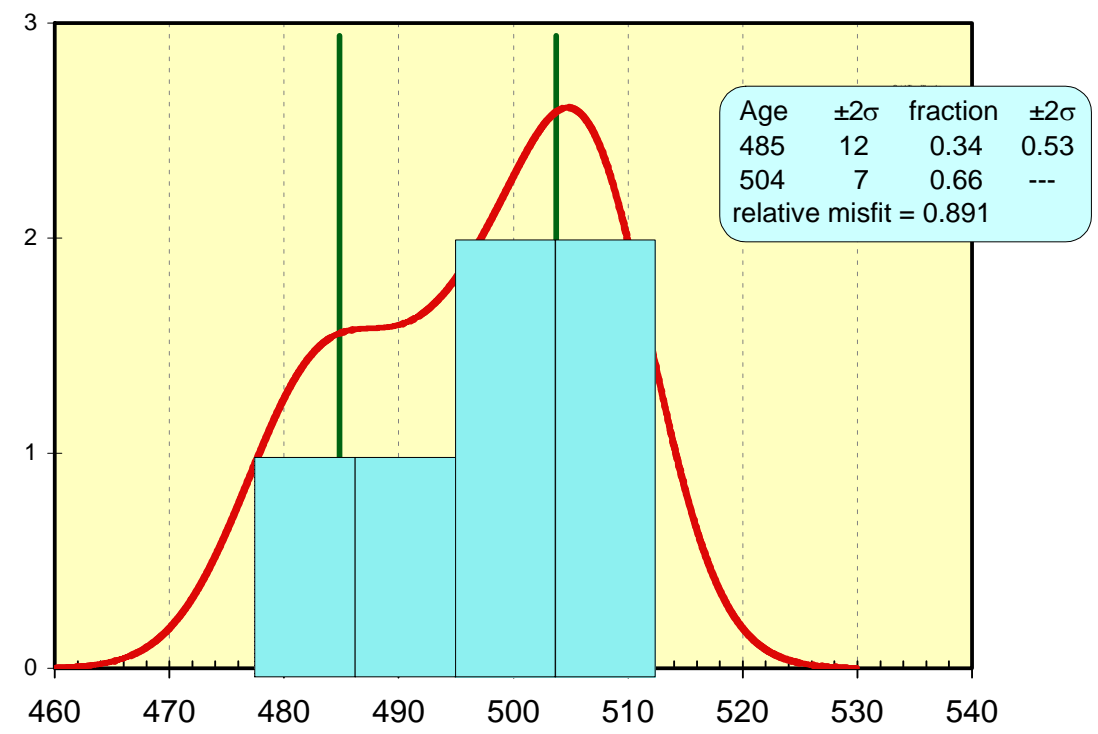

DR Fig.7b. Mixture modelling possibility for the 6 significant youngest analyses from sample WL-270 


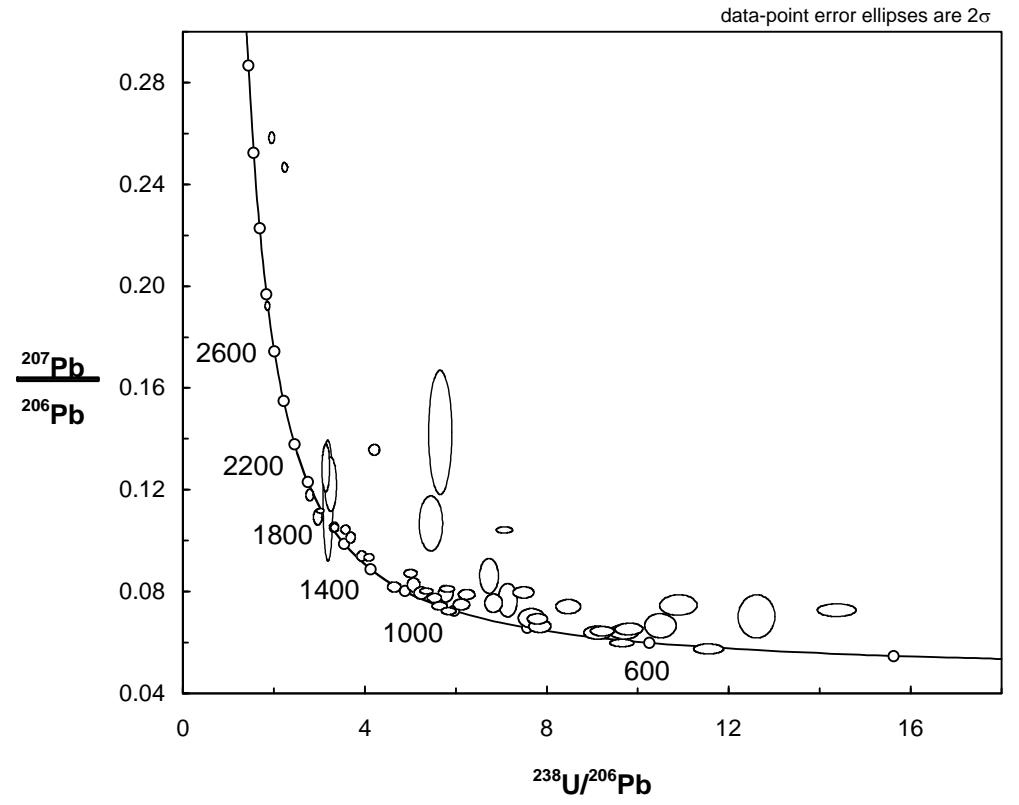

DR Fig.8a. Tera-Wasserburg concordia plot for all data from sample KU2

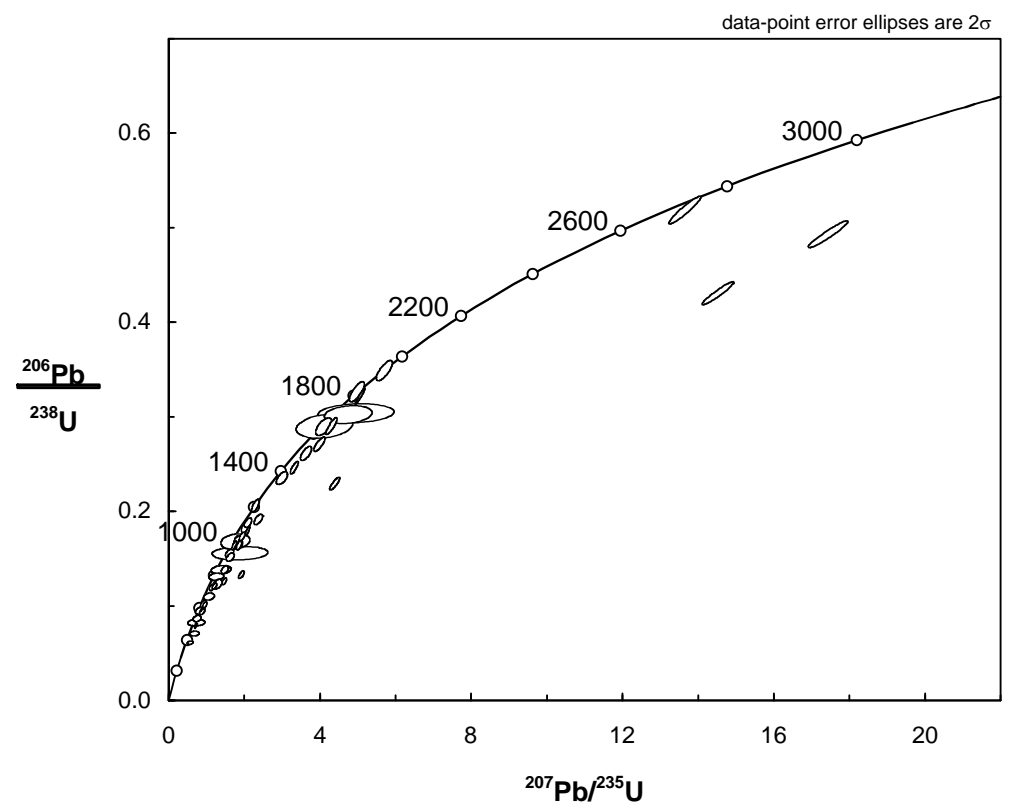

DR Fig.8b. Wetherill concordia plot for sample KU2. 


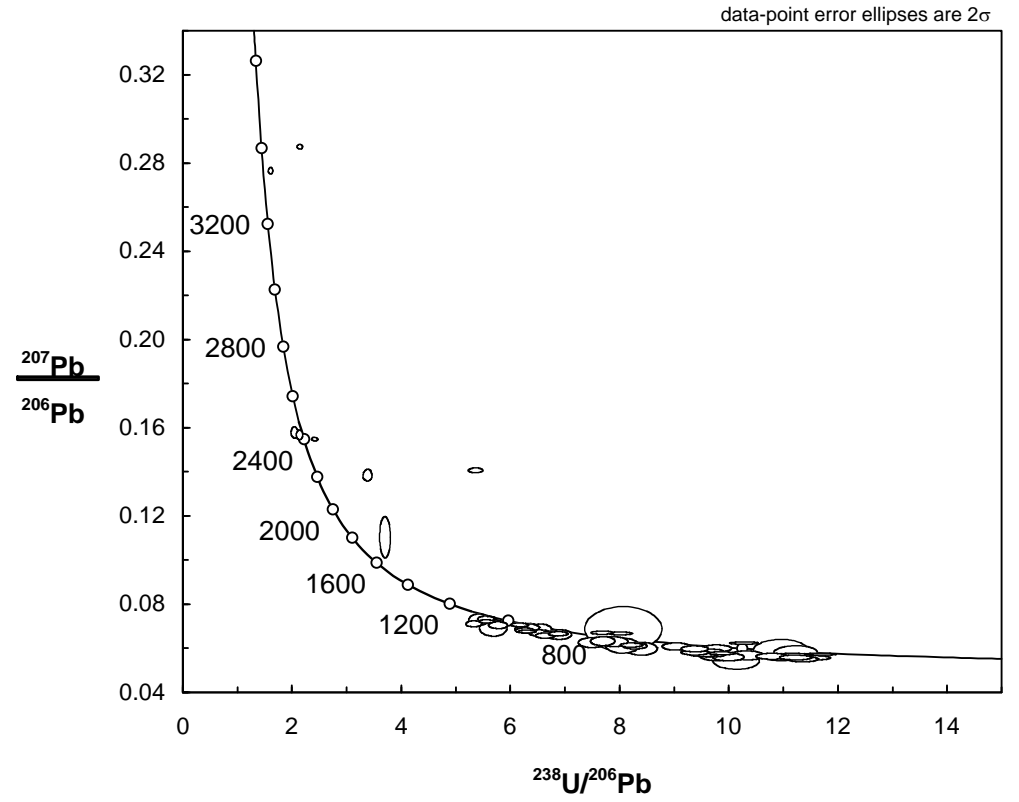

DR Fig.9a. Tera-Wasserburg concordia plot for all data from sample MBQ.

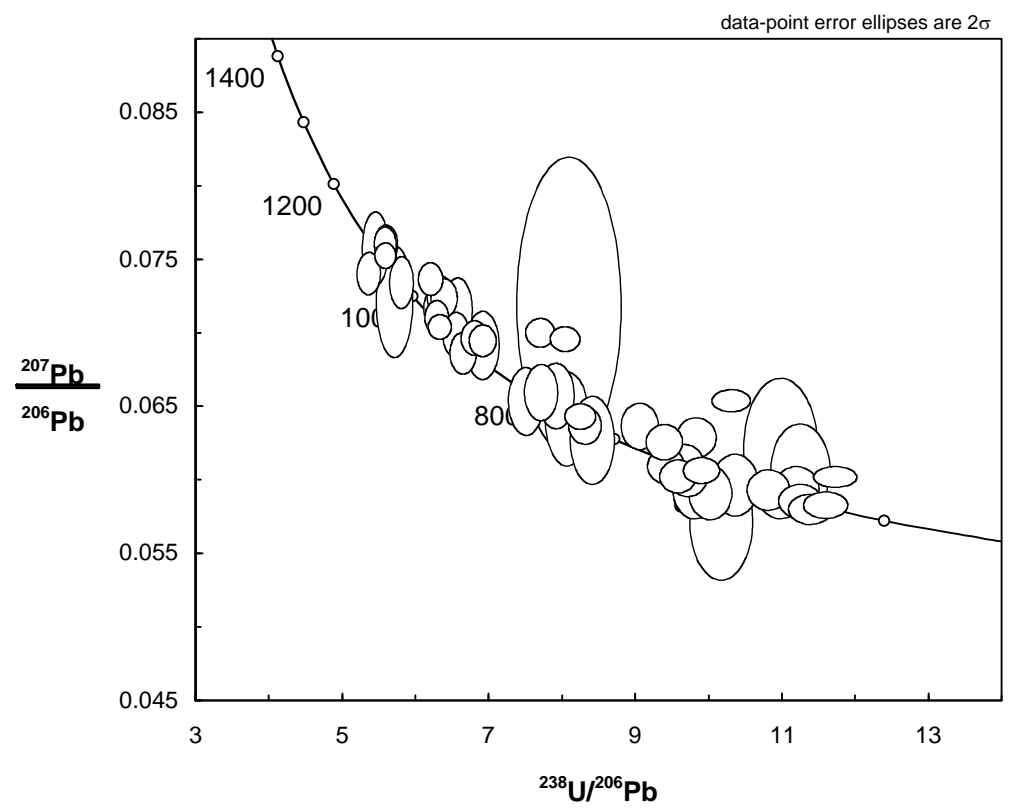

DR Fig.9b. Enlarged Tera-Wasserburg concordia plot for analyses $\leq 1100$ Ma from sample MBQ. 


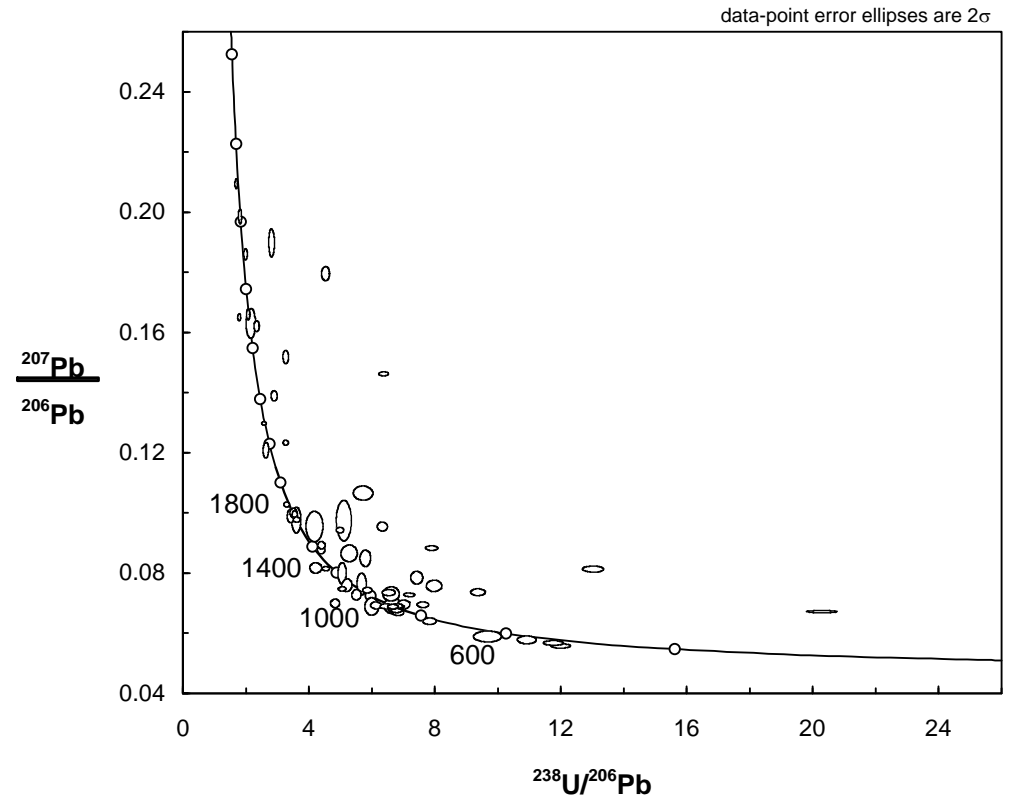

DR Fig.10a. Tera-Wasserburg concordia plot for all data from sample MS-5

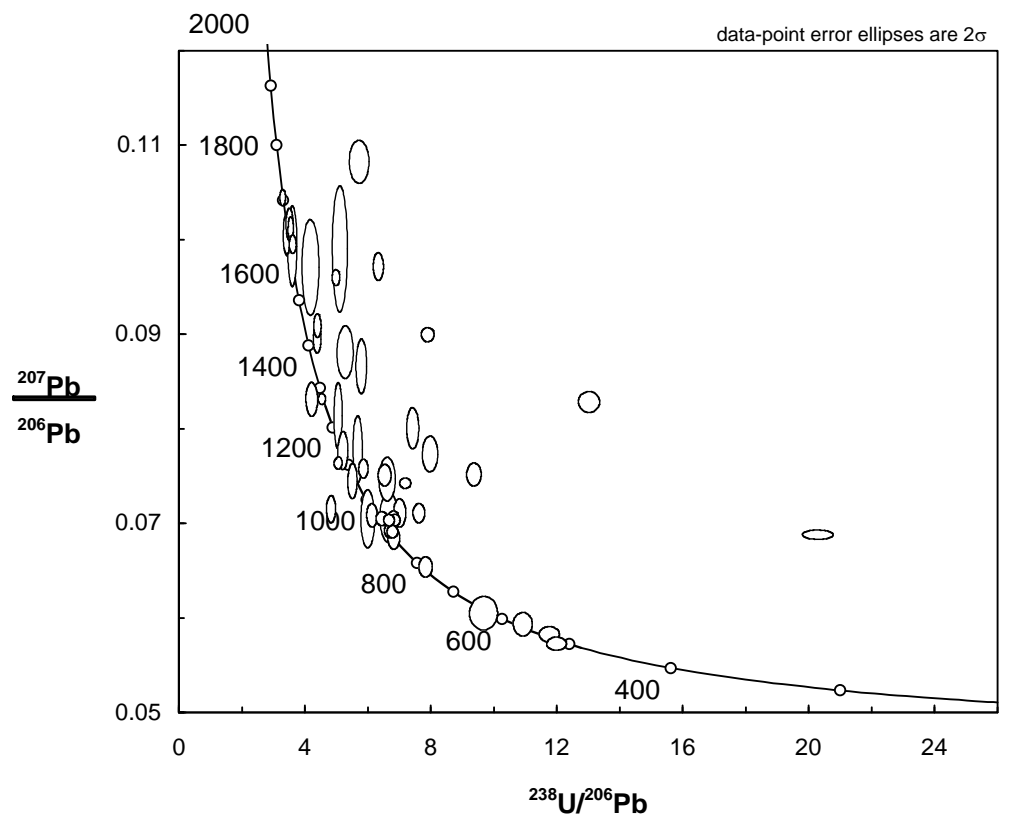

DR Fig.10b. Enlarged Tera-Wasserburg concordia plot for analyses $\leq 1900$ Ma from sample MS-5. 


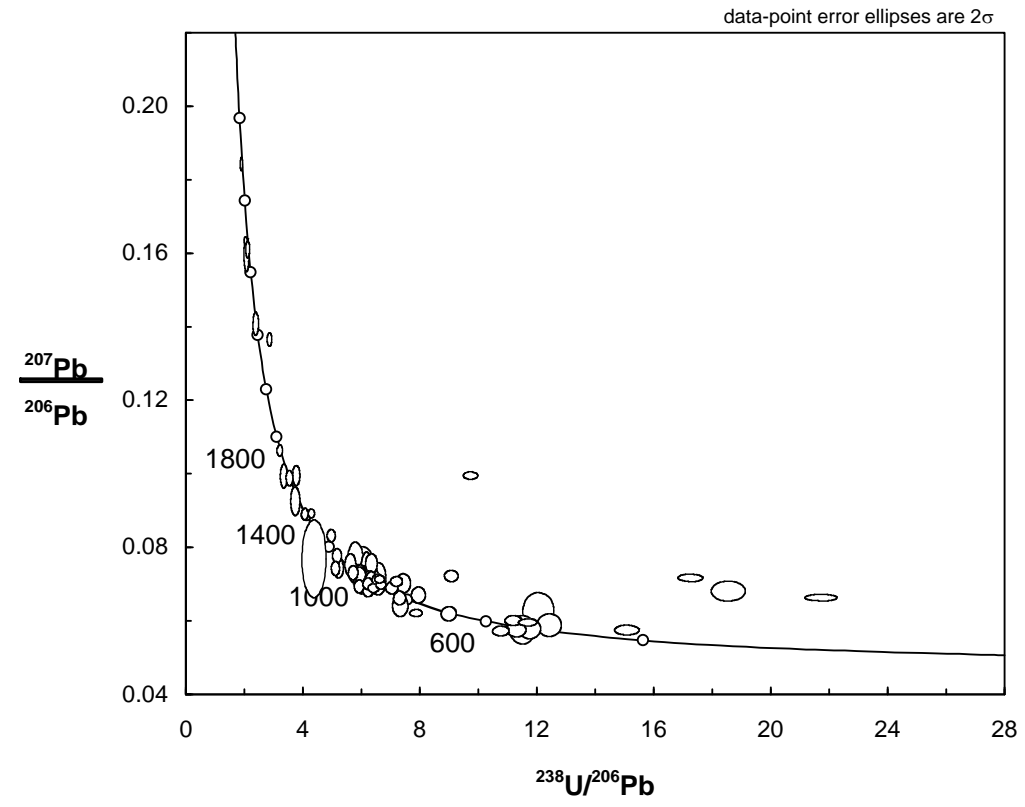

DR Fig.11a. Tera-Wasserburg concordia plot for all data from sample Thango

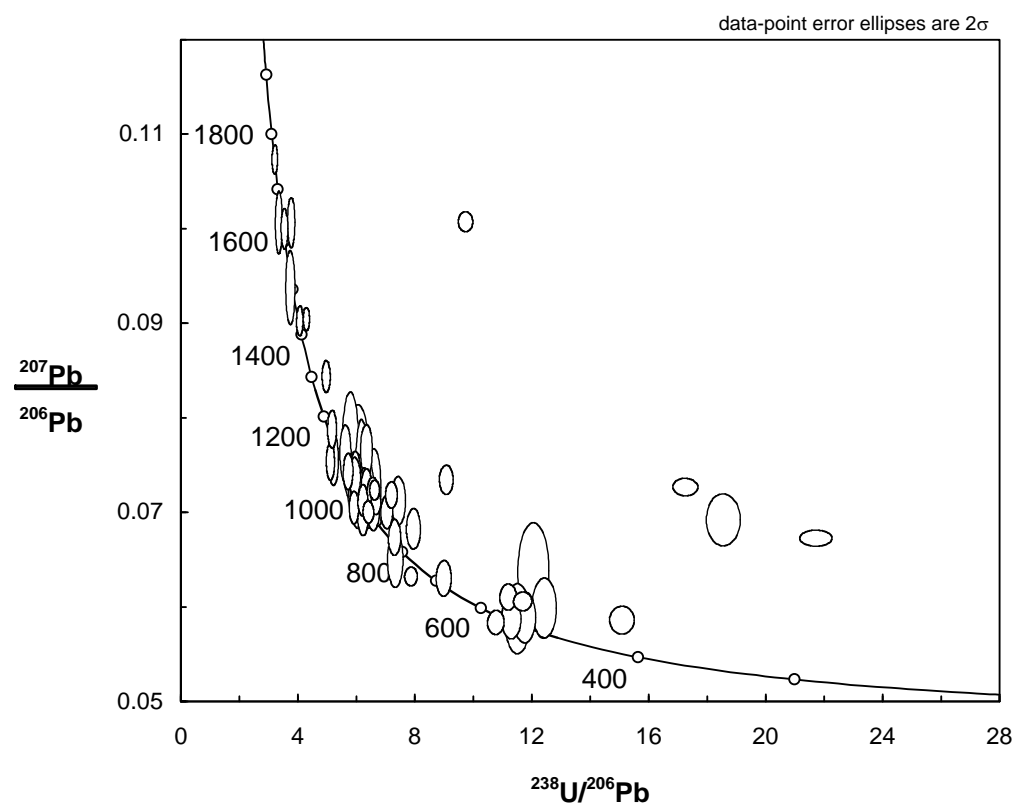

DR Fig.11b. Enlarged Tera-Wasserburg concordia plot for analyses $\leq 1900$ Ma from sample Thango. 


\section{Sample Batal}

Sixty-seven areas have been analysed on 65 zircon grains. Note that the analyses of spots 18.1 and 26.1 were not completed and so are not counted as spots/grains. Two areas were analysed each on grains 36 and 59. The analyses 18.2, 36.2 and 59.1 plot significantly above the TeraWasserburg concordia and are considered to be discordant, having lost radiogenic $\mathrm{Pb}$. Analysis 13.1 is $~ 27 \%$ discordant and 37.1 is $15 \%$ discordant. These five analyses have been excluded from the probability density plot (Fig. 3), but all are shown on the Tera-Wasserburg data repository plots.

From the 62 meaningful analyses, 5 have ages that are between 1.4-1.5 Ga. This represents $\sim 8 \%$ of that population.

\section{Sample MS-2}

Sixty-five zircon grains have been analysed. Note that the analysis of grain 29 was not completed and during the analyses of grains 15, 36, $45 \& 46$ anomalous $\mathrm{Pb}$ peak was encountered and so these analyses are flawed. The analyses of grains 30, $34 \& 37$ are significantly reversely discordant and excluded, as the normally discordant analyses from grains 31 and 49 ( 15\% \& 29\% discordant respectively). The remaining 56 analyses are close to or within uncertainty of the Tera-Wasserbrug concordia curve and used to construct the probability density plot (Fig. 3).

From the 56 meaningful analyses, 3 have ages that are between 1.4-1.5 Ga. This represents $\sim 5.4 \%$ of that population.

\section{Sample Tal}

Sixty-four zircon grains have been analysed. The grains that give the two youngest ages (37 at $\sim 405 \mathrm{Ma}$ and 43 at $\sim 680 \mathrm{Ma}$ ) plot above the Tera-Wasserburg concordia and are thus considered to be discordant, having lost radiogenic. Eight other areas analysed also yield discordant data and so have been excluded from the probability density plot; grains 1, 18, 27, 29, 44, 52, 53 \& 56. The analyses of grains 6 and 12 have not be considered meaningful as they are significantly reversely discordant.

The remaining 52 analyses are shown on the probability density plot (Fig. 3), whereas the TeraWasserburg concordia plots in the data repository show these and the discordant analyses.

Three of the grains analysed have ages in the range $1.4-1.5$ Ga representing $\sim 5.8 \%$ of the meaningful data population.

\section{Sample PV}


Sixty-five zircon grains have been analysed. A number of grains are enriched in common $\mathrm{Pb}$ as can be seen on the Tera-Wasserburg concordia and on the ${ }^{204} \mathrm{~Pb}$ corrected Wetherill plot these are seen to be discordant. The analysis of grain 5 yielded the youngest age, but it is discordant. Other discordant analyses are grains 3, 6, 21, 24, 26, 34, 35, 44 and 61

The remaining 55 analyses are shown on the probability density plot (Fig. 3), whereas the TeraWasserburg concordia plots in the data repository show these and the discordant analyses.

Two of the grains analysed have ages in the range 1.4-1.5 Ga representing $~ 3.6 \%$ of the meaningful data population.

\section{Sample Tal-GKM}

Sixty six zircon grains have been analysed. Note that there is no grain 31 analysis. The analysis of grain 20 gives a very young date, but the analysis plots well above the Tera-Wasserburg concordia curve and so is interpreted to be discordant, having lost significant amounts of radiogenic $\mathrm{Pb}$. The analysis of grain 8 is 32\% discordant. The remaining 64 analyses are shown on the probability density plot (Fig. 3), and the Tera-Wasserburg concordia plots in the data repository show these and the discordant analyses.

There are no grains with ages in the range 1.4-1.5 Ga. It is notable that there are a significant number of grains with ages in the range 750-900 Ma, perhaps reflecting a source from the east Sahara region of west Gondwana.

\section{Sample NY-11}

Sixty zircon grains have been analysed. The Tera-Wasserburg plot shows a significant number of analyses above the concordia curve. However, as can be seen from a Wetherill plot of the

${ }^{204} \mathrm{~Pb}$ corrected data (see DR), the analyses are dominantly within uncertainty of the concordia curve, i.e., concordant. Thus the measured ${ }^{204} \mathrm{~Pb}$ can account for the elevated, total ${ }^{207} \mathrm{~Pb} /{ }^{206} \mathrm{~Pb}$ ratios for some analyses as seen on the T-W plot; it is normal common $\mathrm{Pb}$ and can be accounted for by the measured ${ }^{206} \mathrm{~Pb} /{ }^{204} \mathrm{~Pb}$ ratio. Most significantly, this means that the two analyses at 525 Ma have geological significance, as do the other between 525 and 575 Ma.

All 60 analyses are shown on the probability density plot (Fig. 3), and both the Tera-Wasserburg plot and the Wetherill plot in the data repository show these and the discordant analyses.

Four grains have ages in the range $1.4-1.5$ Ga representing $~ 6.7 \%$ of the population.

\section{Sample WL-270}

Sixty zircon grains have been analysed. The Tera-Wasserburg plot shows a some analyses above the concordia curve, however, as can be seen from a Wetherill plot of the ${ }^{204} \mathrm{~Pb}$ corrected data 
(see DR), most of this is accounted for by the measured common Pb. Despite this, on the TeraWasserburg concordia diagram the analysis of grain 10 plots well above a group of 6 analyses that have ${ }^{206} \mathrm{~Pb} /{ }^{238} \mathrm{U}$ ages between $482 \pm 6 \mathrm{Ma}$ and $507 \pm 5 \mathrm{Ma}$. The $474 \pm 12 \mathrm{Ma}$ date for grain 10 is interpreted to be of an area of zircon that has lost radiogenic Pb. For the other 6 grains, there is no single age grouping, but the probability density plot and mixture modelling (Sambridge \& Compston, 1994) suggest that there are groupings at about $485 \mathrm{Ma}$ and $504 \mathrm{Ma}$.

The analyses of grains 4, 16, 29, 43, 49, 51 and 58 are discordant, and so they are not included in the probability density plot (Fig. 3). The remaining 53 analyses are shown on the probability density plot Fig. . All 60 analyses are shown on the the Tera-Wasserburg plots in the data repository show these and the discordant analyses.

Three grains have ages in the range 1.4-1.5 Ga representing 5\% of the population.

\section{Sample KU2}

Only 50 zircon grains have been analysed from this sample, as unfortunately many grains are metamict and not in a good state for U-Pb analyses. The analyses of grains 27 and 31 yield young dates that are not used in the probability density plot as the areas analysed are interpreted as having lost radiogenic $\mathrm{Pb}$. A further 11 other grains are excluded also on the basis of being discordant; grains 10, 12, 17, 24, 25, 37, 41, 44, 45, 46 \& 47. The remaining 37 analyses are shown on the probability density plot (Fig. 3). However the data repository shows TeraWasserburg plots for these and the discordant analyses.

One grain is in the age range $1.4-1.5 \mathrm{Ga}$, or $2.7 \%$ of this somewhat limited dataset.

\section{Sample MBQ}

Fifty-nine zircon grains have been analysed from this sample; there was no completed analysis for grain 10. The analysis of grain 36 is extremely rich in common $\mathrm{Pb}$ and so it is not plotted on the Terra-Wasserbrug concordia (see data repository). The remaining analyses plot close to, or within uncertainty of, the T-W concordia, although a number are interpreted to have lost radiogenic Pb; namely grains 16, 22, 35 and 52.

The remaining 54 analyses are shown on the probability density plot (Fig. 3), whereas the data repository shows Tera-Wasserburg plots for the majority of the analyses.

There are no grains in the age range 1.4-1.5 Ga.

\section{Sample MS-5}

Sixty-six zircon grains have been analysed from this sample; there was no completed analyses of grains 54 or 55 (not included in the 66 total). As is clearly evident on the Tera-Wasserburg plot, 
many of the areas analysed are enriched in common $\mathrm{Pb}$ and from the 204Pb corrected data these are seen to be discordant. With respect to the 3 youngest analyses as recorded by grains (62, 64 \& 66), they plot well above the T-W concordia and interpreted to be discordant, having lost radiogenic $\mathrm{Pb}$. There are another 18 grains analysed that yield discordant data.

The remaining 45 analyses are shown on the probability density plot (Fig. 3). The T-W plots for the data are given in the data repository.

Two grains have ages in the range 1.4-1.5 Ga, representing 4.3\% of the final meaningful dataset.

\section{Sample Thango}

Sixty-four zircon grains have been analysed from this sample. As is evident on the TeraWasserburg plot, the majority of the areas analysed plot close to the $\mathrm{T}-\mathrm{W}$ concordia although there are number of analyses at the younger end that are significantly enriched in common $\mathrm{Pb}$. The analyses of grains 18, 32, 36 and 55 are interpreted to be discordant, having lost radiogenic $\mathrm{Pb}$. There are another 3 grains analysed that yield discordant data: 5, 43 and 53.

The remaining 57 analyses are shown on the probability density plot (Fig. 3). The T-W plots for the data are given in the data repository.

Three grains have ages in the range 1.4-1.5 Ga, representing $~ 5.3 \%$ of the meaningful dataset.

\section{Bibliography}

Sambridge, M.S., and Compston, W., 1994, Mixture modelling of multi-component data sets with application to ion-probe zircon ages: Earth and Planetary Science Letters, v. 128, p. 373-390. 